\title{
Optimal Reactive Power Generation for Radial Distribution Systems Using a Highly Effective Proposed Algorithm
}

\author{
Le Chi Kien $\left(\mathbb{D},{ }^{1}\right.$ Thuan Thanh Nguyen $\mathbb{D}^{2},{ }^{2}$ Bach Hoang Dinh $(\mathbb{D})^{3}$ \\ and Thang Trung Nguyen $\mathbb{D}^{3}$ \\ ${ }^{1}$ Faculty of Electrical and Electronics Engineering, Ho Chi Minh City University of Technology and Education, \\ Ho Chi Minh City 700000, Vietnam \\ ${ }^{2}$ Faculty of Electrical Engineering Technology, Industrial University of Ho Chi Minh City, Ho Chi Minh City, Vietnam \\ ${ }^{3}$ Power System Optimization Research Group, Faculty of Electrical and Electronics Engineering, Ton Duc Thang University, \\ Ho Chi Minh City 700000, Vietnam \\ Correspondence should be addressed to Bach Hoang Dinh; dinhhoangbach@tdtu.edu.vn
}

Received 14 July 2020; Revised 15 October 2020; Accepted 21 January 2021; Published 2 February 2021

Academic Editor: Qingdu Li

Copyright (c) 2021 Le Chi Kien et al. This is an open access article distributed under the Creative Commons Attribution License, which permits unrestricted use, distribution, and reproduction in any medium, provided the original work is properly cited.

\begin{abstract}
In this paper, a proposed modified stochastic fractal search algorithm (MSFS) is applied to find the most appropriate site and size of capacitor banks for distribution systems with 33, 69, and 85 buses. Two single-objective functions are considered to be reduction of power loss and reduction of total cost of energy loss and capacitor investment while satisfying limit of capacitors, limit of conductor, and power balance of the systems. MSFS was developed by performing three new mechanisms including new diffusion mechanism and two new update mechanisms on the conventional stochastic fractal search algorithm (SFS). As a result, MSFS can reduce $0.002 \%, 0.003 \%$, and $0.18 \%$ of the total power loss from SFS for the three study systems. As compared to other methods, MSFS can reduce power loss from $0.07 \%$ to $3.98 \%$ for the first system, from $3.7 \%$ to $7.3 \%$ for the second system, and from $0.92 \%$ to $6.98 \%$ for the third system. For the reduction of total cost, the improvement level of the proposed method over SFS and two other methods is more significant. It is $0.03 \%, 1.22 \%$, and $5.76 \%$ for the second system and $2.31 \%, 0.87 \%$, and $3.77 \%$ for the third system. It is emphasized that the proposed method can find the global optimal solutions for all study cases while SFS was still implementing search process nearby or far away from the solutions. Furthermore, MSFS can converge to the best solutions much faster than these compared methods. Consequently, it can be concluded that the proposed method is very effective for finding the best location and size of added capacitors in distribution power systems.
\end{abstract}

\section{Introduction}

Electric distribution networks have a very important role in receiving electricity from transmission power network and supplying the electricity to loads. The main difference between the distribution networks and transmission networks is voltage level, leading to another difference, which is total active power loss due to the impact of resistance of conductors. The active power loss is dependent on the result of $R I^{2}$ [1] (where $R$ is the resistance of conductor and $I$ is current flowing the conductor). Current value is a main factor to result in a high active power loss in distribution networks while $R$ is a constant in the networks. The smaller the voltage is, the higher the active power loss is. Hence, active power loss is a significant issue when distribution networks are working for supplying power energy to loads.

In order to reduce the high active power loss in distribution networks, experts have proposed two basic methods including network reconfiguration $[2,3]$ and shunt capacitor installation $[4,5]$. The network reconfiguration method is to change status of switches, either open or close to change direction of current. Basically, distribution networks are supplied at one point, which is called slack node, and it is obvious that all loads in the networks are being supplied by the slack node via distribution lines. Thus, the method cannot reduce power supplied by the slack node and 
just mainly reduces high current in lines with high resistance or long length and increases lower current in other lines. By using the method, power loss is effectively reduced. In addition, voltage profile is also improved, but the improvement is not certain or insignificant. On the contrary, the second method using shunt capacitors can reduce reactive power that is supplied by the distribution lines. Loads can consume reactive power from both the added capacitors and the distribution lines or only consume reactive power from the added capacitors. As a result, current in distribution lines can be reduced considerably and power factor is increased effectively. In addition, another benefit from the capacitor installation is the reduction of voltage drop in the line. In fact, as current is smaller, the voltage drop is decreased accordingly. In addition to the two basic methods, other methods can be applied such as (1) placement of distributed generators $[6,7],(2)$ the combination of reconfiguration and capacitor placement [8,9], (3) the combination of reconfiguration and distributed generator placement $[10,11]$, and (4) the combination of capacitor placement and distributed generator placement $[12,13]$. In this paper, we focus on the second basic method of optimally installing capacitors with the task of determining the best location and the best rated reactive power. The best location and the best rated power of these added capacitors are for reaching two single-objective functions in which the first objective function is to minimize the total active power loss on all distribution lines [14-45] and the second objective function is to minimize the total cost of energy loss and capacitor investment [34, 46-49]. In addition, operation limit of conductor and operating voltage of loads are always supervised seriously via the consideration of maximum current of lines [50] and the consideration of upper and lower voltage [51]. The problem of capacitor placement has attracted a huge number of researchers in proposing optimization tools and capacitor placement strategies based on configuration and practical analysis. Approximately all the applied methods are different; however, the common study of all the methods is the active power loss reduction. In [1], a proposed method with two stages was applied for two systems with 15 and 33 nodes. In the first stage, a sequence of compensated nodes is first determined by using an iterative algorithm with the placement of one capacitor for minimizing power loss. Then, the optimal size of capacitors at the determined nodes was found by minimizing a loss saving equation, which is a function of capacitors' current. This method could reach lower power loss than original networks without capacitor placement. However, the method had to suffer from the limits of application for large-scale problem with a high number of load nodes because each capacitor is tried to be placed at all nodes excluding slack node in the first stage. So, it will be time consuming for trying one by one node in a large-scale system with too many nodes. For example, it must try fourteen times for 15-node network and 32 times for 33-node network. Thus, the higher the number of nodes is, the longer the simulation time is. The method can solve the high power loss issue, but it is not a good choice for the radial distribution networks because there was no comparison between the method and other ones in the study. Another similar method was proposed in [4] for maximizing saving energy loss as compared to original radial distribution networks. The study replicated the first stage of determining compensated nodes where reactive power is necessary to reduce current flowing in distribution lines. Then, the second stage is to determine the most appropriate size for each shunt capacitor by maximizing the saving power loss compared to original network. The method can solve the problem easily and successfully, but its applications for large-scale problem also suffer from the same restriction as the two-stage method [1] because of the first stage. In fact, the method was only applied for 15-node and 33-node networks. The method was only superior to the two-stage method [1]. In 2013, another two-stage method (TSM) [14] was applied for the same problem but the application was wider and more successful thanks to the modifications on the first stage. The first stage for finding the most suitable locations is performed by using cross check fuzzy expert system and loss reduction index. So, the two-stage method could avoid the significant restriction of the methods $[1,4]$. The large-scale problem with 69 nodes was successfully solved and the method could reach better loss reduction than other previous methods; however, the simulation time was still the major disadvantage of the method. In [15], the two-stage method proposed in [4] was applied to determine distributed generator location and size in the radial distribution network. The method could find location and size of the distributed generator successfully and effectively as the obtained power loss was less than that in capacitor location and size determination problem. However, the method one more time shows its disadvantage since the most complicated study case was 33-node network. Clearly, the two-stage methods could not reach the highest performance for the problem of determining location and size of capacitors and distributed generators. Due to major disadvantages such as not applicable for large-scale network and time consuming, the two-stage methods were not applied widely and they must be replaced with more potential metaheuristics such as genetic algorithm variants, particle swarm optimization (PSO) variants, and other recent ones. PSO based on inertia weight and constriction factor (IWC-PSO) was applied for finding reactive power generation of capacitors while the loss sensitivity factor method was proposed to determine candidate nodes, where capacitor placement is necessary [16]. The loss sensitivity factor method was used to identify lowvoltage nodes or capacitor location that can improve voltage of other low-voltage nodes, where capacitors are not installed. Single and multiple capacitors were installed in five distribution networks with 10, 15, 34, 69, and 85 nodes, and voltage profile was significantly improved as compared to voltage profile in original networks and results from [1]. It should be noted that objective function of the study [1] was loss reduction, whereas that in [16] was voltage enhancement. So, the comparison between the two-stage method [1] and IWC-PSO [16] was not suitable. The IWC-PSO continued to be applied for the problem with two single-objective functions including power loss and voltage profile [17]. The fuzzy method was used to identify candidate nodes, and then the PSO method determined the most suitable size 
for capacitors at the nodes. The largest study case was the 69node network, and results were compared to original networks. Another study [18] also applied the fuzzy method to find the most suitable locations to place capacitors, and then multiagent particle swarm optimization (MAPSO) was proposed to determine the size of capacitors. MAPSO was demonstrated to be superior to only the conventional PSO for the 69-node radial distribution network with the consideration of active and reactive power losses and voltage profile. A set of different PSO methods with different distributions (including Gaussian, Cauchy, and chaotic distributions) and different equations for calculating velocity (including weight inertia factor and constriction factor) was applied for identifying location and size of capacitors [19]. The study is different from other applications of PSO methods above since capacitors' location was selected to be control variables. Due to the selection of control variables, the study [19] could skip the first stage of determining location of capacitors by using loss reduction index as the studies $[1,4]$, loss sensitivity factor as the study [16], and fuzzy method as the study $[17,18]$. There were fifteen PSO methods to be applied for determining the best one for the problem of finding both location and size of capacitors. The comparisons from two study cases in the 9-feeder radial distribution network showed that the PSO method with uniform distribution and chaotic distribution was the best one for the smallest power loss. In addition to the comparison among the PSO methods, the best PSO method was compared to genetic algorithm (GA) and tabu search algorithm (TSA). In general, the PSO method was the best one among fifteen PSO methods and superior to two other lowly effective methods such as GA and TSA for only a small-scale system with 9 feeders and 10 nodes. Hence, the real performance of this method was still a question for the problem. Different GA variants including conventional GA [20-23], micro GA (MGA) [24], real coded genetic algorithm (RCGA) [25], and the combination of fuzzy and GA (FGA) [26] were the applied solution methods to optimally place capacitors in the radial distribution networks. The applications of conventional GA did not demonstrate the high performance of GA because the study cases were simple and comparisons were mainly between the original networks and networks with capacitor placement. In fact, Taiwan network and Iran network were, respectively, studied in [20,23] while 23-node network and 33-node network were, respectively, studied in [21,22]. These studies were poor in comparisons and study cases. In [24], MGA was applied for Italian network and compared with GA for comparison. In [25], capacitors were installed in three networks with 15,34 , and 69 nodes by using RCGA. The power loss reduction of the cases with and without capacitor placement was compared. Clearly, all the studies have the same shortcoming of poor study cases and comparisons. In addition to the application of GA for single-objective problem with only power loss reduction, a multiple-objective problem with voltage profile improvement and total cost reduction was solved by the implementation of GA for getting a set of solutions. Then, the fuzzy method was employed to determine the most appropriate compromise solution. The paper only executed the comparison of networks with and without capacitor placement rather than showing the real performance of GA as compared to other methods. So, GA was not a real effective method for the problem [27].

In addition to these method groups, other smaller groups were also applied for the same problem of capacitor placement such as mixed integer nonlinear programming-based method (MINPM) [27], gravitational search algorithm (GSA) [28], the combination of GSA and weight inertia factor-based PSO (WIFPSO-GSA) [29], bacterial foraging optimization algorithm (BFOA) [30, 31], flower pollination algorithm (FPA) [32,33], teaching-learning algorithm (TLA) [34], whale optimization algorithm (WOA) [35], power loss index-based improved harmony algorithm (PLI-IHA) [36], cuckoo search algorithm (CSA) [37], improved mutation technique-based differential evolution (IMT-DE) [38], moth swarm algorithm (MSA) [39], ant colony algorithm based on loss sensitivity factor (LSF-ACA) [40], heuristic method based on network configuration (NCB-HM) [41, 42], combined practical method (CPM) [43, 44], hybrid method (HM) [45], direct search optimization algorithm (DSOA) [46], penalty free method-based heuristic algorithm (PFHA) [47], inclusion and variable interchange algorithm (IVIA) [48], water cycle algorithm [49], and grey wolf algorithm (GWA) [49]. Among the methods, MINPM, NCB-HM, CPM, IVIA, and DSOA are not metaheuristic algorithms based on population and they are mainly dependent on the real configuration of networks. So, the application of the methods is not performed for arbitrary systems without the analysis on the power loss and voltage drop. Other metaheuristic algorithms can reach better results than PSO and GA method groups; however, the real performance of these methods was not demonstrated clearly. In fact, these methods have been run by setting different values to population and iterations without comparisons. It is noted that metaheuristic algorithms can result in good solutions if they spend high computation time due to high value of population and iterations. In terms of considered objective functions, almost all previous studies focused on the purpose of reducing power loss of all branches and neglecting the total compensation capacity. Observing the results from BFOA [30, 31], FPA [33], NCB-HM [42], CPM [43, 44], and HM [45], it could be seen that only the obtained power loss was compared, methods with smaller power loss were concluded to be more effective, and total compensation of all capacitors was not discussed. For some cases, methods with higher capacity could reach less power loss, but for other cases, lowperformance methods even with higher compensation capacity still obtained higher power loss. The shortcoming has been pointed out, and it was noted that higher compensation capacity will use higher capacitor investment purchase cost [46]. For tackling the issue, power loss and compensation capacity were converted into cost by calculating energy loss cost and considering capacitor purchase cost. The sum of energy loss cost and capacitor purchase cost was then considered as an objective for performance comparison.

In summary, the previous studies have two main shortcomings in which the former is not to further investigate the convergence speed of compared methods and the latter is not to consider compensation capacity. In this paper, 
the two shortcomings are solved by collecting population, iteration, and the simulation time in addition to the consideration of sum of energy loss cost and capacitor purchase cost as the second single-objective function. A proposed method, called modified stochastic fractal search optimization algorithm (MSFS), is applied to reach two single-objective functions including total power loss and the total cost of loss and capacitor of distribution systems. The performance of the proposed method is investigated by comparing total loss, total cost, and convergence speed. Furthermore, the proposed method is also demonstrated to be more effective than its original method, stochastic fractal search optimization algorithm (SFS). SFS was also an effective optimization tool developed in 2015 for reaching results from a 23function set [52]. The method could attract the concern from a huge number of researchers thanks to its superiority to eight popular and effective algorithms such as PSO, differential evolution algorithm (DE), gravitational search optimization algorithm (GSOA), artificial bee colony optimization algorithm (ABCOA), cuckoo search optimization algorithm (CSOA), improved version of CSOA (ICSOA), animal migration optimization algorithm (AMOA), and backtracking search optimization algorithm (BSOA). SFS was applied and reached promising results for different optimization problems in electrical engineering such as distributed generator placement in distribution systems [53], environmental and economic dispatch [54], PID controller design [55], economic load dispatch [56], and reconfiguration of distribution networks [11]. SFS is characterized by three mechanisms of generating new candidate solutions including diffusion mechanism and two update mechanisms. The diffusion mechanism uses Gaussian distribution mean while the two update mechanisms use mutation of DE with modifications. The original structure of SFS was considered to be ineffective for complicated problems, and a number of improved versions were suggested for different problems such as multiple area economic load dispatch [57], optimal dispatch of reactive power [58], photovoltaic system parameter estimation [59], optimal transmission network power flow [60], and optimal generation control of interconnected power systems [61]. In the proposed MSFS method, we use modifications on the three mechanism to improve the search performance. In the diffusion mechanism, we employ another new formula and keep one out of two old formulas of SFS to balance the global search and local search. In the two update mechanisms, a new algorithm is proposed for the two update mechanisms and only performed for the first half of the whole population with better quality. The new algorithm can balance the global and local search effectively while the number of fitness evaluations is decreased to half of SFS. Each proposed modification and all the proposed modifications are tested on three radial distribution networks (RDNs) including 33, 69, and 85 nodes with two different single-objective functions such as reduction of power loss and reduction of the sum of energy loss cost and capacitor investment cost. As a result, the core contributions of paper are summarized as follows:

(i) Core shortcomings of SFS are found.

(ii) New formula and new algorithms are proposed for tackling shortcomings of SFS.

(iii) Each proposed modification and the whole modifications are tested and proved

(iv) The proposed MSFS is superior to its conventional method in terms of using smaller number of computation iterations, spending shorter simulation time, and reaching better results.

In terms of the organization of the paper, the remaining parts of the paper are as follows. Two single-objective functions and considered constraints are presented in detail in Section 2. The original SFS method and the proposed MSFS method are clearly explained in Section 3. Section 4 explains the implementation of MSFS for the problem. In Section 5, three systems with two different objective functions are employed to run SFS and MSFS for comparison and discussion. Finally, the summary and conclusions are presented in Section 6.

\section{Problem Formulation}

2.1. Objective Functions. The conventional problem of adding capacitors in the distribution system aimed to reduce the active power loss [14] as much as possible while the total compensation capacity was not considered as long as the total compensation capacity was not higher than reactive power demand of loads. However, some studies have pointed out the major issue of the problem was the cost of buying capacitors and energy loss. So, energy loss cost and capacitor investment cost were considered as an objective. In this paper, we consider two single-objective functions in which the first objective is to minimize the total active power loss (TPL) while the second objective is to minimize the sum of energy loss cost and capacitor investment cost. The two objectives are presented as follows.

2.1.1. TPL Reduction. Distribution network is comprised of a high number of distribution lines, and total active power loss on the lines is significant as a result. Consequently, capacitors are added at suitable nodes to reduce current of lines and reduce the total active power loss on all lines. The first objective of the problem is to minimize the total active power loss as shown in the following formula [30]:

$$
\text { minimize TPL }=\sum_{i=1}^{\mathrm{Nbr}} 3 I_{i}^{2} R_{i}
$$

where $I_{i}$ is the current magnitude in the $i$ th branch and $R_{i}$ is the $i$ th branch resistance. 
2.1.2. Energy Loss and Investment Cost Reduction. When placing capacitors in distribution networks, power loss and energy loss can be reduced or even highly reduced if the compensation capacity is very high. The high compensation capacity leads to a high cost for capacitor investment, and the energy loss reduction cannot compensate the high cost of capacitor investment. As the sum of energy loss cost and the investment cost is low, the capacitor placement is effective. Thus, the energy loss cost and the capacitor investment cost are the main factors of the second objective, and the objective is mathematically formed as follows [46]:

$$
\text { minimize } \mathrm{TC}=\operatorname{Pr}_{e} \sum_{m=1}^{M} \mathrm{TPL}_{m} \cdot T_{m}+\operatorname{Pr}_{c} \sum_{j=1}^{\mathrm{Nc}} Q_{c j} \text {, }
$$

where TC is the total cost of energy loss cost and investment cost; $\mathrm{TPL}_{m}$ is the total active power loss of the $m$ th load level; $\operatorname{Pr}_{e}$ and $\operatorname{Pr}_{c}$ are the price of each $\mathrm{kWh}$ and each $\mathrm{kVAr} ; T_{m}$ is the number of hours for the $m$ th load level; and $M$ is the number of load levels.

2.1.3. Constraints. The distribution power network can work stably and effectively if voltage magnitude of all nodes is within working range and current of branches is not higher than the maximum limit of conductor. In addition, the total reactive power of all capacitors must not be higher than the total reactive power of all loads. The whole constraints are as follows:

(i) Voltage limit constraint: voltage of each node in the system must be within the working range of the network. The working range is the same for all nodes while the voltage magnitude of nodes can be different. The constraint is mathematically formulated as follows [34]:

$$
U_{\min } \leq U_{i} \leq U_{\max } ; \quad i=1, \ldots, \mathrm{Nb},
$$

where $U_{\min }$ and $U_{\max }$ are the minimum and maximum working voltage of all nodes in the radial distribution networks and $U_{i}$ is the voltage of the $i$ th node.

The constraint plays a very important role in stabilizing the operation of loads and the whole distribution system as well as achieving the high benefit for both customers and power companies. In case that voltage of loads is smaller than $U_{\text {min }}$, load cannot work at the rated power and loads can be damaged if the voltage of loads is higher than $U_{\text {max }}$.

Limit constraint of total capacity of capacitors: capacitors are very useful in improving voltage profile and reducing power loss of the distribution networks. However, as the size of capacitors is high enough, the power loss becomes higher, whereas voltage profile is always improved and even voltage of loads can be equal to rated value or higher than rated value. Consequently, the total compensated reactive power of capacitors must be limited as the following constraints [30]:

$$
\sum_{j=1}^{\mathrm{Nc}} Q_{c j} \leq Q_{c, \max }
$$

where $Q_{c j}$ is the reactive power generation of the $j$ th capacitor and $Q_{c, \text { max }}$ is the maximum reactive power generated by all capacitors.

As demonstrated in [62], the maximum compensated power of capacitors could not be higher than the total reactive power of all loads because the redundant reactive power will continue to flow into other nodes where reactive power is necessary. The active power flows will cause power loss unintentionally. So, the compensation capacity must satisfy the constraint below [43]:

$$
Q_{c, \max } \leq \sum_{i=1}^{\mathrm{Nb}} Q_{i},
$$

where $Q_{i}$ is the $i$ th load reactive power.

Limit constraint of branch current: one main parameter of each distribution line is the maximum current magnitude that conductor can work stably in operating time. So, active and reactive power flows that are distributed in all branches should be appropriate and not higher than the capacity of distribution lines. In order to satisfy the requirement, active and reactive power flows are converted into current magnitude and the current magnitude must not be higher than the maximum current of conductor as shown in the model below:

$$
I_{i} \leq I_{i}^{\max } ; \quad i=1, \ldots, \mathrm{Nbr},
$$

where $I_{i}^{\max }$ is the $i$ th branch capacity.

\section{The Proposed Method}

3.1. Classical Stochastic Fractal Search Optimization Algorithm (SFS). SFS performs three different techniques for updating new solutions in each iteration. Among the three techniques, the first one, called diffusion technique, can produce many new solutions for each old solution dependent on the number of diffusions, $N_{\mathrm{Df}}$. Each old solution can be newly updated $N_{\mathrm{Df}}$ times, and there will be $\left(N_{\mathrm{Df}} \times N_{\mathrm{Po}}\right)$ new solutions in the first technique as a result of the diffusion effect. On the contrary, the two remaining techniques, called the first update technique and the second updated technique, produce much less new solutions than the first technique. The first and second update techniques perform update at most a number of solutions equaling population. So, the total newly updated solutions over the whole search of SFS are $\left(N_{\mathrm{Df}} \times N_{\mathrm{Po}}+2 \times N_{\mathrm{Po}}\right) \times N_{\text {Iter }}$. The three techniques of the method are described in detail as follows.

3.1.1. Diffusion Technique. Diffusion technique of SFS can diversify the search process by exploiting search space around each current solution and the best solution. Each 
solution produces a random number (called $\mathrm{rd}_{s}$ ) within zero and one, and this random number is compared to a factor, called diffusion factor (DF). The technique can be formulated by

$$
X_{s}^{\text {new }}= \begin{cases}\text { normrnd }(\text { Gbest, sigma })+\varepsilon \times\left(\text { Gbest }-X_{s}\right), & \text { if } \operatorname{rd}_{s}<\mathrm{DF}, \\ \text { normrnd }\left(X_{s}, \text { sigma }\right), & \text { otherwise }\end{cases}
$$

In the equation, normrnd (Gbest, sigma) produces random numbers around Gbest with a standard deviation of sigma, which is obtained by

$$
\text { sigma }=\mid \frac{\log \left(C_{\text {Iter }}\right)}{C_{\text {Iter }}} \times\left(X_{s}-\text { Gbest }\right) \mid
$$

3.1.2. The First Update Technique. In the first update technique, SFS can update the whole population at most, but normally the number of newly updated solutions is less than the population size. In the first step, the whole population must be sorted based on the fitness function. Better solutions with smaller fitness function are placed at the end of the population, whereas worse solutions with higher fitness function are put at the top of the population. The order number of each solution after sorting is stored and set to $R_{s}$. Each solution is evaluated by calculating a quality index as the following equation:

$$
\mathrm{QI}_{s}=\frac{R_{s}}{N_{\mathrm{Po}}} .
$$

The quality index of each solution cannot be lower than 0 and higher than 1 . The best solution with the last position will get the highest index with $\mathrm{QI}_{s}=1$, and the worst solution with the first position will get the lowest index with $\mathrm{QI}_{s}=1$ / $N_{\text {Po. }}$

In the second step, each solution will produce a random number $\mathrm{rd}_{s}$ within zero and 1 . If $\mathrm{rd}_{s}$ is equal to or higher than $\mathrm{QI}_{s}$, the considered solution will be updated by the following formula:

$$
X_{s}^{\text {new }}=X_{1}-\varepsilon \times\left(X_{2}-X_{s}\right)
$$

For another case (i.e., $\operatorname{rd}_{s}$ is less than $\mathrm{QI}_{s}$ ), the sth solution remains unchanged. Clearly, solutions with high quality do not have a high possibility to be newly updated.

3.1.3. The Second Update Technique. In the second update technique, all solutions have the same update possibility, but the methods for updating the solution can be different. Each solution also produces a random number $\mathrm{rd}_{s}$, and this value is compared to 0.5 for determining which method is employed for the considered solution. The second update technique is formulated as follows:

$$
X_{s}^{\text {new }}= \begin{cases}X_{s}+\varepsilon \times\left(X_{3}-X_{4}\right), & \text { if } \operatorname{rd}_{s}<0.5, \\ X_{s}-\varepsilon \times\left(X_{3}-\text { Gbest }\right), & \text { else. }\end{cases}
$$

\subsection{The Proposed Modified Stochastic Fractal Search Algorithm (MSFS)}

3.2.1. Discussion on Diffusion Technique and New Diffusion Technique. The MSFS is proposed to tackle shortcomings existing in its original version. In the diffusion technique, two models in equation (7) can be used, but the new update using the second model, normrnd ( $X_{s}$, sigma), cannot be effective because the result from normrnd ( $X_{\mathrm{s}}$, sigma) is a random method without a certain base while the first model, [normrnd (Gbest, sigma $)+\varepsilon\left(\right.$ Gbest $\left.-X_{s}\right)$ ], is much more effective by using the best solution and a step size based on a certain condition using $\left[\varepsilon \times\left(\right.\right.$ Gbest $\left.\left.-X_{s}\right)\right]$. Clearly, the first model uses local search by searching around the so-far best solution Gbest with a certain step size, whereas the second model uses global search by searching around all current solutions with randomization. So, the first model is suitable for problem with many near global solutions while the second model may not be effective because it is a random search without certain condition.

In the new diffusion technique, shortcoming of equation (7) can be avoided and improved by using the following equation.

$$
X_{s}^{\text {new }}= \begin{cases}\operatorname{normrnd}(\text { Gbest, sigma })+\varepsilon \times\left(\text { Gbest }-X_{s}+X_{1}-X_{2}\right)+ & \text { if } \operatorname{rd}_{s}<\mathrm{DF}, \\ \operatorname{normrnd}\left(X_{s}, \text { sigma }\right)+\varepsilon \times\left(X_{1}-X_{2}\right), & \text { otherwise }\end{cases}
$$

In equation (12), the above way can search around the best solution with large distance where the below way can exploit the search around the sth considered solution with a smaller distance. The proposed equation can support to exploit global search (i.e., nearby each considered solution) and local search (i.e., nearby the best solution) effectively. In addition, the two ways are based on certain determinations using a small step size with two solutions and a larger step size with four solutions, whereas the below way of SFS in equation (7) uses a random step size without certain condition.
3.2.2. Discussion on the Update Techniques. The first update technique shown in Section 3.1.2 has two key shortcomings including the condition for update and the method for update. The condition for update is the comparison between random number $\mathrm{rd}_{s}$ and the quality index $\mathrm{QI}_{s}$. Only solutions with $\mathrm{rd}_{s}>\mathrm{QI}_{s}$ are updated while high quality solutions with high value of $\mathrm{QI}_{s}$ do not have many chances to be updated. So, the condition limits the search around promising spaces but highly allows to exploit search space where low-quality solutions are existing. The second 
shortcoming of the technique is equation (10), which uses the subtraction, i.e., "-" from the current solution instead of addition, i.e., "+" like other methods such as PSO, bat algorithm, and cuckoo search algorithm.

In the second update technique, observing two ways shown in equation (11), we can see the major disadvantage of SFS in producing new solutions by using the second update technique. The above way has the same feature as mutation technique of differential evolution algorithm (DE), whereas the below way has two differences from the mutation technique of DE. The first difference is to use "-" to subtract a step size from the current solutions to produce new solutions, whereas the second difference is to use Gbest instead of a random solution to calculate a jumping step. As indicated in [63] for improving the performance of spider optimization algorithm and indicated in $[58,60]$ for improving the performance of SFS, the use of subtraction to decrease a step size from the current solutions could lead to worse results and slow the convergence to the global optimum solutions. The main disadvantage of SFS was shown clearly in [58] for reactive power dispatch problem and in [60] for optimal power flow problem. The two problems are complicated in terms of a high number of decision variables and complex constraints. Clearly, the two update techniques need to be improved for getting more effective result from complicated problems.

3.2.3. Modification on the Two Update Techniques. For reaching good solutions in the two update mechanisms, we propose a new technique for producing new solutions. In the first update, only a half of population with better quality is newly updated. Then, the new solutions are evaluated and compared to old solution to keep better one and abandon worse ones. The whole population is sorted to arrange good solutions with lower fitness at the top of the population, and worse ones are arranged at the end of the population. As a result, the first half of population is newly updated based on the following steps:

Step 1: sort all solutions in ascending order of fitness function $\left(\mathrm{Fit}_{s}\right)$.

Step 2: calculate average fitness function of the population, Fit mean $_{\text {. }}$

Step 3: update new solutions for the first half of population based on the following equation.

$$
X_{s}^{\text {new }}= \begin{cases}X_{s}+\varepsilon \times\left(X_{1}-X_{2}+X_{3}-X_{4}\right), & \text { if Fit }_{s} \leq \text { Fit }_{\text {mean }}, \\ \text { Gbest }+\varepsilon \times\left(X_{1}-X_{2}+X_{3}-X_{4}\right), & \text { else. }\end{cases}
$$

Formula (13) shows two different ways for updating new solutions $X_{s}^{\text {new }}$ based on comparison result between the considered old solution and the mean solution of the whole population. In the first method, old solutions with better quality than the mean solution of the whole population (i.e., Fit $_{s} \leq \mathrm{Fit}_{\text {mean }}$ ) will be searched around with a distance $\left[\varepsilon\left(X_{1}-X_{2}+X_{3}-X_{4}\right)\right]$. On the contrary, old solutions with worse quality than the mean solution (i.e., $\left.\mathrm{Fit}_{s}>\mathrm{Fit}_{\text {mean }}\right)$ are abandoned and the current best solution Gbest will be searched around with a step size of $[\varepsilon$ $\left.\left(X_{1}-X_{2}+X_{3}-X_{4}\right)\right]$ in the second method. The two methods can exploit promising search zones effectively and reduce search time around ineffective zones. Furthermore, the application of the larger step with four solutions $X_{1}, X_{2}, X_{3}$ and $X_{4}$ can form a large enough jumping step to escape local zones and move fast to other global zones. The effectiveness of the proposed update techniques will be demonstrated in numerical results.

\section{The Implementation of the Proposed Method for the Considered Problem}

4.1. Initialization. In the first step of implementation, selection of control variables should be accomplished and then boundaries of the variables should be determined. In the problem, shunt capacitors are placed in order to reduce total power loss. The total loss can be reduced in maximum if the location and the capacity are the most appropriate. In a large distribution power network with a high number of nodes, the determination of the most appropriate nodes for placing capacitors is not easy if optimization algorithms are not applied. Furthermore, it is supposed to determine exact nodes to place capacitors, and the determination of capacity is also a difficulty. So, both location and capacity are control variables and must be determined by using the proposed method in the study. The location of capacitors is from node 2 to node $\mathrm{Nb}$ while the total capacity of all capacitors should not be higher than the total reactive power of loads as shown in equation (5) and demonstrated in Section 2.1. Each solution is represented as follows:

$$
X_{s}=\left[L_{j, s} Q_{c j, s}\right] ; \quad j=1, \ldots, \mathrm{Nc} \& s=1, \ldots, N_{\mathrm{Po}},
$$

where $L_{j, s}$ and $Q_{c j, s}$ are the location and size of the $j$ th capacitor corresponding to the sth solution.

Each solution $X_{s}$ is randomized satisfying the constraint below:

$$
X^{\min } \leq X_{s} \leq X^{\max }, \quad s=1, \ldots, N_{\text {Po }},
$$

where $X^{\text {min }}$ and $X^{\text {max }}$ are called minimum solution and maximum solution and are mathematically expressed as follows:

$$
\begin{aligned}
& X^{\min }=\left[L_{j, s}^{\min }, Q_{c j, s}^{\min }\right] ; \quad j=1, \ldots, \text { Nc \& } s=1, \ldots, N_{\mathrm{Po}}, \\
& X^{\max }=\left[L_{j, s}^{\max }, Q_{c j, s}^{\max }\right] ; \quad j=1, \ldots, \mathrm{Nc} \& s=1, \ldots, N_{\mathrm{Po}},
\end{aligned}
$$

where $L_{j, s}^{\min }$ and $L_{j, s}^{\max }$ are the minimum location and the maximum location in the considered distribution network and $Q_{c j, s}^{\min }$ and $Q_{c j, s}^{\max }$ are the minimum and maximum reactive power generation of the $j$ th capacitor.

4.2. Determination of Dependent Variables. After having the location and size of capacitors, backward/forward sweep (BFS) technique [64] is run to solve the power flow to obtain branch 
current and node voltage, called $I_{i, s}$ and $U_{i, s}$ in which $I_{i, s}$ is current flowing in the $i$ th branch corresponding to the sth solution and $U_{i, s}$ is voltage at the $i$ th node corresponding to the sth solution. Then, active power loss in each branch is calculated.

4.3. New Solution Generation and Correction. When applying the proposed method, new solutions are produced to update control variables for three times corresponding to the applications of diffusion technique, the first update technique, and the second update technique. After producing new solutions and before running the BFS technique, the new solutions must be checked and corrected based on the formula below:

$$
X_{s}= \begin{cases}X_{s}, & \text { if } X^{\min } \leq X_{s} \leq X^{\max }, \\ X^{\min }, & \text { if } X_{s}<X^{\min } ; s=1, \ldots, N_{\mathrm{Po}}, \\ X^{\max }, & \text { else. }\end{cases}
$$

In the equation above, control variables that are smaller than minimum values are set to the minimum values while other ones with higher value than the maximum values are set to the maximum values. For remaining control variables within the minimum and maximum values, the correction will not be executed. Because there is no violation of control variables, control variables are always accepted. However, valid control variables are not called optimal solutions. Optimal solutions must have valid control variables, valid dependent variables, and suitable objective function value.

4.4. Fitness Function. Fitness function must be calculated to reflect the quality of solutions. In the problem, fitness function is the sum of objective function and penalty terms, in which objective function (obj) can be either TPL in equation (1) or TC in equation (2), whereas the penalty terms are always the penalty of the violation of the branch current and penalty of the violation of node voltage [62].

$$
\begin{aligned}
\mathrm{Fit}_{s}= & \mathrm{obj}+\mathrm{PF}_{1}\left(\sum_{i=1}^{\mathrm{Nbr}}\left(\Delta I_{i, s}\right)^{2}\right) \\
& +\mathrm{PF}_{2}\left(\sum_{i=1}^{\mathrm{Nb}}\left(\Delta U_{i, s}\right)^{2}\right) ; \quad s=1, \ldots, N_{\mathrm{Po}} .
\end{aligned}
$$

In the fitness function, $\Delta I_{i, s}$ and $\Delta U_{i, s}$ are the violation of branch current in the $i$ th branch and the violation of voltage at the $i$ th node corresponding to the sth solution. The two factors are calculated as follows:

$$
\begin{aligned}
\Delta I_{i, s} & =\left\{\begin{array}{ll}
0, & \text { if } I_{i, s} \leq I_{i}^{\max }, \\
I_{i, s}-I_{i}^{\max }, & \text { else, }
\end{array} \quad\right. \\
\Delta U_{i, s} & = \begin{cases}0, & \text { if } U_{\min } \leq U_{i, s} \leq U_{\max }, \ldots, \mathrm{Nb}, \\
U_{\min }-U_{i, s}, & \text { if } U_{i, s}<U_{\min } ; i=1, \ldots, \mathrm{Nb}, \\
U_{i, s}-U_{\max }, & \text { else. }\end{cases}
\end{aligned}
$$

Equations (19) and (20) indicate that penalty terms can be equal to 0 if current is not higher than the maximum value of conductor and voltage of nodes is within the operating range of loads. In this case, the fitness function and the objective function are the same and the considered solution can be called valid solution. Nevertheless, the selection of an optimal solution needs a predetermined solution set and the best solution is then decided by using the lowest fitness value of all solutions. In the paper, fifty valid solutions are found for each study case and then the best solution is taken from the fifty available solutions.

4.5. The Procedure of Implementing MSFS for the Problem. The whole procedure of applying the proposed MSFS for finding the best site and size of capacitors can be plotted in Figure 1 and described in detail as follows:

Step 1: set values to $N_{\mathrm{Po}}, N_{\mathrm{Iter}}, N_{\mathrm{Df}}$, and DF.

Step 2: produce initial solutions satisfying constraint in equation (15).

Step 3: solve power flow by running the BFS technique to obtain $I_{i, s}$ and $U_{i, s}$.

Step 4: find fitness function as shown in equations (18)-(20).

Step 5: determine Gbest and set $C_{\text {Iter }}=1$.

Step 6: perform the diffusion technique using equation (12) to produce new solutions.

Step 7: correct violation of new solutions by using equation (17).

Step 8: solve power flow by running the BFS technique to obtain $I_{i, s}$ and $U_{i, s}$.

Step 9: find fitness function as shown in equations (18)-(20).

Step 10: compare new and old solutions to keep higher quality ones.

Step 11: sort the population in ascending order of fitness value.

Step 12: calculate average fitness function of the population Fit mean $_{\text {. }}$

Step 13: update new solutions for the first half of population using equation (13).

Step 14: correct violation of new solutions by using equation (17).

Step 15: solve power flow by running the BFS technique to obtain $I_{i, s}$ and $U_{i, s}$.

Step 16: calculate fitness function as shown in equations (18)-(20).

Step 17: at each position in the population, compare solutions to keep better one.

Step 18: sort all solutions in ascending order of fitness function.

Step 19: calculate average fitness function of the population Fit $_{\text {mean }}$.

Step 20: update new solutions for the first half of population using equation (13). 


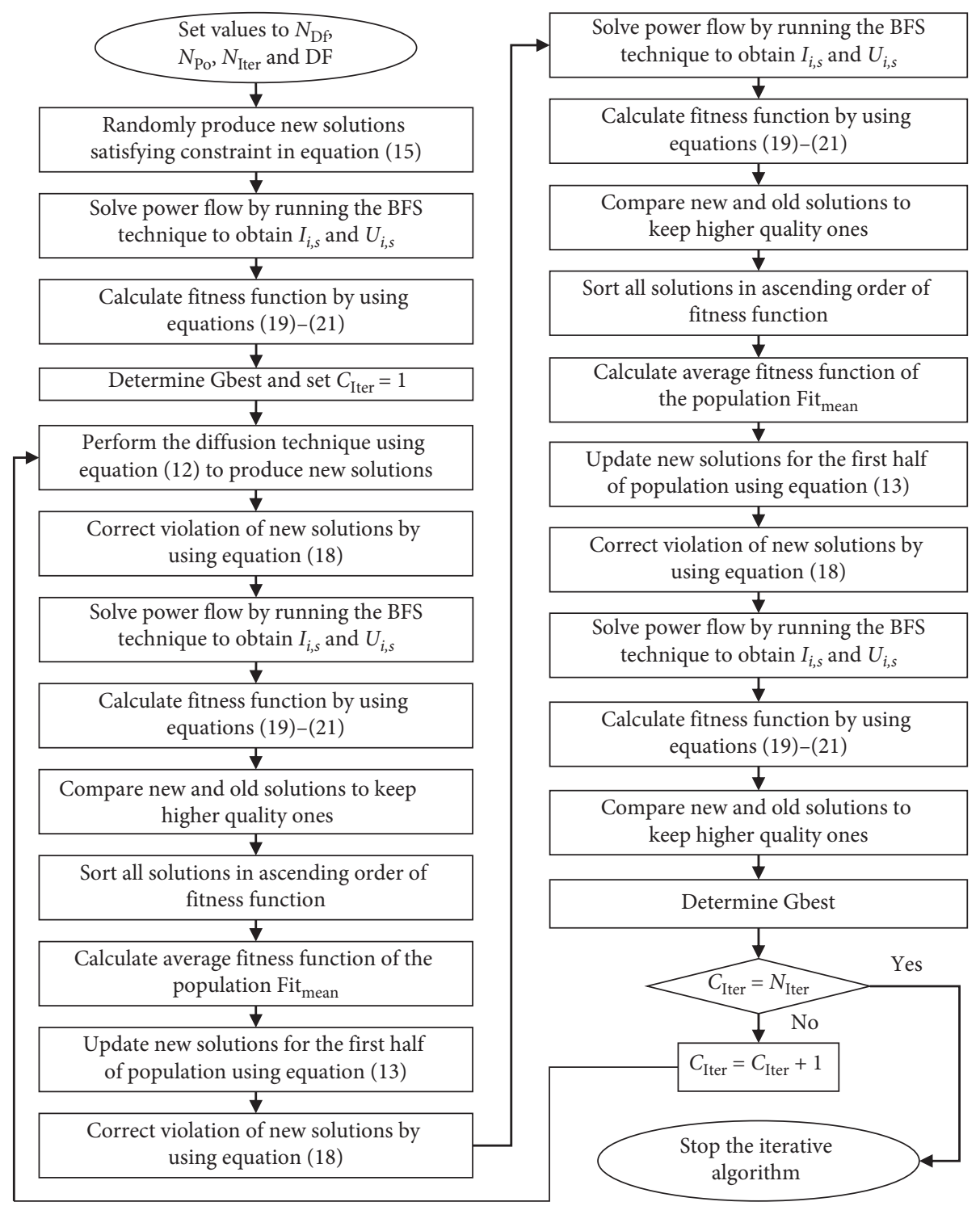

FIGURE 1: The MFS method implementation procedure for capacitor placement in distribution systems.

Step 21: correct violation of new solutions by using equation (17).

Step 22: solve power flow by running the BFS technique to obtain $I_{i, s}$ and $U_{i, s}$.

Step 23: find fitness function as shown in equations (18)-(20).

Step 24: at each position in the population, compare solutions to keep better one.

Step 25: determine Gbest.

Step 26: if $C_{\text {Iter }}=N_{\text {Iter }}$, stop the iterative algorithm. Otherwise, set $C_{\text {Iter }}=C_{\text {Iter }}+1$ and go back to step 6 .

\section{Numerical Results}

In this section, the performance of the proposed MSFS is investigated by implementing the capacitor bank placement in three distribution systems with the increase of bus number, from 33, 69 to 85. In addition, three other methods including the conventional SFS, SFS with the new diffusion technique (called MSFS1), and SFS with the two new update techniques (called MSFS2) are also executed for clearly showing the robustness of each new proposed technique. All the implemented methods are coded in Matlab program language and executed on a PC with a $2.4 \mathrm{GHz}$ processor and a $4.0 \mathrm{~GB}$ RAM. For each study case, each method is implemented for 50 trial runs.

For demonstration of the high performance of the proposed MSFS method, we focus on the comparison criteria as follows:

(1) The best solution of 50 trial runs: the four implemented methods are based on randomization, so the best solution should be collected for indicating the strong search ability of compared method. As compared to other previous methods, the best solution comparison can be performed by using the total power loss and total cost. 
(2) The fluctuations of 50 trial runs: the fluctuations can be reflected by observing the mean loss of 50 runs, the standard deviation of 50 runs, and the detail of 50 runs. Methods with lower mean and lower standard deviation can reach more stable search. The low fluctuations of 50 runs can confirm the lower mean and standard deviation obviously. As compared to other previous methods, mean power loss, maximum power loss, and standard deviation can be used for comparison; however, approximately all previous studies have ignored these values for comparisons. For the objective of total cost, the comparisons cannot be done because all previous methods neglected mean and maximum cost.

(3) The convergence speed: the four implemented methods are run by setting the same control parameters (i.e., the same diffusion number, the same population, and the same number of iterations). So, the method that can reach the best solution with better quality is the faster one. In addition, calculation time (CT) is also employed to evaluate the convergence speed. So, the criterion is also applied for comparing with other previous methods. Methods with lower control parameters and/or shorter CT can be faster.

\subsection{Result Comparison for the 33-Bus Network with Power Loss} Objective. In this section, the first distribution system with 33 buses is employed for placing capacitors with the aim to reduce total power loss. The system is plotted in Figure 2 [38].

The load of the system is comprised of $3715 \mathrm{~kW}$ and $2300 \mathrm{kVAr}$. The whole data of the system are taken from the study [38] and also reported in Table 1 . In order to obtain simulation results, four methods consisting of SFS, MSFS1, MSFS2, and the proposed MSFS are run by setting $N_{\mathrm{Df}}, N_{\mathrm{Po}}$, and $N_{\text {Iter }}$ to 2,10 , and 15 , respectively. The two study cases for the system corresponding to the different number of capacitor banks are shown in the following sections.

5.1.1. Case 1: Two Capacitor Banks $(\mathrm{Nc}=2)$. The summary of the 50 runs including minimum and standard deviation and the mean and maximum loss is, respectively, reported in Figures 3 and 4 while power loss from 50 runs found by the four executed methods is shown in Figure 5. Figure 3 shows that three modified methods can reduce the same total power loss to $141.8433 \mathrm{~kW}$ while SFS cannot reach the value although the loss is insignificantly higher. However, the standard deviation of the proposed method is the lowest with 0.78 and that of SFS is the highest with 2.19. As shown in Figure 4, the mean loss of the proposed method is the lowest and equal to $142.0859 \mathrm{~kW}$, whereas that of SFS is $143.86 \mathrm{~kW}$ and the highest. The proposed method can reach the second-best maximum loss with $145.9195 \mathrm{~kW}$ while SFS is still the worst method with the highest maximum loss with $151.23 \mathrm{~kW}$. Namely, the proposed method can reach less mean loss and less maximum loss than SFS by 1.77 and $5.31 \mathrm{~kW}$, respectively. Clearly, the performance of SFS

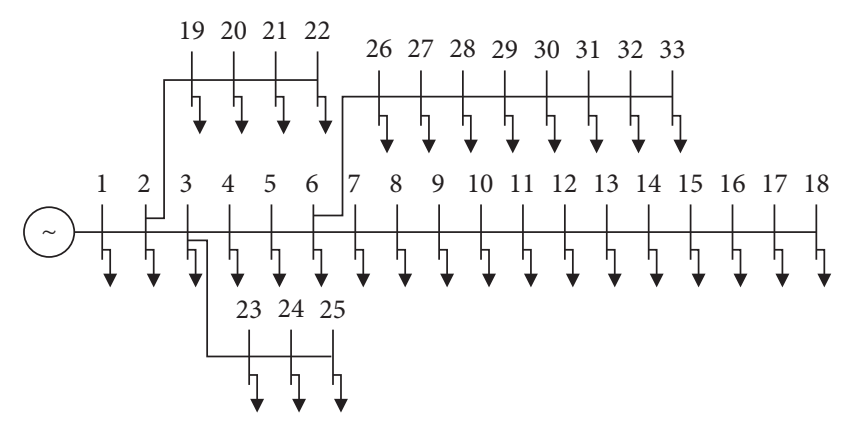

Figure 2: The 33-bus distribution system.

is the lowest among four implemented methods. Figure 5 can indicate the lowest stability of SFS since points in green have the highest fluctuations. On the contrary, the proposed method with points in blue has the lowest fluctuations and there are many points with the same loss as the best point.

In summary, the comparison of results can indicate the advantages as follows:

(1) The newly proposed diffusion technique and the two newly proposed update techniques are really effective. In fact, MSFS1 and MSFS2 can find less minimum loss, less mean loss, and less maximum loss than SFS. In addition, the standard deviation and the fluctuations of MSFS1 and MSFS2 are lower than those of SFS.

(2) The proposed method is much effective than its conventional method, SFS. In fact, the three comparison criteria are applied to indicate the statement exactly. The proposed method reaches less minimum loss, so it reaches superiority as shown in the first comparison criterion. The mean, maximum, and standard deviation of the proposed method are less than those of SFS. In addition, the proposed method can find many solutions with the same quality as the best solution. So, the second comparison criterion is confirmed. The proposed method and SFS use the same control parameters, but the proposed method can reach much better results. Hence, the proposed method is faster than SFS as said in the third comparison criterion.

In addition, the proposed method is also compared to previous methods as shown in Table 2. The results indicate that the proposed method can reach the smallest power loss with $141.8433 \mathrm{~kW}$ while two other methods reach higher loss with 141.94 and $141.9 \mathrm{~kW}$. As compared to the base network without capacitors, the loss reduction from HM [45], NCB-HM [42], and the proposed method is, respectively, $32.72 \%, 32.74 \%$, and $32.77 \%$. The calculation time of the proposed method is only 0.382 seconds, whereas the time of the two compared methods was not reported for comparison. It is noted that the time of 0.382 seconds is very small while the two methods have applied analytical methods. Furthermore, HM has used the power loss sensitivity factor to find the most appropriate location and then it has used PSO to search the size of each selected capacitor bank. So, the two methods are hardly ever faster than the 
Table 1: Data of the first system with 33 buses.

\begin{tabular}{|c|c|c|c|c|c|c|c|}
\hline \multirow[t]{2}{*}{ Line } & \multirow[t]{2}{*}{ Sending node } & \multirow[t]{2}{*}{ Receiving node } & \multirow[t]{2}{*}{ Resistance $(\Omega)$} & \multirow[t]{2}{*}{ Reactance $(\Omega)$} & \multicolumn{2}{|c|}{$\begin{array}{l}\text { Load at receiving } \\
\text { node }\end{array}$} & \multirow[t]{2}{*}{ Maximum line capacity (kVA) } \\
\hline & & & & & $P(\mathrm{~kW})$ & Q (kVAr) & \\
\hline 1 & 1 & 2 & 0.0922 & 0.047 & 100 & 60 & 400 \\
\hline 2 & 2 & 3 & 0.493 & 0.251 & 90 & 40 & 400 \\
\hline 3 & 3 & 4 & 0.3661 & 0.1864 & 120 & 80 & 400 \\
\hline 4 & 4 & 5 & 0.3811 & 0.1941 & 60 & 30 & 400 \\
\hline 5 & 5 & 6 & 0.819 & 0.707 & 60 & 20 & 400 \\
\hline 6 & 6 & 7 & 0.1872 & 0.6188 & 200 & 100 & 300 \\
\hline 7 & 7 & 8 & 1.7117 & 1.2357 & 200 & 100 & 300 \\
\hline 8 & 8 & 9 & 1.0299 & 0.74 & 60 & 20 & 200 \\
\hline 9 & 9 & 10 & 1.044 & 0.74 & 60 & 20 & 200 \\
\hline 10 & 10 & 11 & 0.1967 & 0.0651 & 45 & 30 & 200 \\
\hline 11 & 11 & 12 & 0.3744 & 0.1237 & 60 & 35 & 200 \\
\hline 12 & 12 & 13 & 1.468 & 1.1549 & 60 & 35 & 200 \\
\hline 13 & 13 & 14 & 0.5416 & 0.7129 & 120 & 80 & 200 \\
\hline 14 & 14 & 15 & 0.5909 & 0.526 & 60 & 10 & 200 \\
\hline 15 & 15 & 16 & 0.7462 & 0.5449 & 60 & 20 & 200 \\
\hline 16 & 16 & 17 & 1.2889 & 1.721 & 60 & 20 & 200 \\
\hline 17 & 17 & 18 & 0.732 & 0.5739 & 90 & 40 & 200 \\
\hline 18 & 2 & 19 & 0.164 & 0.1565 & 90 & 40 & 200 \\
\hline 19 & 19 & 20 & 1.5042 & 1.3555 & 90 & 40 & 200 \\
\hline 20 & 20 & 21 & 0.4095 & 0.4784 & 90 & 40 & 200 \\
\hline 21 & 21 & 22 & 0.7089 & 0.9373 & 90 & 40 & 200 \\
\hline 22 & 3 & 23 & 0.4512 & 0.3084 & 90 & 50 & 200 \\
\hline 23 & 23 & 24 & 0.898 & 0.7091 & 420 & 200 & 200 \\
\hline 24 & 24 & 25 & 0.8959 & 0.701 & 420 & 200 & 200 \\
\hline 25 & 6 & 26 & 0.2031 & 0.1034 & 60 & 25 & 300 \\
\hline 26 & 26 & 27 & 0.2842 & 0.1447 & 60 & 25 & 300 \\
\hline 27 & 27 & 28 & 1.0589 & 0.9338 & 60 & 20 & 300 \\
\hline 28 & 28 & 29 & 0.8043 & 0.7006 & 120 & 70 & 200 \\
\hline 29 & 29 & 30 & 0.5074 & 0.2585 & 200 & 600 & 200 \\
\hline 30 & 30 & 31 & 0.9745 & 0.9629 & 150 & 70 & 200 \\
\hline 31 & 31 & 32 & 0.3105 & 0.3619 & 210 & 100 & 200 \\
\hline 32 & 32 & 33 & 0.3411 & 0.5302 & 60 & 40 & 200 \\
\hline
\end{tabular}

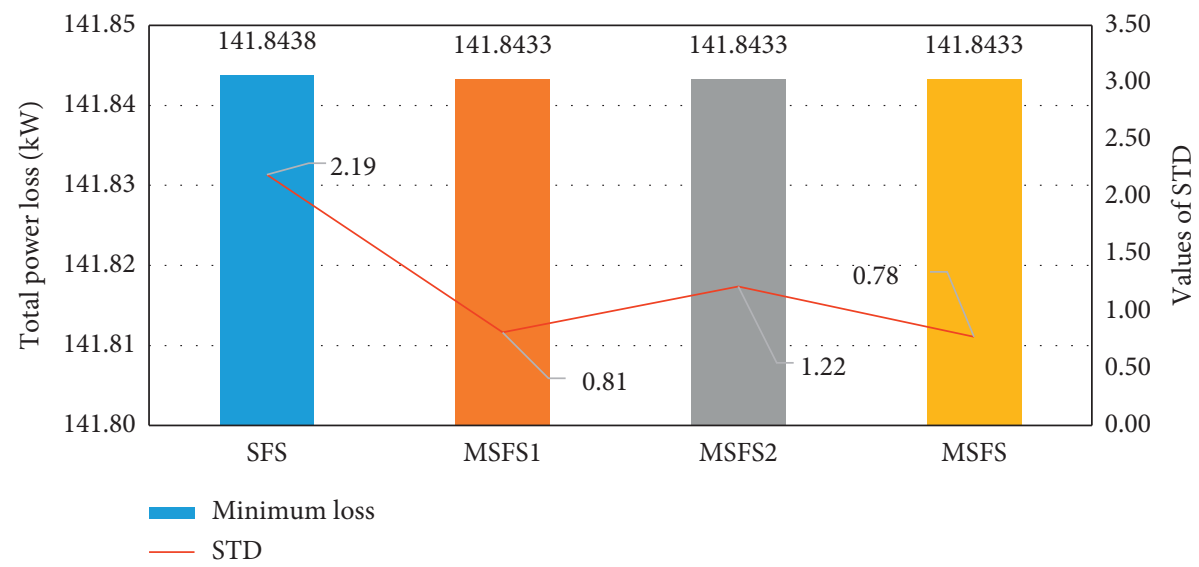

FIGURE 3: Minimum power loss and standard deviation of 50 trial runs obtained by SFS methods for case 1 of the 33-bus system.

proposed method. So, the proposed method is superior to the two methods for the study case.

5.1.2. Case 2: Three Capacitor Banks $(\mathrm{Nc}=3)$. The results from 50 trial runs for the study case with 3 capacitor banks are shown in Figures 6-8. The observation from Figures 6 and 7 shows the same characteristic that the height of bars tends to be decreased from SFS to MSFS. This means the improvement is gradually increased from MSFS1, MSFS2 to MSFS, whereas SFS is the worst method with the highest bars. The exact comparisons indicate that the proposed can find less minimum loss, less mean loss, and less maximum 


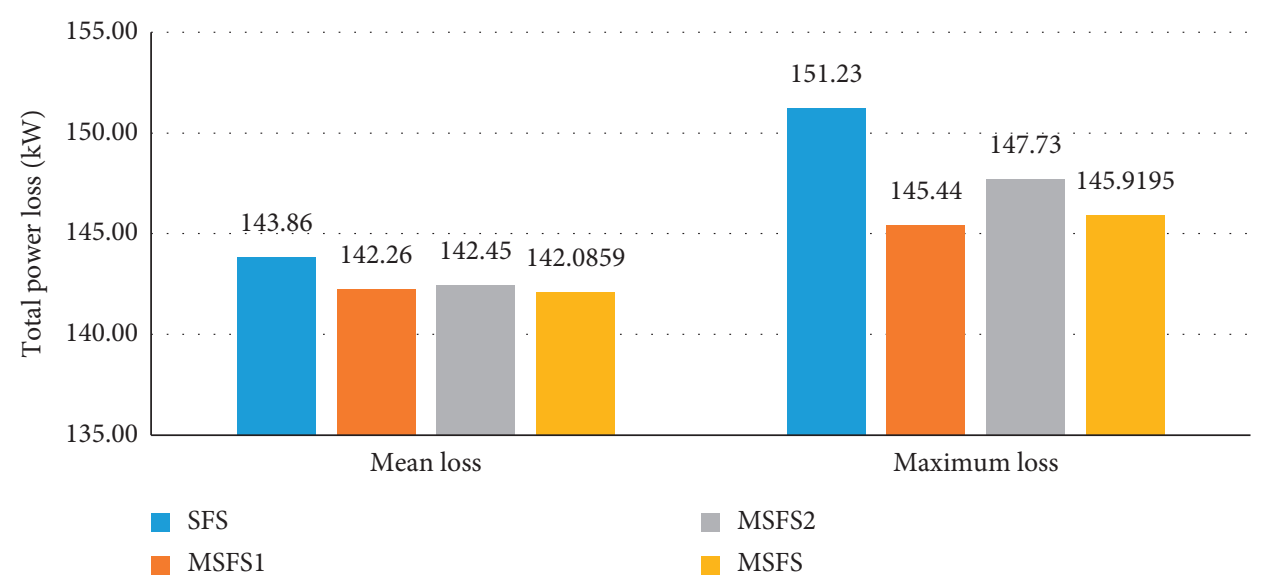

Figure 4: Mean and maximum power loss of 50 trial runs obtained by SFS methods for case 1 of the 33-bus system.

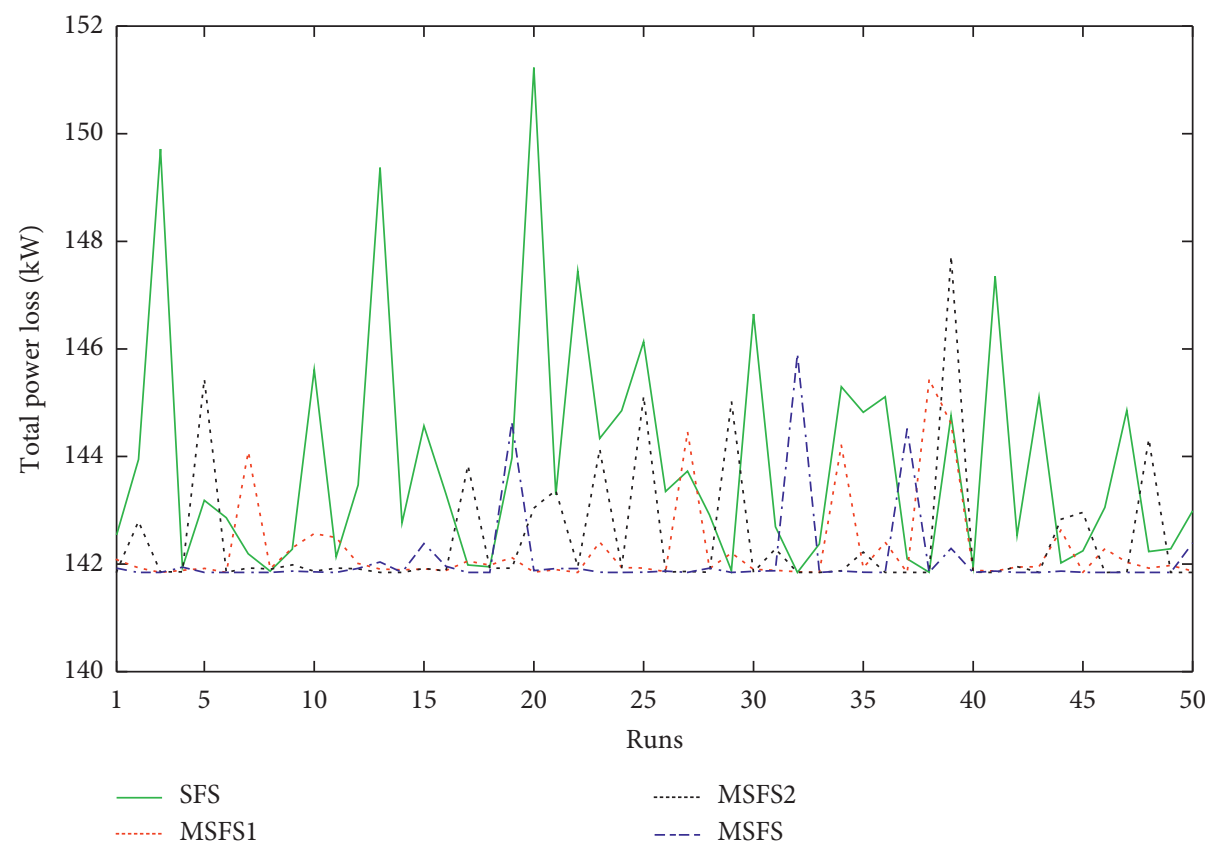

Figure 5: Total power loss of 50 trial runs obtained by SFS methods for case 1 of the 33-bus system.

TABle 2: Comparison with other methods for case 1 of the 33-bus system.

\begin{tabular}{|c|c|c|c|c|c|c|c|c|c|c|}
\hline Method & $N_{\text {Po }}$ & $N_{\text {Iter }}$ & Bus & Size & $\begin{array}{c}\text { Total capacity } \\
\text { (kVAr) }\end{array}$ & Min. loss $(\mathrm{kW})$ & Mean loss $(\mathrm{kW})$ & Max. loss $(\mathrm{kW})$ & STD & $\mathrm{CT}(\mathrm{s})$ \\
\hline $\begin{array}{l}\text { Base network } \\
(\mathrm{Nc}=0)\end{array}$ & - & - & - & - & - & 211 & - & - & - & - \\
\hline NCB-HM [42] & - & - & $\begin{array}{l}13 \\
30\end{array}$ & $\begin{array}{c}405 \\
1052\end{array}$ & 1457 & 141.9 & - & - & - & - \\
\hline HM [45] & - & - & $\begin{array}{l}12 \\
30\end{array}$ & $\begin{array}{c}430 \\
1040\end{array}$ & 1470 & 141.94 & - & - & - & - \\
\hline MSFS & 10 & 15 & $\begin{array}{l}12 \\
30\end{array}$ & $\begin{array}{l}465.20 \\
1063.3\end{array}$ & 1528.5 & 141.8433 & 142.0859 & 145.9195 & 0.7757 & 0.382 \\
\hline
\end{tabular}

loss than SFS by $0.26 \mathrm{~kW}, 2.35 \mathrm{~kW}$, and $4.76 \mathrm{~kW}$, respectively. In addition, the standard deviation of the proposed method is also smaller than that of SFS by 0.86 . Clearly, the new diffusion technique and the two new update techniques have a significant impact on the improvement of the proposed method in finding better solutions and in stabilizing the search process. Figure 8 can show clear advantage of the proposed method since approximately all points of the proposed method have smaller fluctuations and have lower loss than those of the SFS method. Furthermore, the 
proposed method can find eight solutions with the same quality as the best solution, but SFS cannot find even one run with the same loss as the best solution.

There is a main difference of the case with 3 capacitor banks and above case with 2 capacitor banks in finding the best solution. Only MSFS can find the best solution for this case while other ones are searching solutions. But the four methods could find the same solutions for the above case. The fact that this case is more complicated is due to the higher number of control variables (i.e., 3 capacitor banks are corresponding to six control variables, three locations, and three sizes). So, SFS as well as MSFS1 and MSFS2 needs more iterations for finding the same solution as the proposed MSFS method.

For giving more evidences of the high performance of the proposed method, Table 3 is established for comparisons with previous methods. Looking through the minimum loss in the table, we can see that the lowest minimum loss is from MSFS with $138.31 \mathrm{~kW}$ while the second-lowest minimum loss is from HM [45] and CPM [44] with $138.37 \mathrm{~kW}$ and the highest minimum loss is from BFOA [30] with $144.04 \mathrm{~kW}$. The exact calculation indicates the proposed method can reduce the loss from $0.06 \mathrm{~kW}$ to $5.72 \mathrm{~kW}$ as compared to other ones. As compared to the base network without capacitor banks, the proposed method can reach the total loss reduction of $34.45 \%$ while other ones can reach the reduction from $31.73 \%$ to $34.42 \%$. Clearly, the improvement of the proposed method is higher than that of other ones from $0.03 \%$ to $2.72 \%$. The comparison of convergence speed is not successful excluding the comparison with BFOA [30] and FPA [33]. Population size and the number of iterations from the proposed method are less than those from the two methods. They are 10 for the proposed method, but they are 50 for BFOA [30] and 20 and 200 for FPA [33]. The simulation time of FPA was 7.75 seconds, but it is only 0.36 seconds for the proposed MSFS method. In summary, the proposed method can reach better solution than other methods and its speed can be as fast as them or much faster than them. Consequently, the proposed method is an effective one for the study case with $3 \mathrm{ca}$ pacitor banks in the 33-node distribution system.

The voltage profile of the 33-node distribution system for three cases, the first case without capacitor placement (called Base), the second case with 2 capacitors, and the third case with 3 capacitors is plotted in Figure 9. The three curves show the significant improvement of voltage for the cases with capacitor bank placement. The lowest voltage is much increased from less than $0.91 \mathrm{pu}$ of the base network to higher than $0.93 \mathrm{pu}$ of the two cases with capacitors. Furthermore, voltage of approximately all nodes of the cases with capacitors is higher than that of the base network. Clearly, the capacitor placement is necessary for the voltage profile improvement purpose. However, the improvement of the case with 3 capacitors over the case with 2 capacitors is not much since the same nodes from the two cases have approximately the same voltage excluding nodes 23,24 , and 25 where the case with 3 capacitors has better voltage.
5.2. Result Comparison for the 69-Bus Network with Power Loss Objective. In this section, the four executed methods are tested on a larger scale system with 69 nodes as shown in Figure 10 [38]. The total active power and reactive power of all loads are, respectively $3801 \mathrm{~kW}$ and $2695 \mathrm{kVAr}$. The data of the system are taken from the study [38] and also reported in Table 4. In order to solve system with two cases, two capacitor banks and three capacitor banks, the four executed methods are run by setting $N_{\mathrm{Df}}, N_{\mathrm{Po}}$, and $N_{\text {Iter }}$ to 2,10 , and 20 , respectively. The simulation result comparisons are presented in the following sections.

5.2.1. Case 1: Two Capacitor Banks $(N c=2)$. The minimum loss and standard deviation and the mean loss and maximum loss from the four methods are given in Figures 11 and 12. The minimum loss of the study case is similar to the case with 2 capacitor banks of the 33-node distribution system since MSFS1, MSFS2, and the proposed MSFS have approximately the same loss about $146.44 \mathrm{~kW}$ while that of SFS is much higher and equal to $146.6221 \mathrm{~kW}$. For other results, the proposed method continues to show outstanding performance because it gets the lowest values of STD, mean loss, and maximum loss. SFS is still the worst method with all highest values. The exact comparison between SFS and the proposed method shows that the proposed method can reach smaller minimum, mean, and maximum loss than SFS by $0.18,3.45$, and $7.33 \mathrm{~kW}$, respectively. SFS is less stable in searching solution since its standard deviation is higher by 1.67. In addition, Figure 13 is also a clear evidence for the improvement of the proposed method over SFS. The fluctuations of the proposed method are much smaller than those of SFS, and MSFS can find more than thirty best solutions, but SFS cannot find the best solution even one time. Consequently, the proposed method outperforms SFS in effectively finding solution and converging faster to the best solution.

The proposed method is compared to other ones in Table 5. Because mean loss, maximum loss, STD, and CT for all methods were not reported, the comparison for further investigation failed. However, the minimum loss can be seen from the best performance of the proposed method since it reaches the lowest loss with $146.44 \mathrm{~kW}$ while that of the base network, the second-best method, and the worst method is $225,146.5$, and $152.05 \mathrm{~kW}$. Obviously, the proposed method can reduce loss by $78.56,0.06$, and $5.6141 \mathrm{~kW}$, respectively. The proposed method can reduce power loss up to $34.9 \%$ compared to the base network without capacitor placement, and its improvement level over other ones is from 0.04 to $3.69 \%$. These values can show the high performance of the proposed method. NCB-HM [42] and HM [45] have reached results as good as the proposed method, but these methods have been based on the features of networks and convergence speed cannot be measured. PSO and GA are much slower than the proposed method. The simulation time of the two methods was not reported, but the population size and the number of iterations are very high, 30 and 50, while those are only 10 and 20 for the proposed method. So, the proposed method is very useful for the study case. 


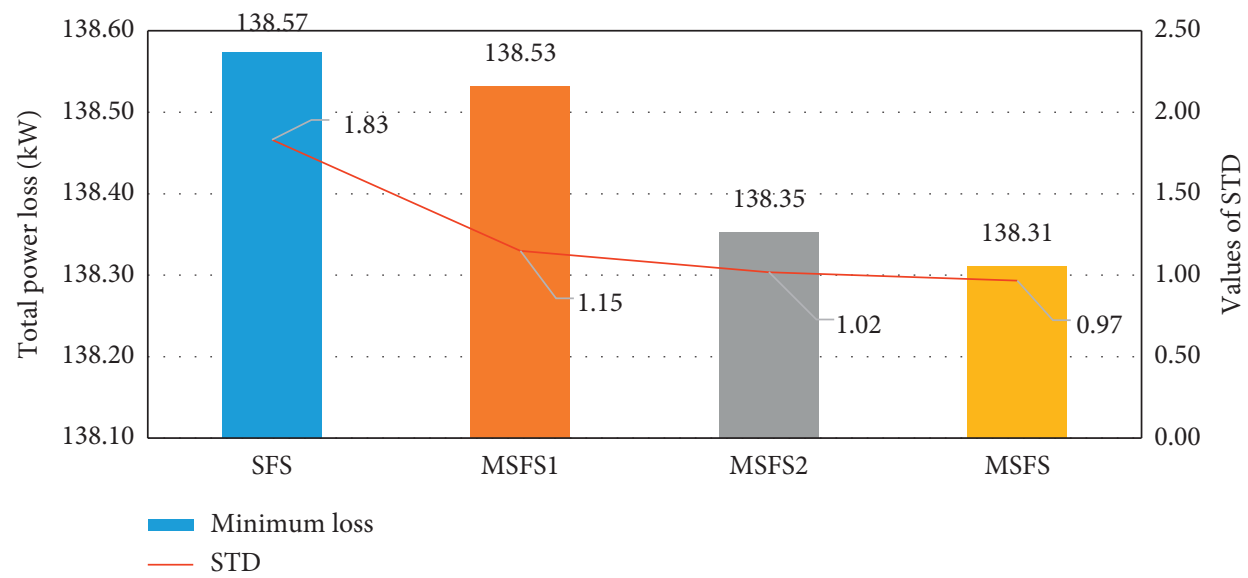

FIgURE 6: Minimum power loss and standard deviation of 50 trial runs obtained by SFS methods for case 2 of the 33-bus system.

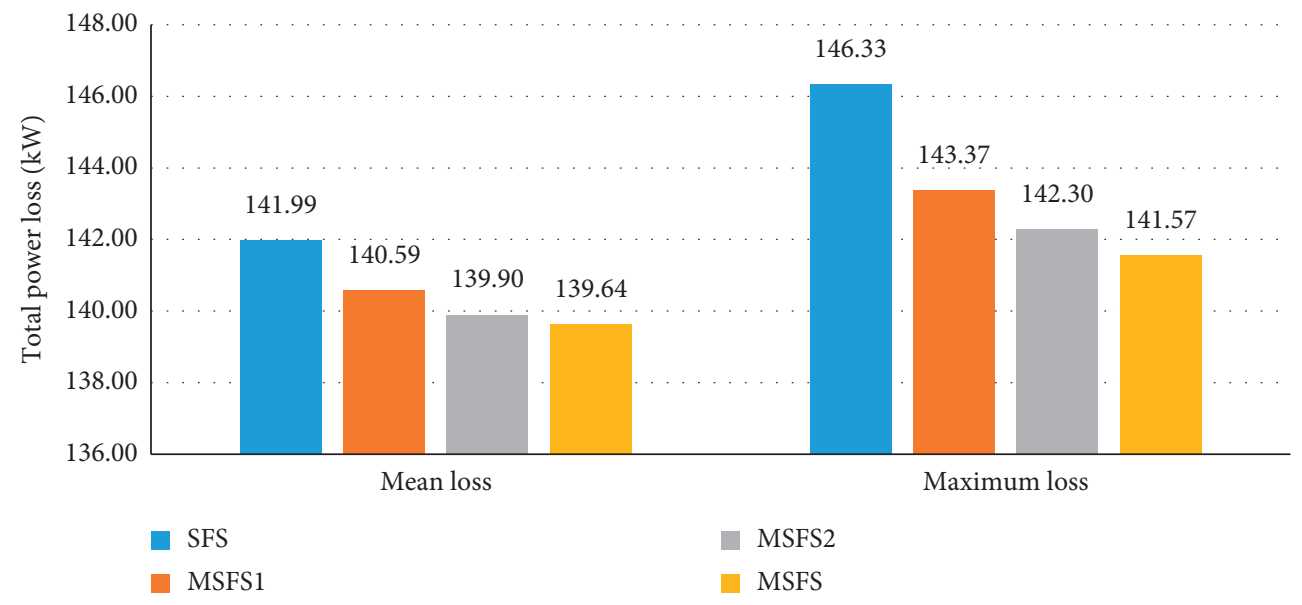

Figure 7: Mean and maximum power loss of 50 trial runs obtained by SFS methods for case 2 of the 33-bus system.

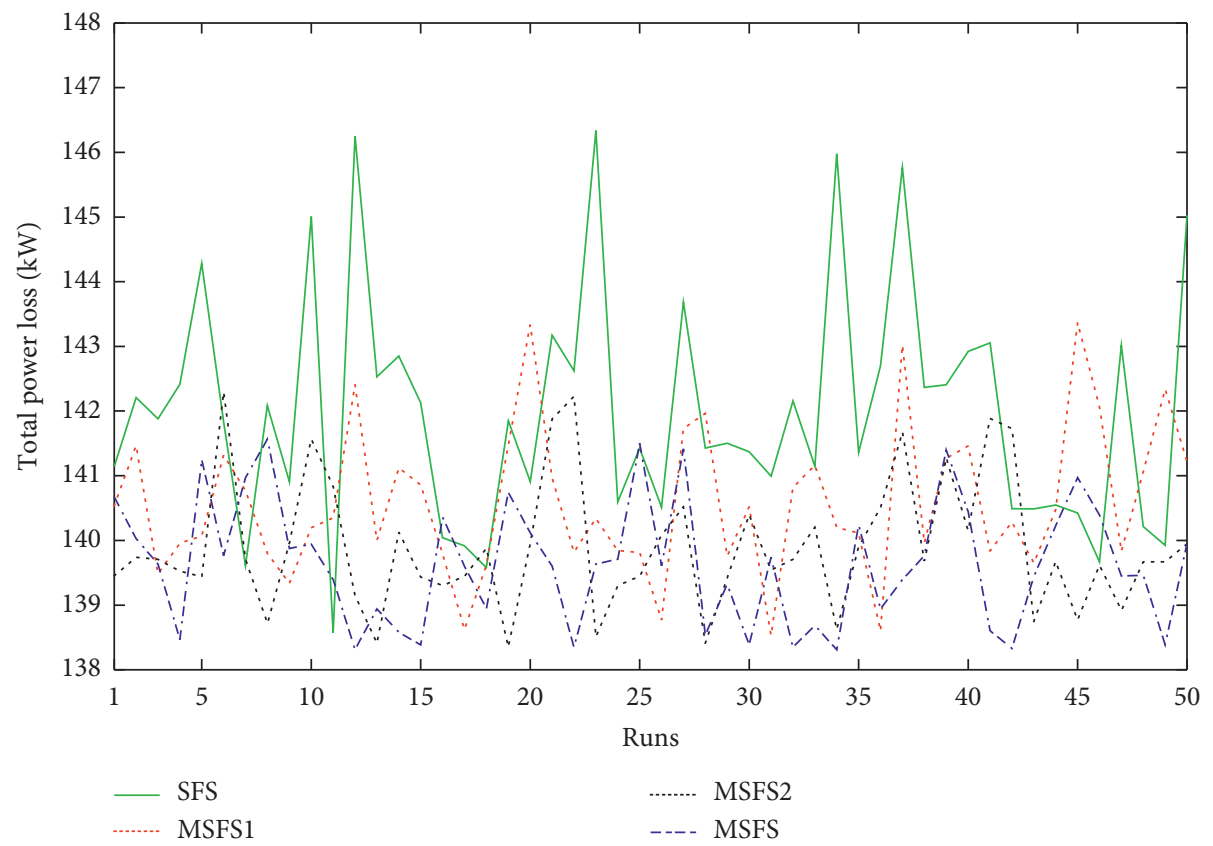

FIGURE 8: Total power loss of 50 trial runs obtained by SFS methods for case 2 of the 33-bus system. 
TABLE 3: Comparison with other methods for case 2 of the 33-bus system.

\begin{tabular}{|c|c|c|c|c|c|c|c|c|c|c|}
\hline Method & $N_{\text {Po }}$ & $N_{\text {Iter }}$ & Bus & Size & $\begin{array}{c}\text { Total capacity } \\
\text { (kVAr) }\end{array}$ & Min. loss (kW) & Mean loss $(\mathrm{kW})$ & Max. loss $(\mathrm{kW})$ & STD & $\mathrm{CT}(\mathrm{s})$ \\
\hline $\begin{array}{l}\text { Base network } \\
(\mathrm{Nc}=0)\end{array}$ & - & - & - & - & - & 211 & - & - & - & - \\
\hline BFOA [30] & 50 & 50 & $\begin{array}{l}18 \\
30 \\
33 \\
13\end{array}$ & $\begin{array}{c}349.6 \\
820.6 \\
277.3 \\
450\end{array}$ & 1447.5 & 144.04 & - & - & - & - \\
\hline FPA [33] & 20 & 200 & $\begin{array}{l}24 \\
30 \\
13\end{array}$ & $\begin{array}{l}450 \\
900 \\
383\end{array}$ & 1800 & 139.075 & 13139.57 & 140.046 & 0.07793 & 7.75 \\
\hline NCB-HM [42] & NR & NR & $\begin{array}{l}25 \\
30 \\
12\end{array}$ & $\begin{array}{c}386 \\
1000 \\
500\end{array}$ & 1769 & 138.65 & NR & NR & NR & - \\
\hline CPM [43] & NR & $\mathrm{NR}$ & $\begin{array}{l}24 \\
30 \\
13\end{array}$ & $\begin{array}{c}500 \\
1000 \\
359\end{array}$ & 2000 & 138.61 & NR & NR & NR & - \\
\hline CPM [44] & NR & NR & $\begin{array}{l}24 \\
30 \\
13\end{array}$ & $\begin{array}{c}520 \\
1016 \\
360\end{array}$ & 1895 & 138.37 & NR & NR & NR & - \\
\hline HM [45] & NR & NR & $\begin{array}{l}24 \\
30 \\
12\end{array}$ & $\begin{array}{c}510 \\
1020 \\
436.2\end{array}$ & 1890 & 138.37 & - & - & - & - \\
\hline MSFS & 10 & 15 & $\begin{array}{l}24 \\
30\end{array}$ & $\begin{array}{c}538.4 \\
1015\end{array}$ & 1989.6 & 138.31 & 139.6427 & 141.5659 & 0.9661 & 0.37 \\
\hline
\end{tabular}

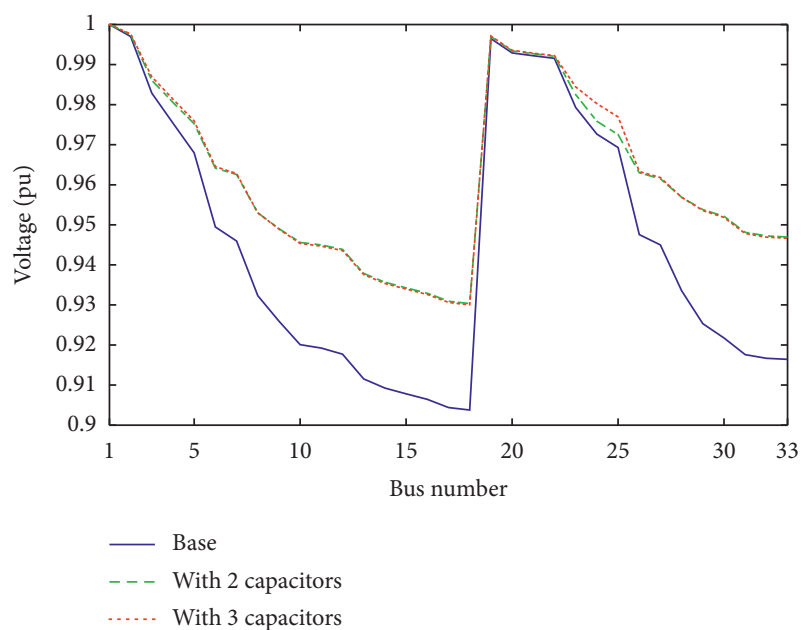

Figure 9: Voltage profile of the 33-bus network for different cases of capacitor placement.

5.2.2. Case 2: Three Capacitor Banks $(N c=3)$. For the case, the minimum loss, standard deviation, and the mean loss have the same shape as shown in Figures 14 and 15, and only maximum loss has a slight difference. In fact, the bars in the figures tend to be shorter gradually from SFS to MSFS1, MSFS2, and MSFS. The minimum loss is, respectively, $145.59,145.19,145.14$, and 145.13 for SFS, MSFS1, MSFS2, and MSFS. Similarly, the standard deviation is also decreased from 1.61 to $1.31,1.24$, and 1.12 for SFS, MSFS1, MSFS2, and MSFS. The mean loss is the highest for SFS with $147.88 \mathrm{~kW}$ and is the lowest for MSFS with $146.35 \mathrm{~kW}$. For the maximum loss, SFS is still the worst method with the loss of $152.33 \mathrm{~kW}$ while MSFS2 is the best method with the maximum loss of $151.24 \mathrm{~kW}$. The comparison with SFS indicates that the proposed method can reach the reduction of minimum loss, mean loss, and maximum loss by 0.46 , 1.53 , and $1.12 \mathrm{~kW}$. Figure 16 shows the strong search of the proposed method with many points having smaller loss than other ones, especially compared to SFS. The proposed method can find three best solutions with the lowest loss, whereas SFS cannot find the same quality solution over 50 runs. Consequently, the proposed method still reaches the best performance for the study case because it can find approximately all better results excluding the maximum loss.

Table 6 summarizes comparisons of the proposed method with other ones for the study case. The optimal location, size, and the minimum loss of all methods have been reported, but other results such as mean loss, maximum loss, standard deviation, and CT have been reported for only some methods. In addition, control parameters such as population and iterations have not been reported for all methods. The minimum loss of the compared methods is from $145.2 \mathrm{~kW}$ to $156.62 \mathrm{~kW}$ while the best loss of the proposed method is $145.13 \mathrm{~kW}$. Clearly, the proposed method can reduce the loss by from $0.07 \mathrm{~kW}$ to $11.49 \mathrm{~kW}$ as compared to other ones and by $79.87 \mathrm{~kW}$ as compared to the base network without capacitors. From the power loss reduction, the improvement level of the proposed MSFS method over the worst compared method and the best compared method can be calculated to be $7.34 \%$ and $0.05 \%$. Furthermore, the power loss improvement can be up to $35.5 \%$ as compared to the original network without the placement of capacitors. Only FPA [33] reported better mean loss, maximum loss, and standard deviation than the proposed method, but it used 20 for population size and 200 for iterations and took much longer calculation time, 18.36 


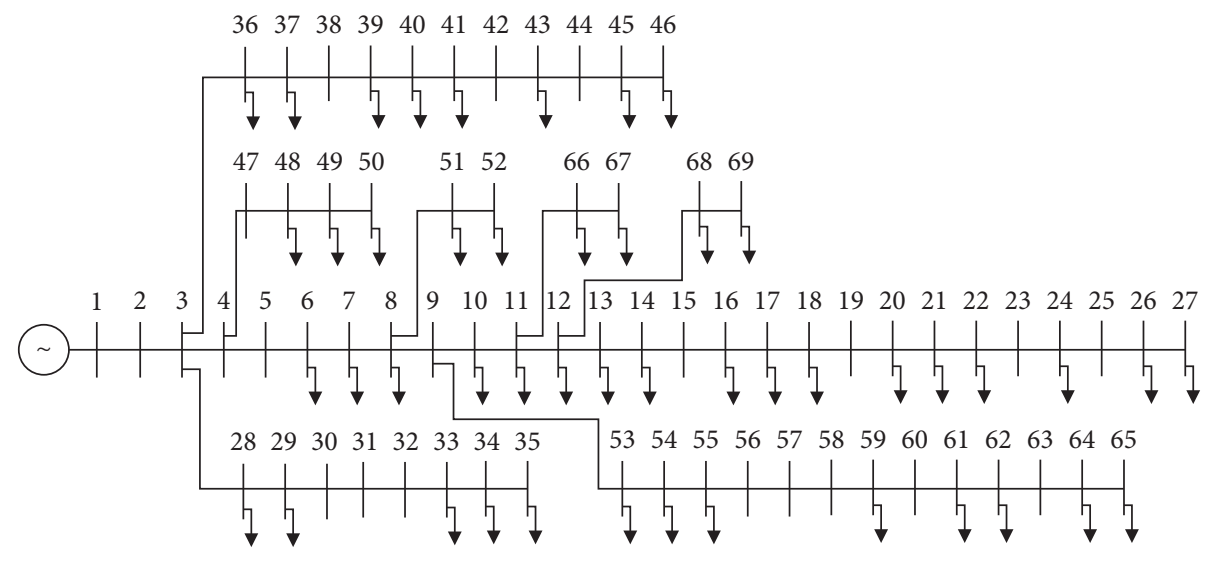

Figure 10: The 69-bus distribution system.

seconds. TLA [34] reported better maximum loss and standard deviation but worse minimum loss, worse mean loss, and longer simulation time together with higher population size and more iterations. The population size and iterations of the method are 50 and 100, but those of the proposed method are only 10 and 20 . The values resulted in very high simulation time of TLA with 15.76 seconds, which is much higher than 0.96 seconds of the proposed method. Clearly, the proposed method can be higher than five times faster than TLA through the comparison of control parameters and higher than sixteen times faster than TLA via the comparison of the simulation time. Other methods with the presentation of population and iterations are FGA [26] (60 and 100), GSA [28] (2500 and 750), and MSA [39] (50 and 100), but only MSA [39] reported simulation time of 11.42 seconds. Through the comparison of control parameter settings, the proposed method is, respectively, about 10 , 3125, and 8 times faster than FGA [26], GSA [28], and MSA [39]. These methods are much slower, but they find lower quality of optimal solutions. The result comparison analysis can confirm the following advantages of the proposed method.

(1) Reaching better minimum loss than all compared methods.

(2) Using smaller population size and smaller number of iterations than metaheuristics, whose control parameters were reported.

(3) Spending smaller calculation time than other methods.

As a result, it can be concluded that the proposed method is more suitable than other ones when applied for determining optimal location and size of capacitors in the 69-node distribution system.

Figure 17 shows the improvement of node voltage when installing capacitors in the 69-bus distribution system. The blue curve shows the voltage of all nodes before installing capacitors while the green and red curves are voltage of all nodes for the cases with two and three capacitors. The lowest voltage can be seen for the blue curve which is about less than 0.91 pu but that of the green curve and red curve is much higher, about higher than $0.93 \mathrm{pu}$. At other nodes, the improvement is not as high as at the lowest node voltage, but the improvement is significant. The deviation of voltage is high from bus 10 to bus 29 and from bus 57 to bus 66 . The deviation can confirm the high contribution of the capacitors in enhancing the voltage profile. The comparison between the case with 2 capacitors and 3 capacitors reveals that the voltage profile is not much improved when increasing the number of capacitors from 2 to 3 . Consequently, if the voltage profile is an objective, 3 capacitors cannot be the best option. This maybe dependent on other issues such as investment capital and operation cost.

5.3. Result Comparison for the 85-Bus Network with Power Loss Objective. In the section, the four methods are run on the largest distribution system with 85 buses and the number of capacitors is increased from 2, 3, 4 to 14 . The total active power and the total reactive power of all loads are $2570.28 \mathrm{~kW}$ and $2622.08 \mathrm{kVAr}$. The system is plotted in Figure 18 [35]. The entire data are taken from the study [35] and also given in Table 7. Due to the large system and the different number of control variables, $N_{\mathrm{Df}}, N_{\mathrm{Po}}$, and $N_{\text {Iter }}$ are set to different values for different cases. They are shown in detail in the following sections.

5.3.1. Case 1: Two Capacitor Banks $(N c=2)$. In order to solve the study case with 2 capacitors, the four executed methods are run by setting $N_{\mathrm{Df}}, N_{\text {Po }}$, and $N_{\text {Iter }}$ to 2,10 , and 25, respectively. All results are reported in Figures 19-21. The minimum loss in Figure 19 shows that all the methods have approximately the same best solution; however, the proposed method can reach the best solution with the lowest loss, which is slightly less than that of other ones. The standard deviation, 0.5669 for MSFS and 1.4205 for SFS, from the figure also shows the most stable search of MSFS and the worst stable search of SFS. Figure 20 continues to give the better result of MSFS since its mean loss and maximum loss are the smallest values, and SFS still reaches the highest value for the mean loss and the second-highest value for the maximum loss. The proposed method can reach less minimum loss, mean loss, and maximum loss than SFS 
TABle 4: Data of the second system with 69 buses.

\begin{tabular}{|c|c|c|c|c|c|c|c|}
\hline \multirow[t]{2}{*}{ Line } & \multirow[t]{2}{*}{ Sending node } & \multirow[t]{2}{*}{ Receiving node } & \multirow[t]{2}{*}{ Resistance $(\Omega)$} & \multirow[t]{2}{*}{ Reactance $(\Omega)$} & \multicolumn{2}{|c|}{$\begin{array}{c}\text { Load at receiving } \\
\text { node }\end{array}$} & \multirow[t]{2}{*}{ Maximum line capacity (kVA) } \\
\hline & & & & & $P(\mathrm{~kW})$ & $Q(\mathrm{kVAr})$ & \\
\hline 1 & 1 & 2 & 0.0005 & 0.0012 & 0 & 0 & 10761 \\
\hline 2 & 2 & 3 & 0.0005 & 0.0012 & 0 & 0 & 10761 \\
\hline 3 & 3 & 4 & 0.0015 & 0.0036 & 0 & 0 & 10761 \\
\hline 4 & 4 & 5 & 0.0251 & 0.0294 & 0 & 0 & 5823 \\
\hline 5 & 5 & 6 & 0.366 & 0.1864 & 2.6 & 2.2 & 1899 \\
\hline 6 & 6 & 7 & 0.3811 & 0.1941 & 40.4 & 30 & 1899 \\
\hline 7 & 7 & 8 & 0.0922 & 0.047 & 75 & 54 & 1899 \\
\hline 8 & 8 & 9 & 0.0493 & 0.0251 & 30 & 22 & 1899 \\
\hline 9 & 9 & 10 & 0.819 & 0.2707 & 28 & 19 & 1455 \\
\hline 10 & 10 & 11 & 0.1872 & 0.0691 & 145 & 104 & 1455 \\
\hline 11 & 11 & 12 & 0.7114 & 0.2351 & 145 & 104 & 1455 \\
\hline 12 & 12 & 13 & 1.03 & 0.34 & 8 & 5.5 & 1455 \\
\hline 13 & 13 & 14 & 1.044 & 0.345 & 8 & 5.5 & 1455 \\
\hline 14 & 14 & 15 & 1.058 & 0.3496 & 0 & 0 & 1455 \\
\hline 15 & 15 & 16 & 0.1966 & 0.065 & 45.5 & 30 & 1455 \\
\hline 16 & 16 & 17 & 0.3744 & 0.1238 & 60 & 35 & 1455 \\
\hline 17 & 17 & 18 & 0.0047 & 0.0016 & 60 & 35 & 2200 \\
\hline 18 & 18 & 19 & 0.3276 & 0.1083 & 0 & 0 & 1455 \\
\hline 19 & 19 & 20 & 0.2106 & 0.069 & 1 & 0.6 & 1455 \\
\hline 20 & 20 & 21 & 0.3416 & 0.1129 & 114 & 81 & 1455 \\
\hline 21 & 21 & 22 & 0.014 & 0.0046 & 5.3 & 3.5 & 1455 \\
\hline 22 & 22 & 23 & 0.1591 & 0.0526 & 0 & 0 & 1455 \\
\hline 23 & 23 & 24 & 0.3463 & 0.1145 & 28 & 20 & 1455 \\
\hline 24 & 24 & 25 & 0.7488 & 0.2745 & 0 & 0 & 1455 \\
\hline 25 & 25 & 26 & 0.3089 & 0.1021 & 14 & 10 & 1455 \\
\hline 26 & 26 & 27 & 0.1732 & 0.0572 & 14 & 10 & 1455 \\
\hline 27 & 3 & 28 & 0.0044 & 0.0108 & 26 & 18.6 & 10761 \\
\hline 28 & 28 & 29 & 0.064 & 0.1565 & 26 & 18.6 & 10761 \\
\hline 29 & 29 & 30 & 0.3978 & 0.1315 & 0 & 0 & 1455 \\
\hline 30 & 30 & 31 & 0.0702 & 0.0232 & 0 & 0 & 1455 \\
\hline 31 & 31 & 32 & 0.351 & 0.116 & 0 & 0 & 1455 \\
\hline 32 & 32 & 33 & 0.839 & 0.2816 & 14 & 10 & 2200 \\
\hline 33 & 33 & 34 & 1.708 & 0.5646 & 19.5 & 14 & 1455 \\
\hline 34 & 34 & 35 & 1.474 & 0.4673 & 6 & 4 & 1455 \\
\hline 35 & 3 & 36 & 0.0044 & 0.0108 & 26 & 18.55 & 10761 \\
\hline 36 & 36 & 37 & 0.064 & 0.1565 & 26 & 18.55 & 10761 \\
\hline 37 & 37 & 38 & 0.1053 & 0.123 & 0 & 0 & 5823 \\
\hline 38 & 38 & 39 & 0.0304 & 0.0355 & 24 & 17 & 5823 \\
\hline 39 & 39 & 40 & 0.0018 & 0.0021 & 24 & 17 & 5823 \\
\hline 40 & 40 & 41 & 0.7283 & 0.8509 & 1.2 & 1 & 5823 \\
\hline 41 & 41 & 42 & 0.31 & 0.3623 & 0 & 0 & 5823 \\
\hline 42 & 42 & 43 & 0.041 & 0.0478 & 6 & 4.3 & 5823 \\
\hline 43 & 43 & 44 & 0.0092 & 0.0116 & 0 & 0 & 5823 \\
\hline 44 & 44 & 45 & 0.1089 & 0.1373 & 39.22 & 26.3 & 5823 \\
\hline 45 & 45 & 46 & 0.0009 & 0.0012 & 39.22 & 26.3 & 6709 \\
\hline 46 & 4 & 47 & 0.0034 & 0.0084 & 0 & 0 & 10761 \\
\hline 47 & 47 & 48 & 0.0851 & 0.2083 & 79 & 56.4 & 10761 \\
\hline 48 & 48 & 49 & 0.2898 & 0.7091 & 384.7 & 274.5 & 10761 \\
\hline 49 & 49 & 50 & 0.0822 & 0.2011 & 384 & 274.5 & 10761 \\
\hline 50 & 8 & 51 & 0.0928 & 0.0473 & 40.5 & 28.3 & 1899 \\
\hline 51 & 51 & 52 & 0.3319 & 0.1114 & 3.6 & 2.7 & 2200 \\
\hline 52 & 9 & 53 & 0.174 & 0.0886 & 4.35 & 3.5 & 1899 \\
\hline 53 & 53 & 54 & 0.203 & 0.1034 & 26.4 & 19 & 1899 \\
\hline 54 & 54 & 55 & 0.2842 & 0.1447 & 24 & 17.2 & 1899 \\
\hline 55 & 55 & 56 & 0.2813 & 0.1433 & 0 & 0 & 1899 \\
\hline 56 & 56 & 57 & 1.59 & 0.5337 & 0 & 0 & 2200 \\
\hline 57 & 57 & 58 & 0.7837 & 0.263 & 0 & 0 & 2200 \\
\hline 58 & 58 & 59 & 0.3042 & 0.1006 & 100 & 72 & 1455 \\
\hline
\end{tabular}


TABle 4: Continued.

\begin{tabular}{|c|c|c|c|c|c|c|c|}
\hline \multirow[t]{2}{*}{ Line } & \multirow[t]{2}{*}{ Sending node } & \multirow[t]{2}{*}{ Receiving node } & \multirow[t]{2}{*}{ Resistance $(\Omega)$} & \multirow[t]{2}{*}{ Reactance $(\Omega)$} & \multicolumn{2}{|c|}{$\begin{array}{c}\text { Load at receiving } \\
\text { node }\end{array}$} & \multirow[t]{2}{*}{ Maximum line capacity (kVA) } \\
\hline & & & & & $P(\mathrm{~kW})$ & Q (kVAr) & \\
\hline 59 & 59 & 60 & 0.3861 & 0.1172 & 0 & 0 & 1455 \\
\hline 60 & 60 & 61 & 0.5075 & 0.2585 & 1244 & 888 & 1899 \\
\hline 61 & 61 & 62 & 0.0974 & 0.0496 & 32 & 23 & 1899 \\
\hline 62 & 62 & 63 & 0.145 & 0.0738 & 0 & 0 & 1899 \\
\hline 63 & 63 & 64 & 0.7105 & 0.3619 & 227 & 162 & 1899 \\
\hline 64 & 64 & 65 & 1.041 & 0.5302 & 59 & 42 & 1899 \\
\hline 65 & 11 & 66 & 0.2012 & 0.0611 & 18 & 13 & 1455 \\
\hline 66 & 66 & 67 & 0.0047 & 0.0014 & 18 & 13 & 1455 \\
\hline 67 & 12 & 68 & 0.7394 & 0.2444 & 28 & 20 & 1455 \\
\hline 68 & 68 & 69 & 0.0047 & 0.0016 & 28 & 20 & 1455 \\
\hline
\end{tabular}

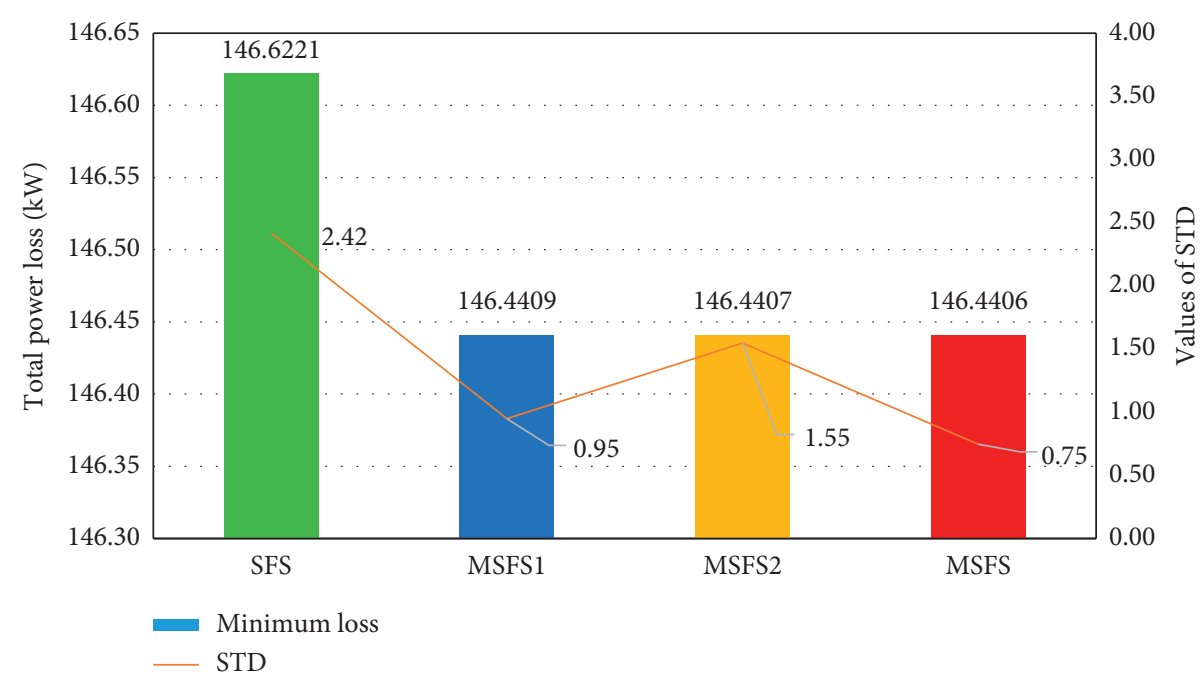

FIGURE 11: Minimum power loss and standard deviation of 50 trial runs obtained by SFS methods for case 1 of the 69-bus system.

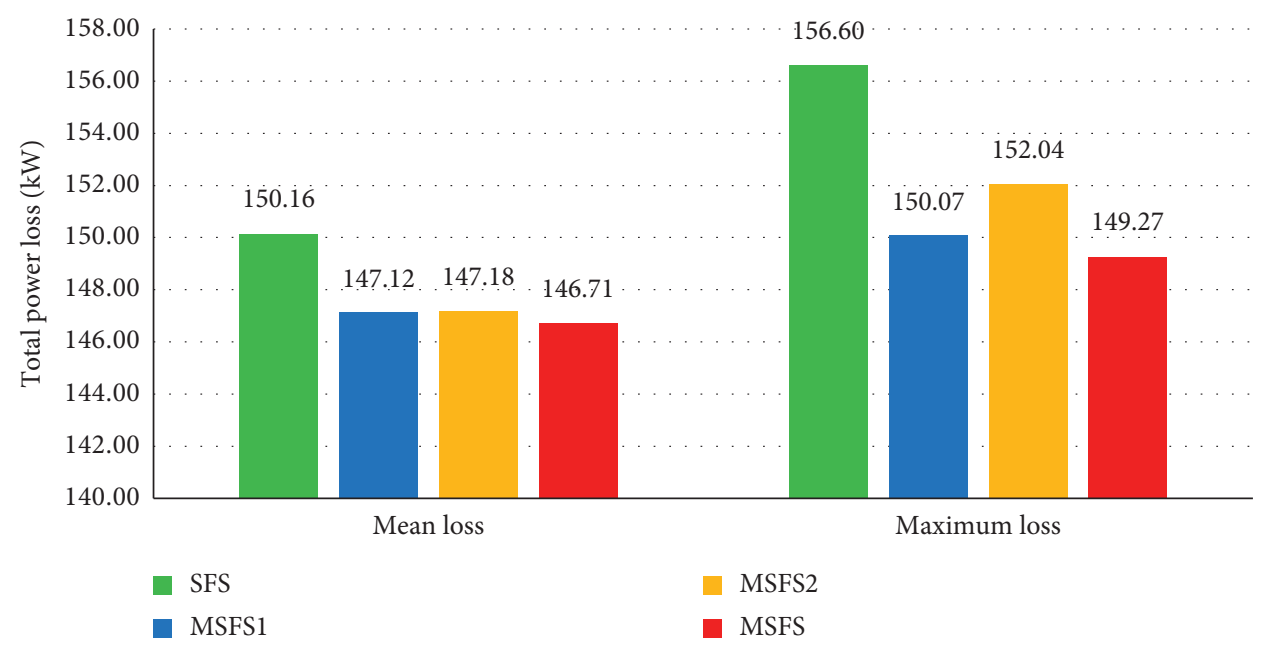

FIGURE 12: Mean and maximum power loss of 50 trial runs obtained by SFS methods for case 1 of the 69-bus system.

by $0.03,1.55$, and $2.21 \mathrm{~kW}$, respectively. The outstanding performance of MSFS over SFS can be clearly seen through Figure 21. The fluctuations of SFS are higher than MSFS1 and MSF2 and much higher than MSFS. MSFS not only has very smaller oscillations but also reaches many solutions with the same quality as the best solution. The manner can 


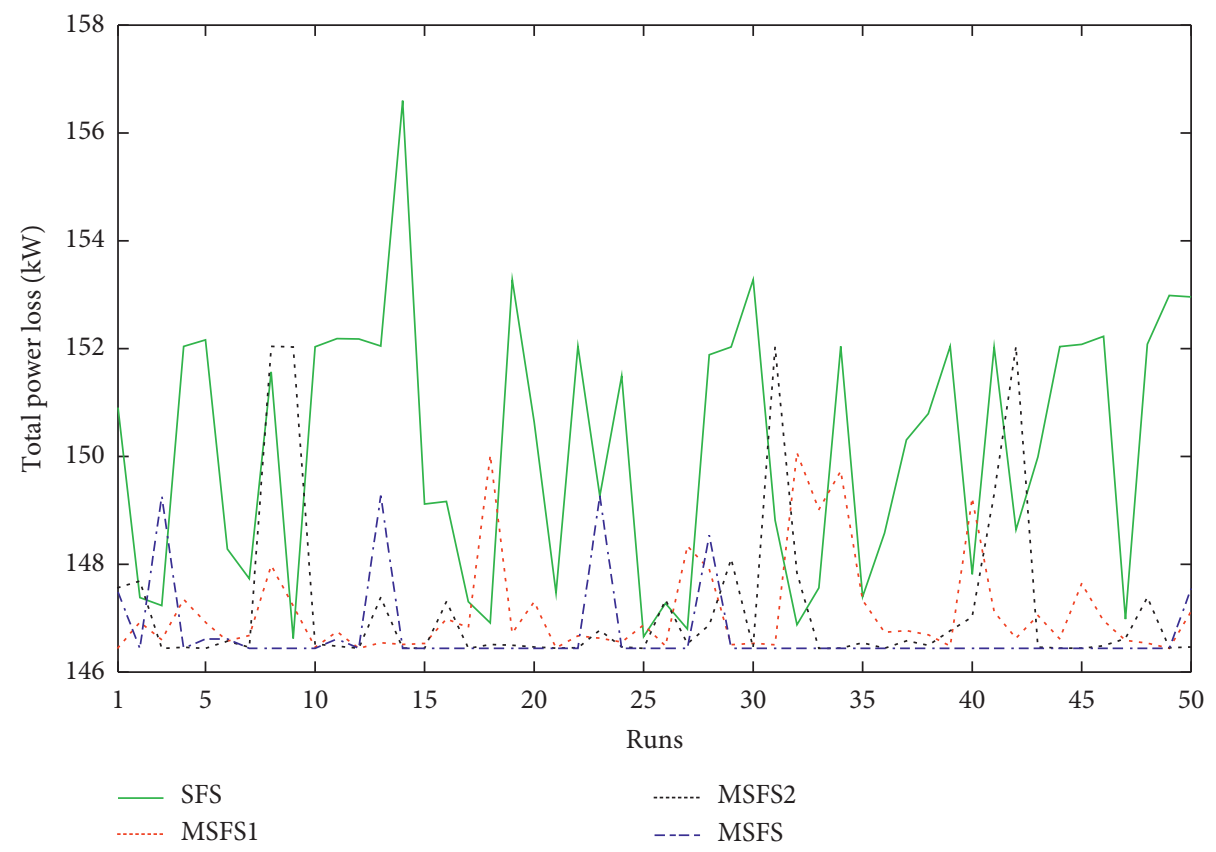

FIgURE 13: Total power loss of 50 trial runs obtained by SFS methods for case 1 of the 69-bus system.

TABle 5: Comparison with other methods for case 1 of the 69-bus distribution system.

\begin{tabular}{|c|c|c|c|c|c|c|c|c|c|c|}
\hline Method & $N_{\text {Po }}$ & $N_{\text {Iter }}$ & Bus & Size & $\begin{array}{c}\text { Total capacity } \\
(\mathrm{kVAr})\end{array}$ & Min. loss $(\mathrm{kW})$ & Mean loss $(\mathrm{kW})$ & Max. loss $(\mathrm{kW})$ & STD & $\mathrm{CT}(\mathrm{s})$ \\
\hline $\begin{array}{l}\text { Base network } \\
(\mathrm{Nc}=0)\end{array}$ & - & - & - & - & - & 225 & - & - & - & - \\
\hline PSO [17] & 30 & 1000 & $\begin{array}{l}61 \\
64\end{array}$ & $\begin{array}{c}1029 \\
207\end{array}$ & 1236 & 152.0541 & - & - & - & - \\
\hline RCGA [25] & 30 & 1000 & $\begin{array}{l}61 \\
64\end{array}$ & $\begin{array}{c}1029 \\
207\end{array}$ & 1236 & 152.0541 & - & - & - & - \\
\hline NCB-HM [42] & NR & NR & $\begin{array}{l}61 \\
17\end{array}$ & $\begin{array}{c}1224 \\
356\end{array}$ & 1580 & 146.5 & - & - & - & - \\
\hline HM [45] & NR & NR & $\begin{array}{l}61 \\
18\end{array}$ & $\begin{array}{c}1240 \\
350\end{array}$ & 1590 & 146.5 & - & - & - & - \\
\hline MSFS & 10 & 20 & $\begin{array}{l}17 \\
61\end{array}$ & $\begin{array}{c}360.94 \\
1275.06\end{array}$ & 1636 & 146.44 & 146.7059 & 149.2724 & 0.7467 & 0.84 \\
\hline
\end{tabular}

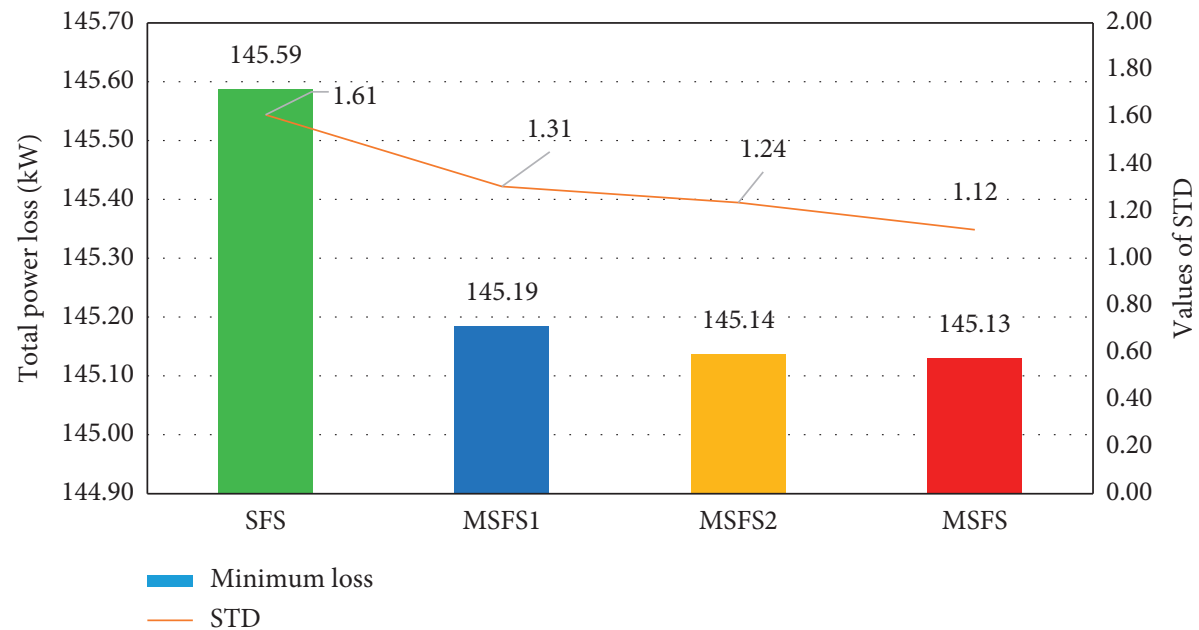

FIgURE 14: Minimum power loss and standard deviation of 50 trial runs obtained by SFS methods for case 2 of the 69-bus system. 


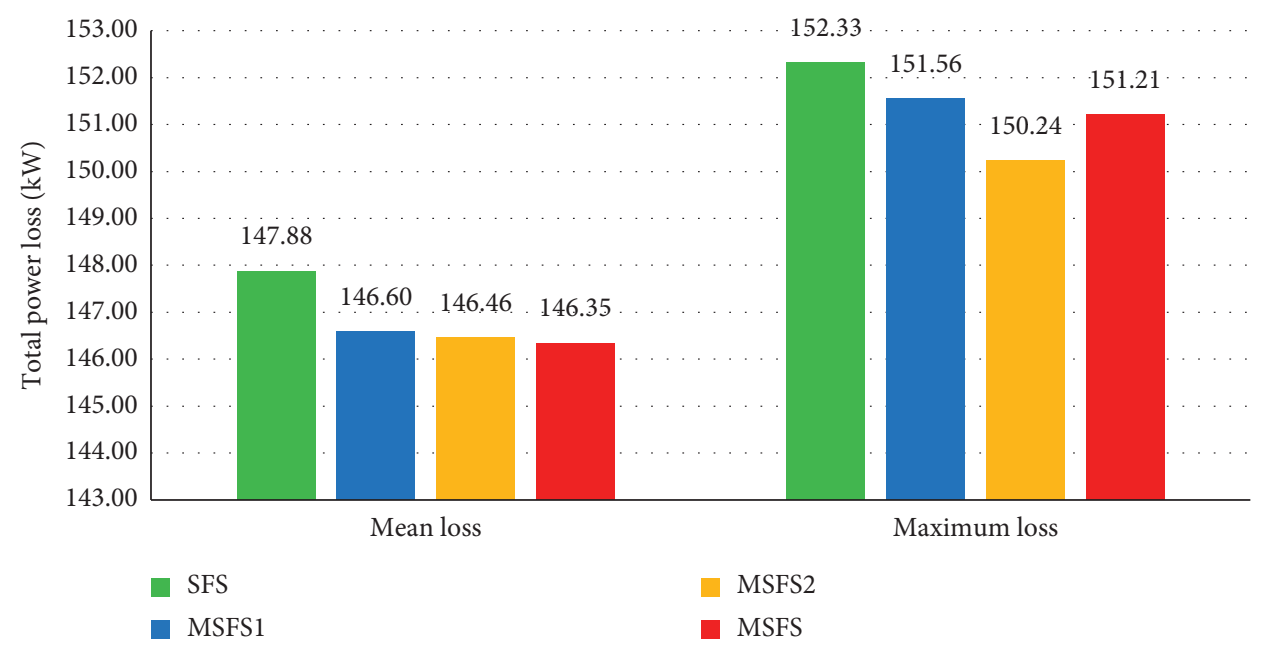

Figure 15: Mean and maximum power loss of 50 trial runs obtained by SFS methods for case 2 of the 69-bus system.

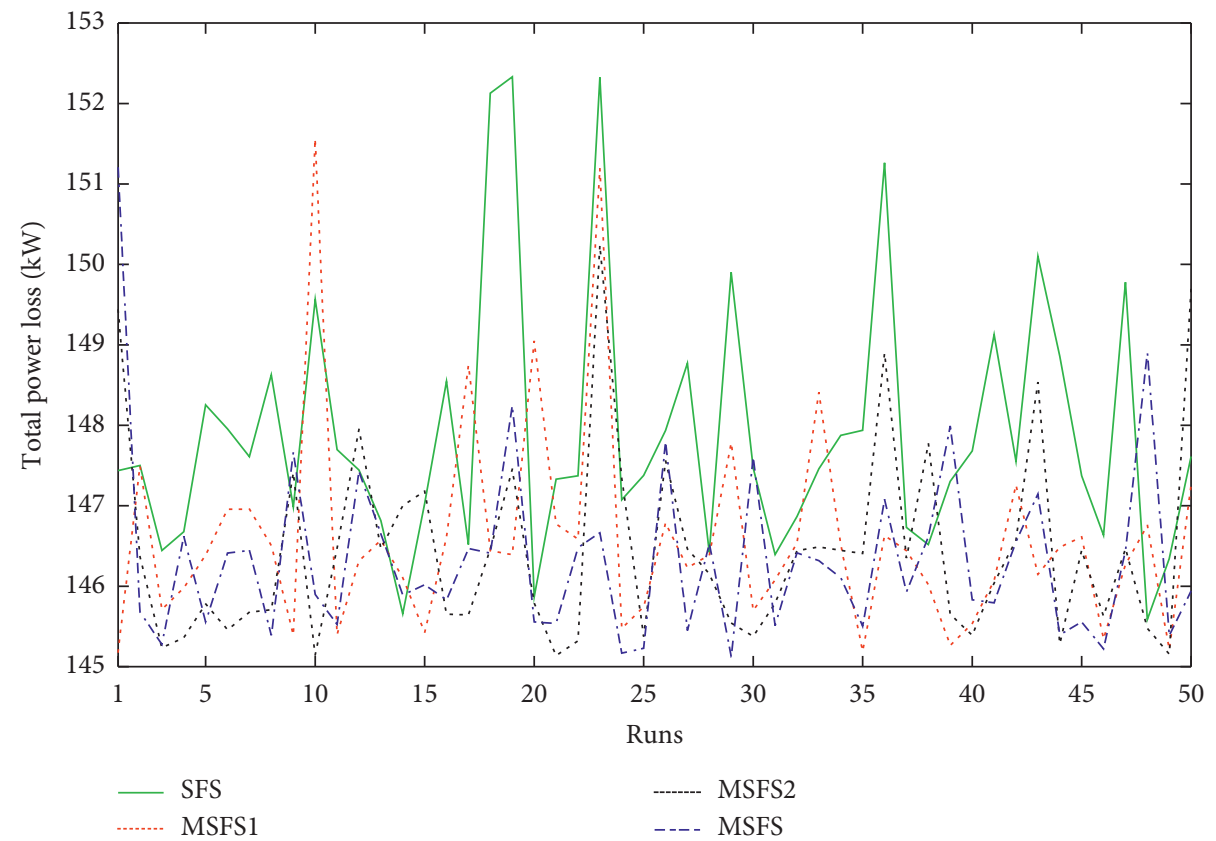

FIgURE 16: Total power loss of 50 trial runs obtained by SFS methods for case 2 of the 69-bus system.

confirm the real performance of the proposed method as compared to its conventional version.

However, the proposed method's performance cannot be further investigated for the case because previous studies have not considered the case of 2 capacitor banks for the system with 85 nodes. So, the performance investigation can be implemented for the next cases with 3, 4, and 14 capacitor banks.

5.3.2. Case 2: Three Capacitor Banks $(\mathrm{Nc}=3$ ). In this section, the proposed methods and other SFS methods are applied for the case with three capacitors by setting $N_{\mathrm{Df}}, N_{\mathrm{Po}}$, and $N_{\text {Iter }}$ to 2,10, and 25, respectively. Result comparisons for the case are shown in Figures 22-24. The first two figures have the same characteristics since SFS is the worst method with the highest values of loss and standard deviation while MSFS is the best method with the lowest values. The proposed method can reach less minimum loss, mean loss, and maximum loss than SFS by $1.88,4.62$, and $7.42 \mathrm{~kW}$, respectively. Figure 24 shows that all the fluctuations of the proposed method are much lower than those of SFS and approximately all points of the proposed method have lower loss than those of SFS. In the figure, it is hard to indicate a better method between MSFS1 and MSFS2, but the two methods are more effective than SFS. The proposed method finds three best solutions and many local optimal solutions with slightly less loss than these best solutions while SFS cannot converge to nearby global optimal solutions or the same local optimal solutions. Clearly, the new diffusion 
TABle 6: Comparison with other methods for case 2 of the 69-bus system.

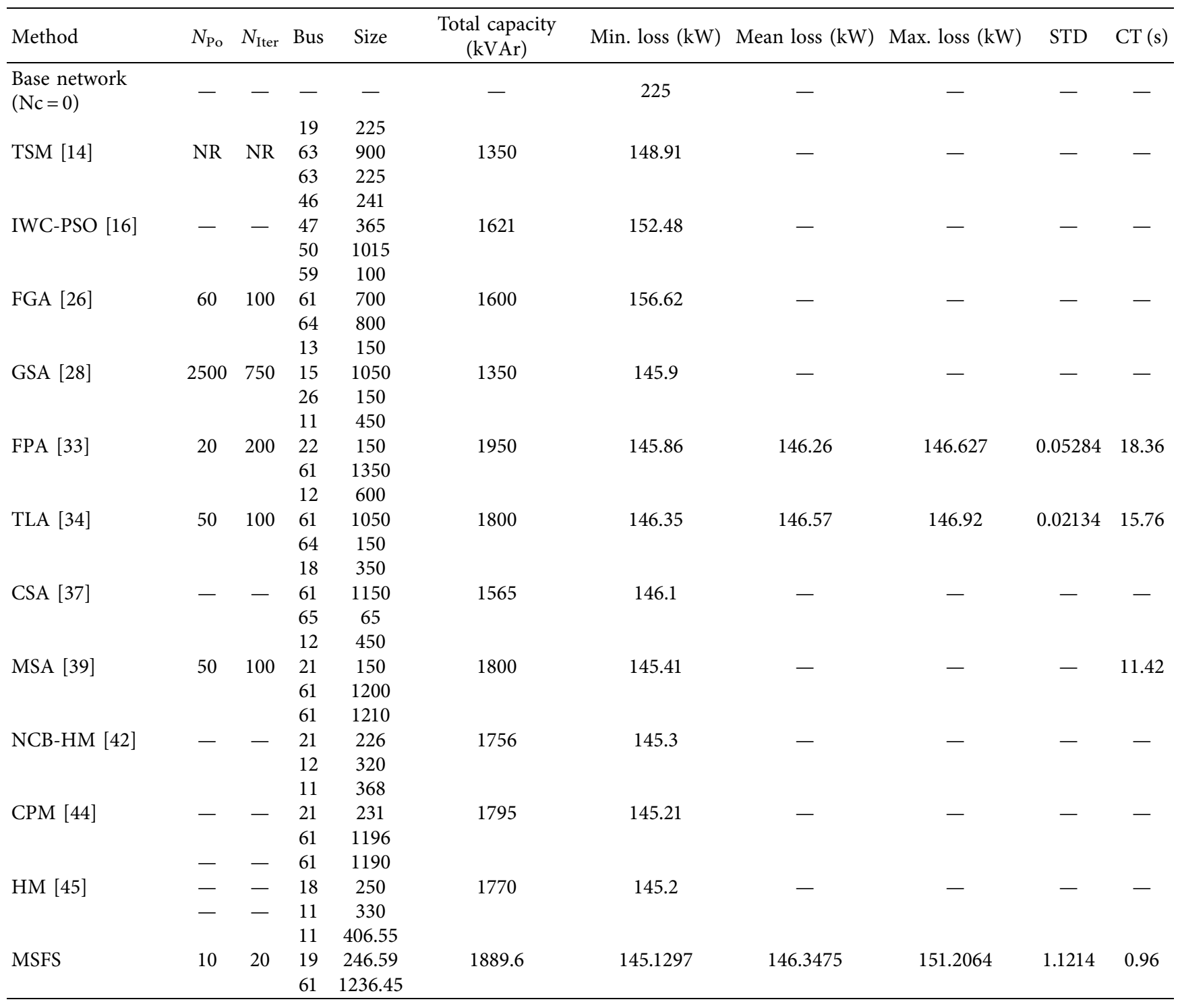

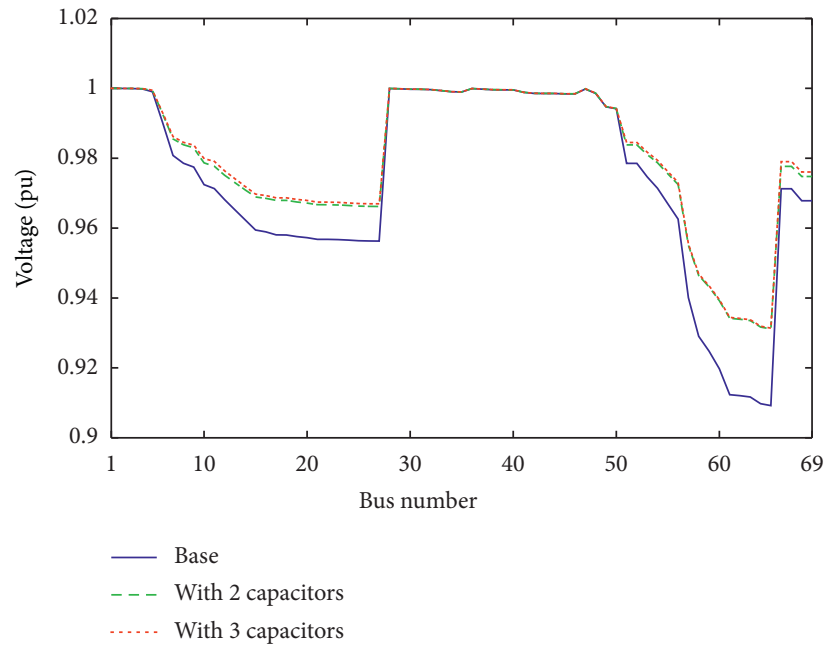

FIGURE 17: Voltage profile of the 69-bus network for different cases of capacitor placement. 


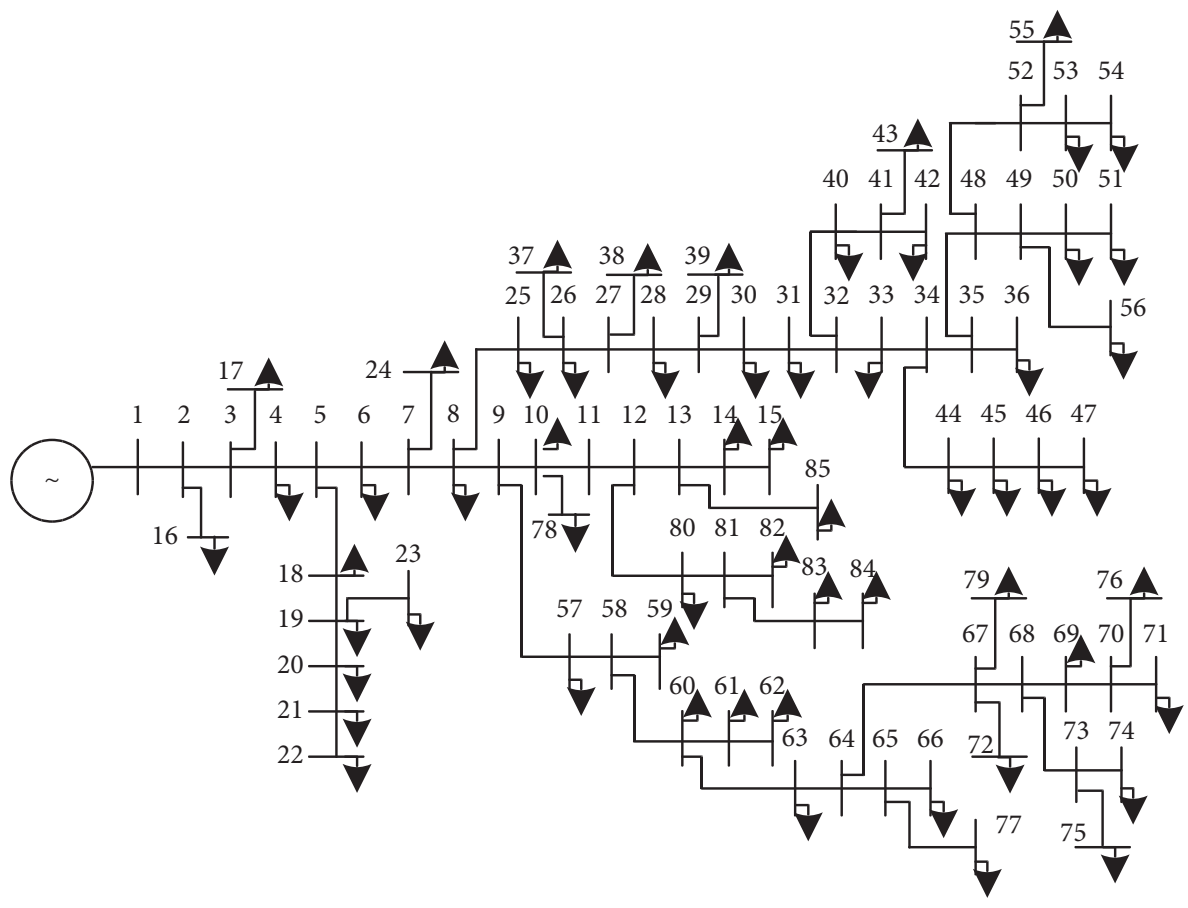

FIgURE 18: The 85-bus distribution system.

technique and two newly proposed update techniques are useful for the proposed method.

The comparison of result with other previous methods for the case is reported in Table 8 . The loss of the base network is $316.12 \mathrm{~kW}$ and that of the proposed method is $150.8555 \mathrm{~kW}$ while that of BFOA [31] and FPA [33] is 152.25 and $151.807 \mathrm{~kW}$. The loss indicates that the proposed method can reach less loss than the base network and these methods by $165.3445,1.3945$, and $0.9515 \mathrm{~kW}$, respectively. The loss reduction is, respectively, equivalent to $52.3 \%, 0,92 \%$, and $6.3 \%$, and these values are also better effectiveness levels of the proposed method. FPA reported better mean loss, maximum loss, and standard deviation, but it used much higher values for control parameters and took longer simulation time. The method has used 20 and 200 for population and iterations and spent 18.36 seconds for simulation time while these parameters are only 10 and 25 and simulation time is 2.5 seconds for the proposed method. Clearly, FPA could reach better stability thanks to the higher population size and more iterations. However, its best solution is worse than that of the proposed method and its search speed is slower. So, FPA cannot be a competitive method of the proposed method. BFOA [31] did not report mean loss, maximum loss, and calculation time for comparison. But the comparison with the method can easily to lead to a conclusion because it used higher population size and much more iterations and reached higher loss. So, the proposed method is superior to BFOA and FPA for the study case.

5.3.3. Case 3: Four Capacitor Banks $(N c=4)$. In this section, the number of capacitors is increased to 4, leading to the increase of control variables to 8,4 control variables for location, and 4 control variables for size of capacitors. So, the four methods are executed by setting $N_{\mathrm{Df}}, N_{\mathrm{Po}}$, and $N_{\text {Iter }}$ to 2 , 20 , and 30, respectively. The simulation results of the case are shown in Figures 25-27. The results of minimum loss, standard deviation, and mean loss have the same characteristic with the effective order of SFS, MSFS1, MSFS2, and MSFS. Figure 27 shows the highest loss of points from SFS and many points with lower loss from the proposed method. For the large study case, the proposed method can reach less loss than SFS by $2.03 \mathrm{~kW}$ equivalent to $1.35 \%$, and approximately all runs of the proposed method have less loss than those of SFS. Clearly, MSFS is still the best method with much better performance than SFS.

The comparison with MINPM [27] is shown in Table 9. The method only reported the minimum loss with $159.87 \mathrm{~kW}$, which is higher than $148.7077 \mathrm{~kW}$ of the proposed method by $11.1623 \mathrm{~kW}$. The less loss is corresponding to the improvement level of $6.98 \%$. Clearly, the proposed method is much more effective than MINPM [27]. The proposed method's simulation time is 7.7 seconds while that of MINPM [27] was not reported for comparison. The minimum loss can confirm the outstanding performance of the proposed method as compared to MINPM [27] for the study case.

5.3.4. Case 4: Fourteen Capacitor Banks $(N c=14)$. In this section, the most complicated case with fourteen capacitors is considered for testing the real performance of the proposed method and other ones. The number of control variables is 28 , which is much higher than all above study cases. So, the setting of $N_{\mathrm{Df}}, N_{\text {Po }}$, and $N_{\text {Iter }}$ is 2,20 , and 75 , respectively. Figures 28-30 show that the proposed method is the best method and SFS method is the worst method. Namely, the proposed method finds less loss than SFS by 
TABLE 7: Data of the third system with 85 buses.

\begin{tabular}{|c|c|c|c|c|c|c|}
\hline \multirow{2}{*}{ Line } & \multirow{2}{*}{ Sending node } & \multirow{2}{*}{ Receiving node } & \multirow{2}{*}{ Resistance $(\Omega)$} & \multirow{2}{*}{ Reactance $(\Omega)$} & \multicolumn{2}{|c|}{ Load at receiving node } \\
\hline & & & & & $P(\mathrm{~kW})$ & $Q(\mathrm{kVAr})$ \\
\hline 1 & 1 & 2 & 0.108 & 0.075 & 0 & 0 \\
\hline 2 & 2 & 3 & 0.163 & 0.112 & 0 & 0 \\
\hline 3 & 3 & 4 & 0.217 & 0.149 & 56 & 57.13 \\
\hline 4 & 4 & 5 & 0.108 & 0.074 & 0 & 0 \\
\hline 5 & 5 & 6 & 0.435 & 0.298 & 35.28 & 35.99 \\
\hline 6 & 6 & 7 & 0.272 & 0.186 & 0 & 0 \\
\hline 7 & 7 & 8 & 1.197 & 0.82 & 35.28 & 35.99 \\
\hline 8 & 8 & 9 & 0.108 & 0.074 & 0 & 0 \\
\hline 9 & 9 & 10 & 0.598 & 0.41 & 0 & 0 \\
\hline 10 & 10 & 11 & 0.544 & 0.373 & 56 & 57.13 \\
\hline 11 & 11 & 12 & 0.544 & 0.373 & 0 & 0 \\
\hline 12 & 12 & 13 & 0.598 & 0.41 & 0 & 0 \\
\hline 13 & 13 & 14 & 0.272 & 0.186 & 35.28 & 35.99 \\
\hline 14 & 14 & 15 & 0.326 & 0.223 & 35.28 & 35.99 \\
\hline 15 & 2 & 16 & 0.728 & 0.302 & 35.28 & 35.99 \\
\hline 16 & 3 & 17 & 0.455 & 0.189 & 112 & 114.26 \\
\hline 17 & 5 & 18 & 0.82 & 0.34 & 56 & 57.13 \\
\hline 18 & 18 & 19 & 0.637 & 0.264 & 56 & 57.13 \\
\hline 19 & 19 & 20 & 0.455 & 0.189 & 35.28 & 35.99 \\
\hline 20 & 20 & 21 & 0.819 & 0.34 & 35.28 & 35.99 \\
\hline 21 & 21 & 22 & 1.548 & 0.642 & 35.28 & 35.99 \\
\hline 22 & 19 & 23 & 0.182 & 0.075 & 56 & 57.13 \\
\hline 23 & 7 & 24 & 0.91 & 0.378 & 35.28 & 35.99 \\
\hline 24 & 8 & 25 & 0.455 & 0.189 & 35.28 & 35.99 \\
\hline 25 & 25 & 26 & 0.364 & 0.151 & 56 & 57.13 \\
\hline 26 & 26 & 27 & 0.546 & 0.226 & 0 & 0 \\
\hline 27 & 27 & 28 & 0.273 & 0.113 & 56 & 57.13 \\
\hline 28 & 28 & 29 & 0.546 & 0.226 & 0 & 0 \\
\hline 29 & 29 & 30 & 0.546 & 0.226 & 35.28 & 35.99 \\
\hline 30 & 30 & 31 & 0.273 & 0.113 & 35.28 & 35.99 \\
\hline 31 & 31 & 32 & 0.182 & 0.075 & 0 & 0 \\
\hline 32 & 32 & 33 & 0.182 & 0.075 & 14 & 14.28 \\
\hline 33 & 33 & 34 & 0.819 & 0.34 & 0 & 0 \\
\hline 34 & 34 & 35 & 0.637 & 0.264 & 0 & 0 \\
\hline 35 & 35 & 36 & 0.182 & 0.075 & 35.28 & 35.99 \\
\hline 36 & 26 & 37 & 0.364 & 0.151 & 56 & 57.13 \\
\hline 37 & 27 & 38 & 1.002 & 0.416 & 56 & 57.13 \\
\hline 38 & 29 & 39 & 0.546 & 0.226 & 56 & 57.13 \\
\hline 39 & 32 & 40 & 0.455 & 0.189 & 35.28 & 35.99 \\
\hline 40 & 40 & 41 & 1.002 & 0.416 & 0 & 0 \\
\hline 41 & 41 & 42 & 0.273 & 0.113 & 35.28 & 35.99 \\
\hline 42 & 41 & 43 & 0.455 & 0.189 & 35.28 & 35.99 \\
\hline 43 & 34 & 44 & 1.002 & 0.416 & 35.28 & 35.99 \\
\hline 44 & 44 & 45 & 0.911 & 0.378 & 35.28 & 35.99 \\
\hline 45 & 45 & 46 & 0.911 & 0.378 & 35.28 & 35.99 \\
\hline 46 & 46 & 47 & 0.546 & 0.226 & 14 & 14.28 \\
\hline 47 & 35 & 48 & 0.637 & 0.264 & 0 & 0 \\
\hline 48 & 48 & 49 & 0.182 & 0.075 & 0 & 0 \\
\hline 49 & 49 & 50 & 0.364 & 0.151 & 36.28 & 37.01 \\
\hline 50 & 50 & 51 & 0.455 & 0.189 & 56 & 57.13 \\
\hline 51 & 48 & 52 & 1.366 & 0.567 & 0 & 0 \\
\hline 52 & 52 & 53 & 0.455 & 0.189 & 35.28 & 35.99 \\
\hline 53 & 53 & 54 & 0.546 & 0.226 & 56 & 57.13 \\
\hline 54 & 52 & 55 & 0.546 & 0.226 & 56 & 57.13 \\
\hline 55 & 49 & 56 & 0.546 & 0.226 & 14 & 14.28 \\
\hline 56 & 9 & 57 & 0.273 & 0.113 & 56 & 57.13 \\
\hline 57 & 57 & 58 & 0.819 & 0.34 & 0 & 0 \\
\hline 58 & 58 & 59 & 0.182 & 0.075 & 56 & 57.13 \\
\hline 59 & 58 & 60 & 0.546 & 0.226 & 56 & 57.13 \\
\hline
\end{tabular}


TABLE 7: Continued.

\begin{tabular}{|c|c|c|c|c|c|c|}
\hline \multirow{2}{*}{ Line } & \multirow{2}{*}{ Sending node } & \multirow{2}{*}{ Receiving node } & \multirow{2}{*}{ Resistance $(\Omega)$} & \multirow{2}{*}{ Reactance $(\Omega)$} & \multicolumn{2}{|c|}{ Load at receiving node } \\
\hline & & & & & $P(\mathrm{~kW})$ & $Q(\mathrm{kVAr})$ \\
\hline 60 & 60 & 61 & 0.728 & 0.302 & 56 & 57.13 \\
\hline 61 & 61 & 62 & 1.002 & 0.415 & 56 & 57.13 \\
\hline 62 & 60 & 63 & 0.182 & 0.075 & 14 & 14.28 \\
\hline 63 & 63 & 64 & 0.728 & 0.302 & 0 & 0 \\
\hline 64 & 64 & 65 & 0.182 & 0.075 & 0 & 0 \\
\hline 65 & 65 & 66 & 0.182 & 0.075 & 56 & 57.13 \\
\hline 66 & 64 & 67 & 0.455 & 0.189 & 0 & 0 \\
\hline 67 & 67 & 68 & 0.91 & 0.378 & 0 & 0 \\
\hline 68 & 68 & 69 & 1.092 & 0.453 & 56 & 57.13 \\
\hline 69 & 69 & 70 & 0.455 & 0.189 & 0 & 0 \\
\hline 70 & 70 & 71 & 0.546 & 0.226 & 35.28 & 35.99 \\
\hline 71 & 67 & 72 & 0.182 & 0.075 & 56 & 57.13 \\
\hline 72 & 68 & 73 & 1.184 & 0.491 & 0 & 0 \\
\hline 73 & 73 & 74 & 0.273 & 0.113 & 56 & 57.13 \\
\hline 74 & 73 & 75 & 1.002 & 0.416 & 35.28 & 35.99 \\
\hline 75 & 70 & 76 & 0.546 & 0.226 & 56 & 57.13 \\
\hline 76 & 65 & 77 & 0.091 & 0.037 & 14 & 14.28 \\
\hline 77 & 10 & 78 & 0.637 & 0.264 & 56 & 57.13 \\
\hline 78 & 67 & 79 & 0.546 & 0.226 & 35.28 & 35.99 \\
\hline 79 & 12 & 80 & 0.728 & 0.302 & 56 & 57.13 \\
\hline 80 & 80 & 81 & 0.364 & 0.151 & 0 & 0 \\
\hline 81 & 81 & 82 & 0.091 & 0.037 & 56 & 57.13 \\
\hline 82 & 81 & 83 & 1.092 & 0.453 & 35.28 & 35.99 \\
\hline 83 & 83 & 84 & 1.002 & 0.416 & 14 & 14.28 \\
\hline 84 & 13 & 85 & 0.819 & 0.34 & 35.28 & 35.99 \\
\hline
\end{tabular}

$2.36 \mathrm{~kW}$ for minimum loss, $4.3 \mathrm{~kW}$ for mean loss, and $5.63 \mathrm{~kW}$ for maximum loss. The improvement level of the proposed method over SFS is $1.63 \%$ for the study case.

For the study case, the comparison with other methods is shown in Table 10. The proposed method can reach less minimum loss and shorter simulation time than TLA [34]. The two factors are $142.4999 \mathrm{~kW}$ and 15.72 seconds for the proposed method and $143.18 \mathrm{~kW}$ and 18.38 seconds for TLA [34]. The proposed method can reduce power loss more effectively than TLA by $0.68 \mathrm{~kW}$ corresponding to $0.47 \%$. For other results such as mean loss, maximum loss, and standard deviation, TLA is more effective; however, it should be noted that TLA was run by setting 50 and 100 to population and iteration while the values of the proposed method are 20 and 75 . The stability of search from TLA is better because it used higher control parameters, but the best solution of TLA is worse than that of the proposed method. So, it can be concluded that the proposed method is very effective for the study case.

Figure 31 shows the improvement of voltage profile after installing different number of capacitors in the 85-bus distribution system. The voltage profile of the base system without capacitors in blue is the worst among five cases of voltage. Voltage profile of four other cases is much better than that of the base network. The lowest voltage of base network is about less than 0.88 pu but that of other cases is higher than $0.92 \mathrm{pu}$. Furthermore, the voltage profile from node 25 to node 85 in the base network without capacitors is under $0.9 \mathrm{pu}$, but it is higher than $0.92 \mathrm{pu}$ in the modified network with capacitors. The comparisons between the case without capacitors and other cases with capacitors reveal that the voltage profile is not much improved when installing capacitors. However, the voltage of the case with 3 and 4 capacitors is not much better than that of the case with 2 capacitors. Similarly, voltage profile of network with 14 capacitors is not much better than that of the case with 3 and 4 capacitors. This manner can be understood easily because the purpose of the capacitor placement in the paper is for power loss reduction rather than for voltage profile improvement.

5.4. Result Comparison for the 69-Bus Distribution Network with the Objective Function of Total Cost. In this section, the total cost of energy loss over a year and capacitor investment is considered as objective function as shown in equation (2). The number of load levels, $M$, is three in which the number of hours in the first load level (called light load), in the second load level (called normal load), and the third load level (called peak load) is, respectively, 2000, 5260, and 1500 hours. The light load, normal load, and peak load are corresponding to $50 \%, 100 \%$, and $160 \%$ of the current loads in the 69 -bus distribution network [46]. The main difference of the total cost objective function and the power loss objective function is the size of capacitors. The size is a continuous variable in the previous section, but it is a discrete variable in this section. The size of capacitors is selected from 150 to 4050 with a step size of $150 \mathrm{kVAr}$. The price of each $\mathrm{kWh}$ and each $\mathrm{kVAr}$ is $\$ 0.06$ and $\$ 3.0$, respectively [46]. The data of the system are also taken from Table 4. 


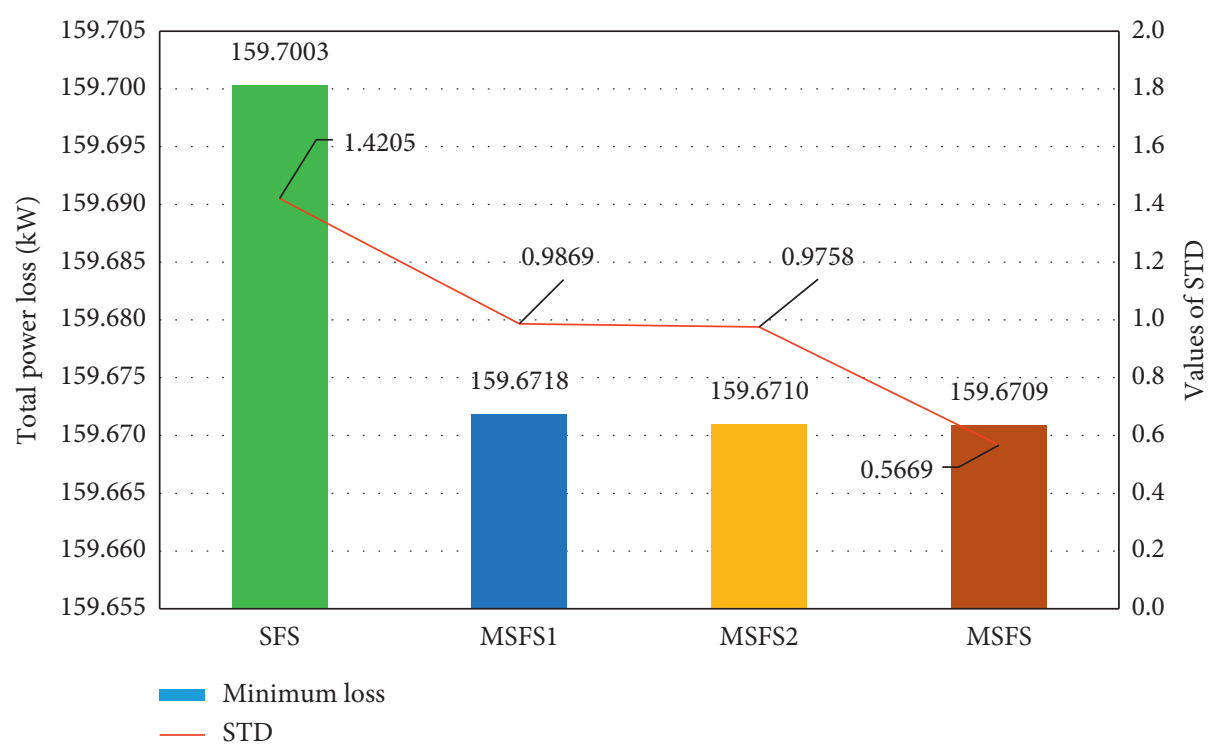

Figure 19: Minimum power loss and standard deviation of 50 trial runs obtained by SFS methods for case 1 of the 85 -bus system.

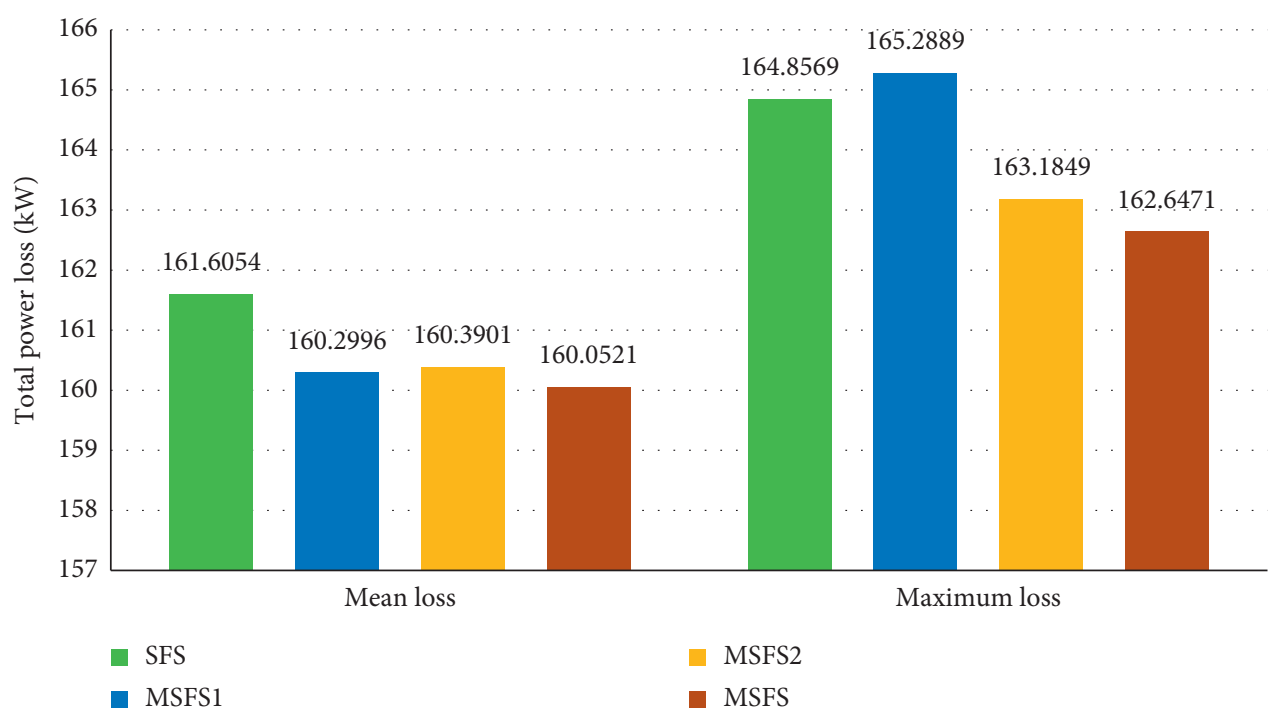

FIGURE 20: Mean and maximum power loss of 50 trial runs obtained by SFS methods for case 1 of the 85-bus system.

For reaching the total cost of the 69-bus system with the placement of three capacitors, SFS methods are run 50 independent trials by setting the number of diffusions, the size of population, and the number of iterations to 2,10 , and 30. The results obtained by the four SFS methods are reported in Table 11. The table can show the best performance of the proposed method since it finds the most optimal solution for placing three capacitors in the system. It gets the lowest minimum cost, but SFS suffers the highest cost. MSFS has achieved a lower cost than SFS about \$25.9, corresponding to $0.03 \%$ of the cost of SFS.

The results from the proposed method are compared to those of TLA [34] and DSOA [46], which are shown in Table 12 . In addition, location and size of each capacitor, power loss of the whole system corresponding to three load statuses including light load (LL), normal load (NL), and peak load $(\mathrm{PL})$, and total compensation reactive power are also reported in the table. As shown in the total compensation capacity row, MSFS and TLA [34] use the same $2100 \mathrm{kVAr}$ and much less than $3600 \mathrm{kVAr}$ of DOSA. So, the proposed method and TLA [34] expend the same capacitor investment cost of $\$ 6300$ while DSOA uses much higher capacitor cost, which is $\$ 10,800$ and higher than that of MSFS and DSOA [46] by $\$ 4500$. The power loss of the light load, nominal load, and peak load is (35.52, 147, 427.3) kW for TLA [34], (34.43, 146.8, $417.28) \mathrm{kW}$ for DSOA [46], and (34.228, 145.870, 419.016) kW for the proposed method. By using the 


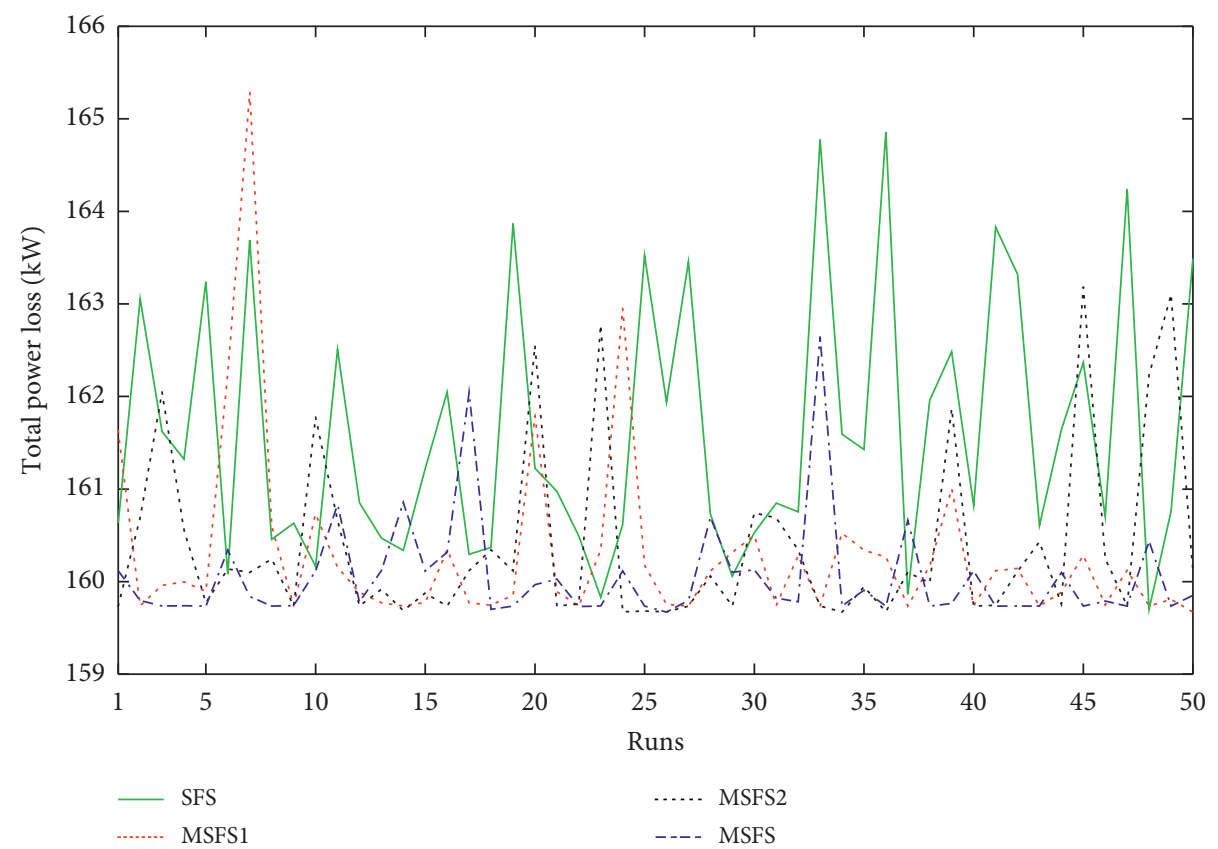

FIgURE 21: Total power loss of 50 trial runs obtained by SFS methods for case 1 of the 85-bus system.

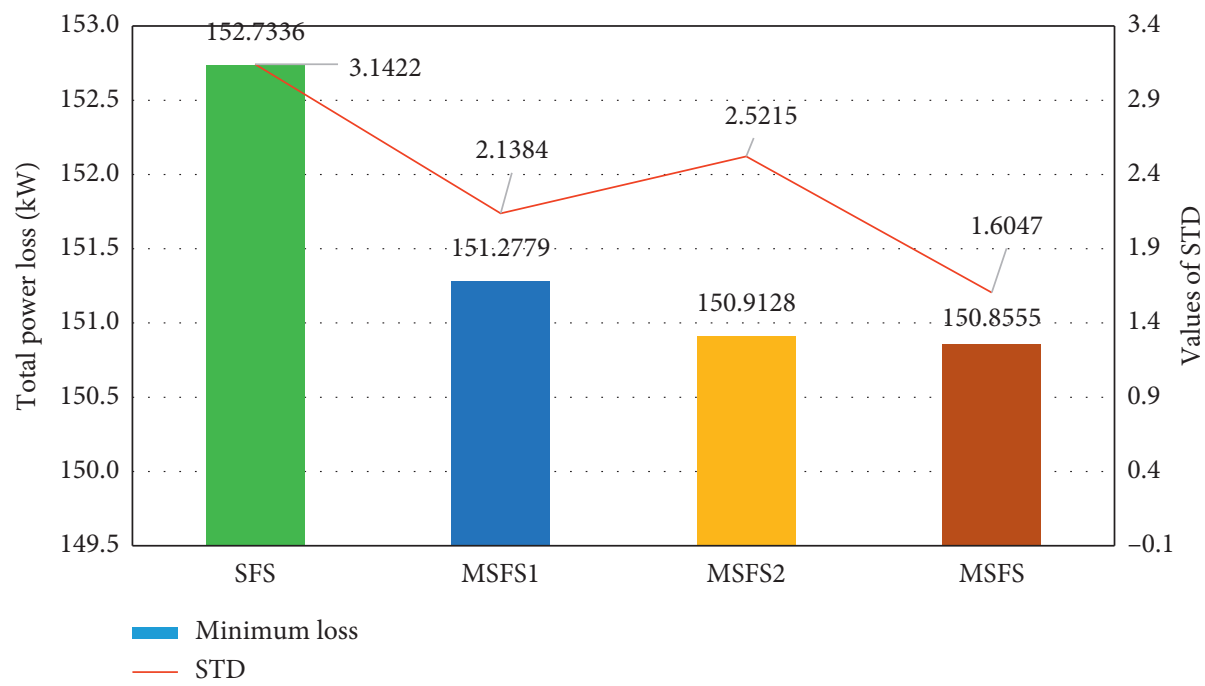

FIGURE 22: Minimum power loss and standard deviation of 50 trial runs obtained by SFS methods for case 2 of the 85 -bus system.

power loss, price of each $\mathrm{kWh}$, and the number of hours, the energy loss cost can be obtained for the three methods, and it is equal to $\$ 89,016.38$ for TLA, $\$ 89,112.60$ for DSOA [46] and $\$ 87,855.44$ for MSFS. By employing equation (2), total cost is calculated and reported for evaluating the real performance of the proposed method. As a result, the proposed MSFS is the best method with the lowest total cost of $\$ 94,155.43$ while that of TLA and DSOA is much higher and equal to $\$ 95,316.38$ and $\$ 99,912.60$. Clearly, the selection of capacitor can support the proposed method save $\$ 1,160.95$ and $\$ 5,757.17$ as compared to TLA and DSOA. The saving cost is corresponding to $1.22 \%$ and $5.76 \%$ of the total cost from TLA and DSOA. The saving cost and the improvement level are significant when implementing the proposed MSFS for the purpose of placing capacitors in the 69-bus distribution network instead of using TLA and DSOA. Furthermore, the proposed method can find more effective solutions much faster than TLA. In fact, $N_{\text {Po }}$ and $N_{\text {Iter }}$ are set to 50 and 100 for TLA but only 10 and 30 for the proposed MSFS method.

5.5. Result Comparison for the 85-Bus Distribution Network with the Objective Function of Total Cost. In this section, capacitors are added in the 85-bus distribution network with the purpose of minimizing the total cost of power loss and capacitor investment. All data of the system are reported in 


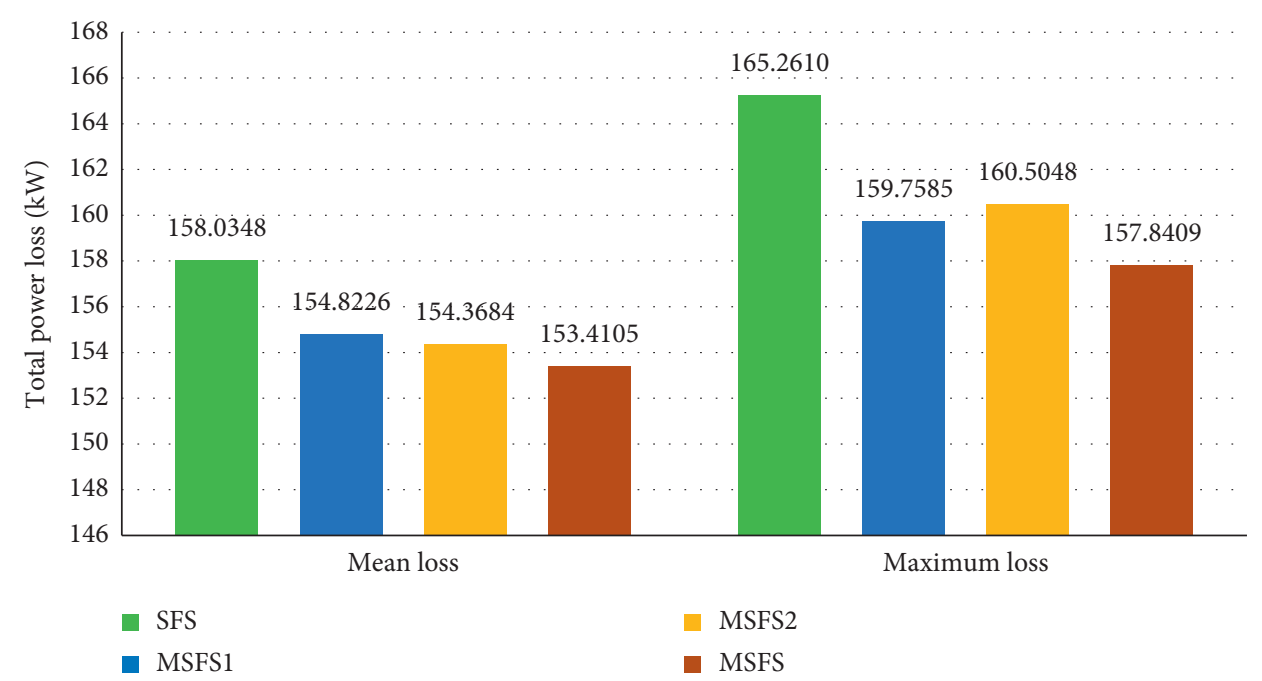

FIGURE 23: Mean and maximum power loss of 50 trial runs obtained by SFS methods for case 2 of the 85-bus system.

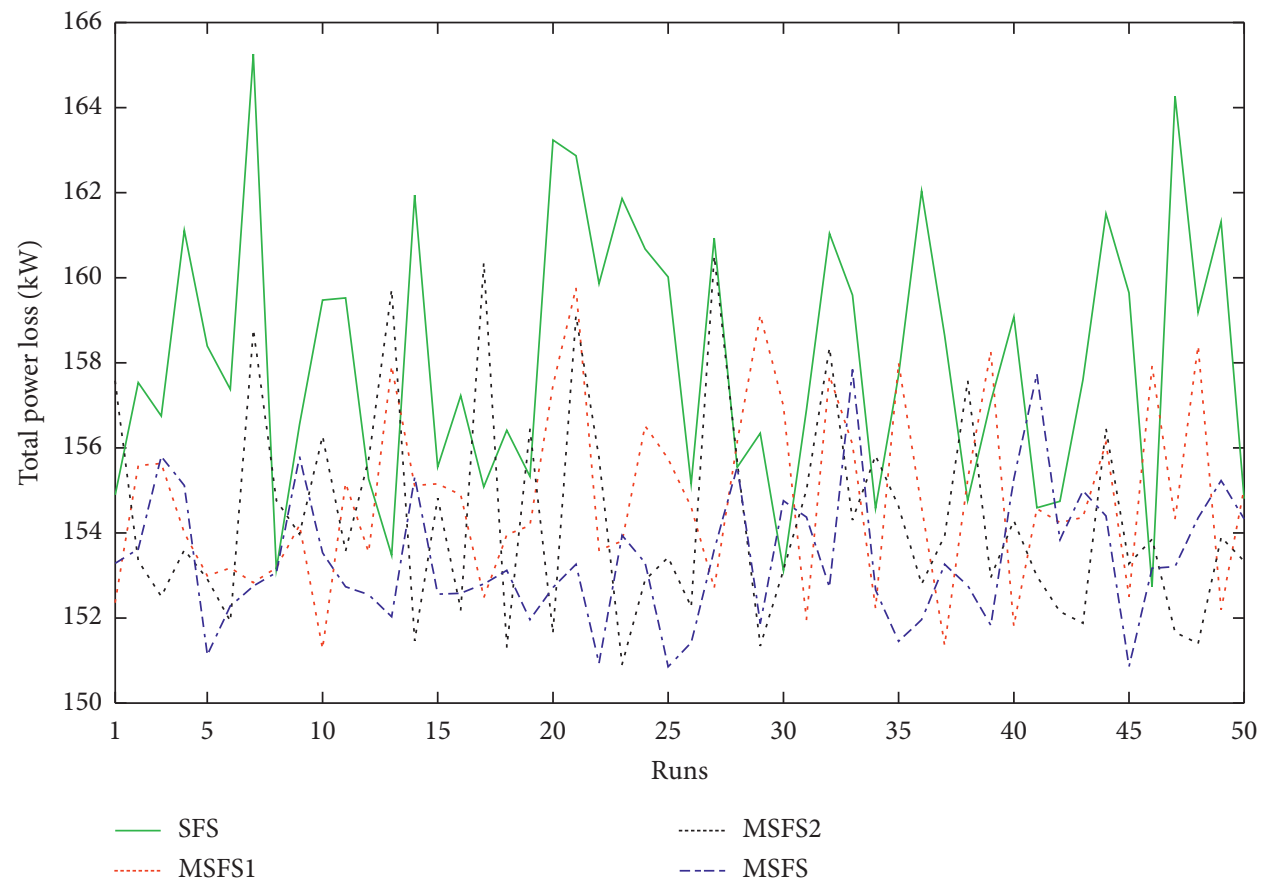

Figure 24: Total power loss of 50 trial runs obtained by SFS methods for case 2 of the 85-bus system.

Table 7 while other information such as price of energy, price of capacitor, time of each load status, and load levels is the same as that of the 69-bus distribution network. Summary of total cost from 50 independent trial runs by implementing four SFS methods is reported in Table 13. MSFS is still the best method, and SFS is always the worst method with the highest minimum, mean, and maximum cost. The proposed method can find less cost than SFS by $\$ 2,286.24$, which is corresponding to $2.31 \%$ of the total cost from SFS. The improvement level of the system is $2.31 \%$ while that of the 69 -bus distribution network is only $0.03 \%$. Obviously, the outstanding performance of the proposed method is much clearer for larger scale system.
Results from the proposed method and two compared methods such as TLA [34] and DSOA [46] are presented in Table 14. Location and size of each capacitor corresponding to LL, NL, and PL are optimal solutions, and they can support to calculate power loss for the three load statuses. The power loss is $(34.11,143.2493,411.6) \mathrm{kW}$ for TLA [34], (34.76 144.01, 410.69) kW for DSOA [46], and (34.943, $144.871,415.389) \mathrm{kW}$ for the proposed method. By using the power loss, the time of each load level, and the price of energy, the energy loss cost of TLA, DSOA, and the proposed can be obtained, and it is equal to $\$ 86,346.679$, $\$ 86,586$, and $\$ 87,299.544$, respectively. Although the energy loss cost of the proposed method is higher than that of 
TABLE 8: Comparison with other methods for case 2 of the 85-bus system.

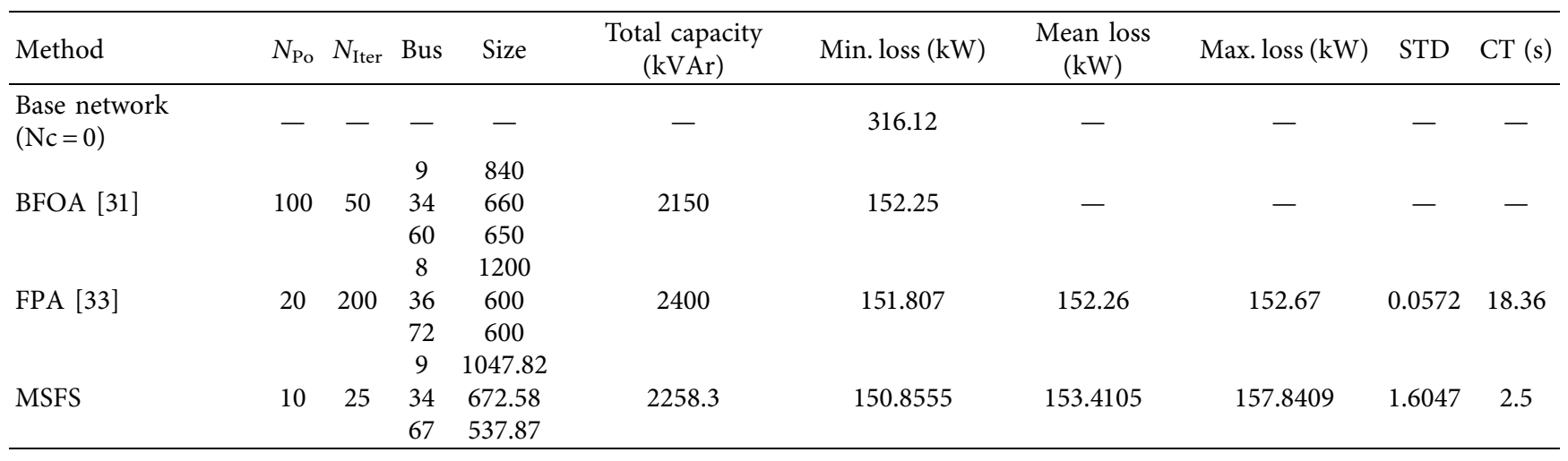

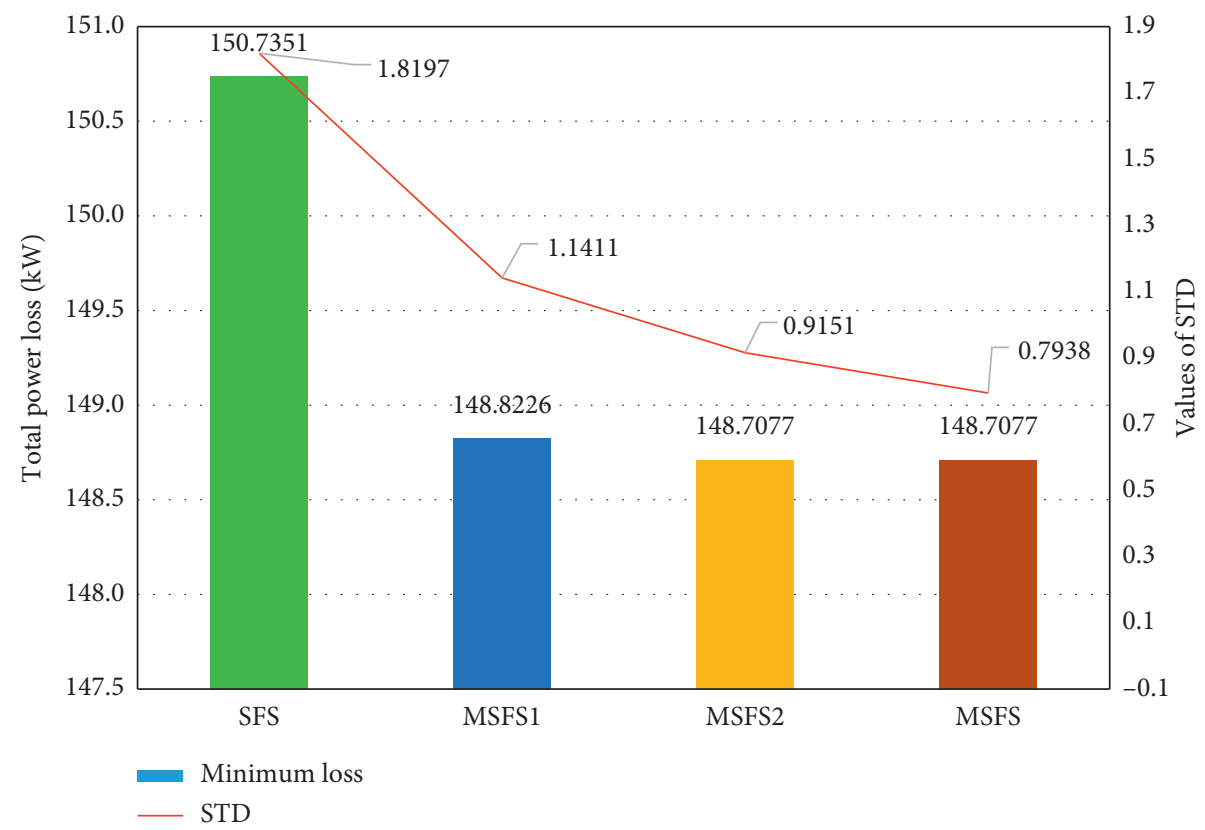

FIgURE 25: Minimum power loss and standard deviation of 50 trial runs obtained by SFS methods for case 3 of the 85-bus system.

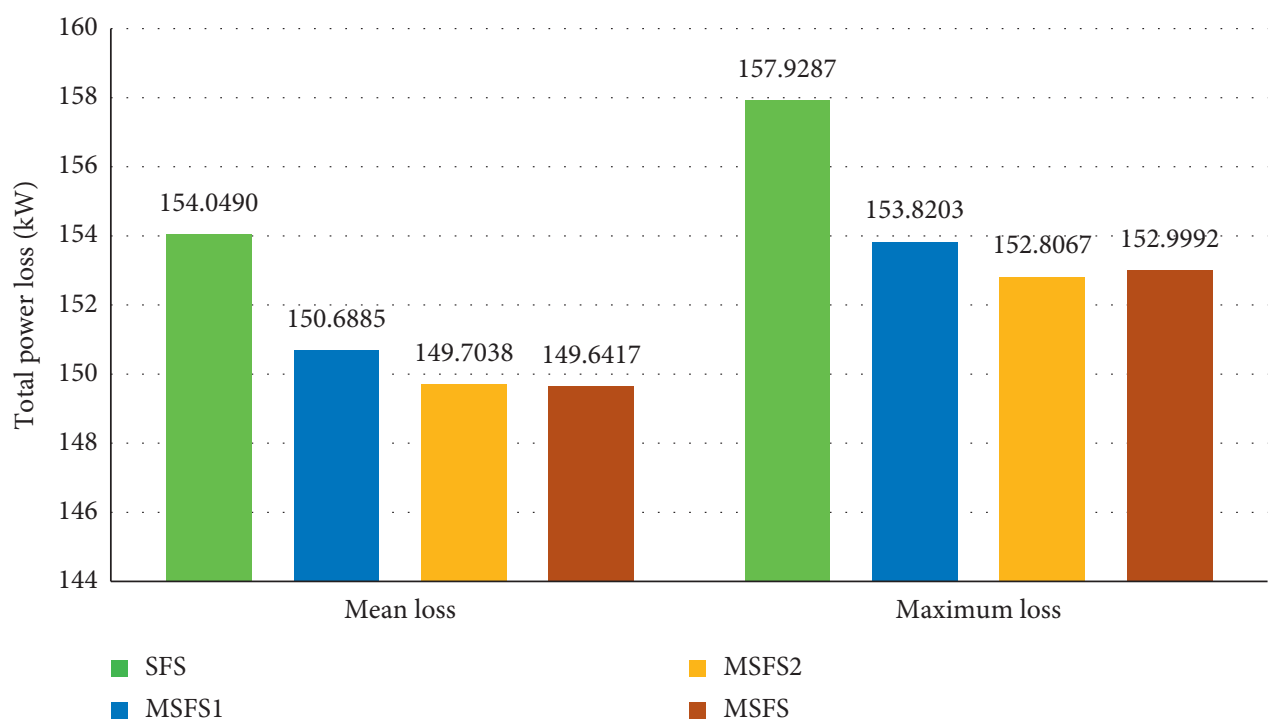

FIGURE 26: Mean and maximum power loss of 50 trial runs obtained by SFS methods for case 3 of the 85-bus system. 


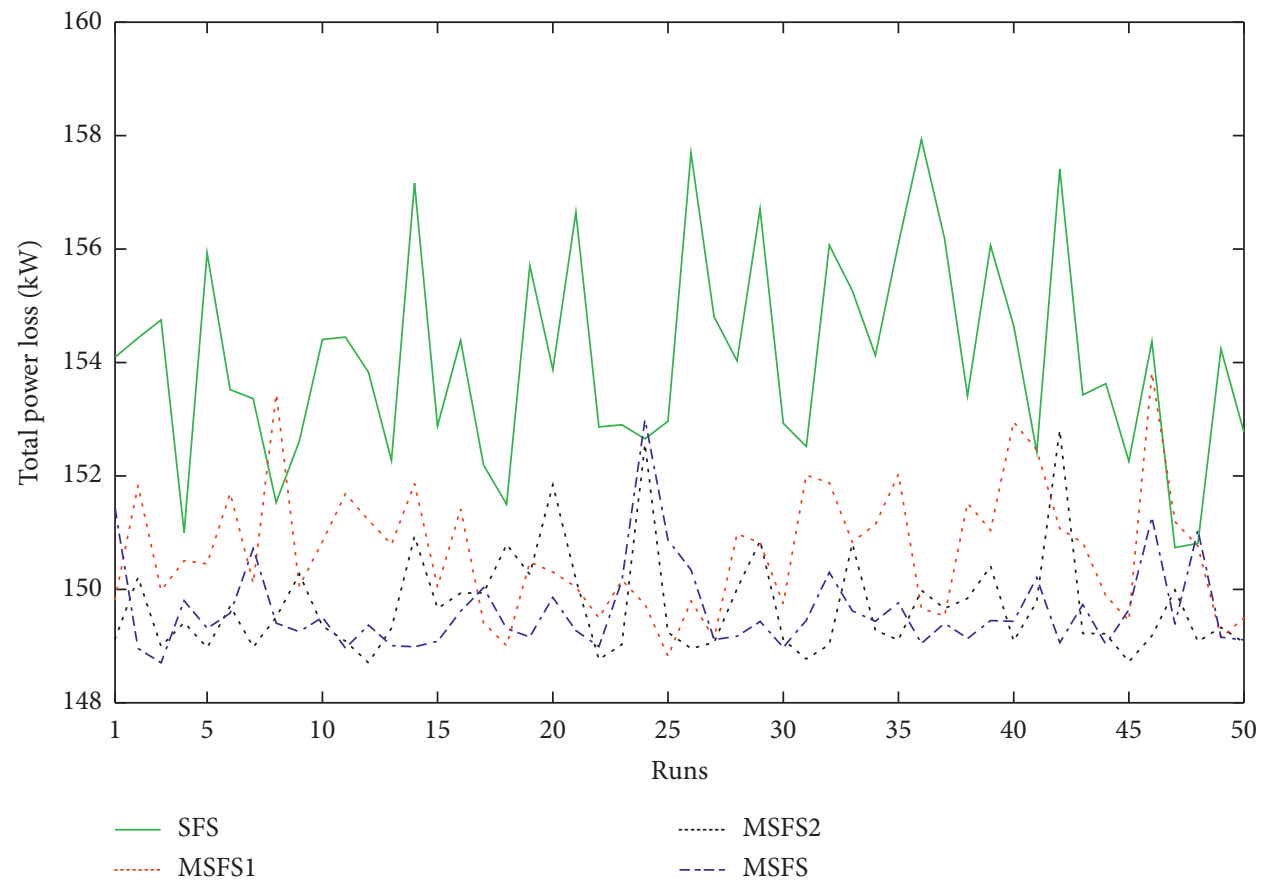

Figure 27: Total power loss of 50 trial runs obtained by SFS methods for case 3 of the 85-bus system.

TABLE 9: Comparison with other methods for case 3 of the 85-bus system.

\begin{tabular}{|c|c|c|c|c|c|c|c|c|c|c|}
\hline Method & $N_{\text {Po }}$ & $N_{\text {Iter }}$ & Bus & Size & $\begin{array}{c}\text { Total capacity } \\
\text { (kVAr) }\end{array}$ & Min. loss $(\mathrm{kW})$ & Mean loss $(\mathrm{kW})$ & Max. loss (kW) & STD & $\mathrm{CT}(\mathrm{s})$ \\
\hline $\begin{array}{l}\text { Base network } \\
(\mathrm{Nc}=0)\end{array}$ & - & - & - & - & - & 316.12 & - & - & - & - \\
\hline MINPM [27] & - & - & $\begin{array}{c}7 \\
8 \\
29 \\
58\end{array}$ & $\begin{array}{l}300 \\
700 \\
900 \\
500\end{array}$ & 2400 & 159.87 & - & - & - & - \\
\hline MSFS & 20 & 30 & $\begin{array}{c}8 \\
12 \\
34 \\
67\end{array}$ & $\begin{array}{c}758.24 \\
345.21 \\
660.1 \\
534.37\end{array}$ & 2297.9 & 148.7077 & 149.6417 & 152.9992 & 0.7938 & 7.7 \\
\hline
\end{tabular}

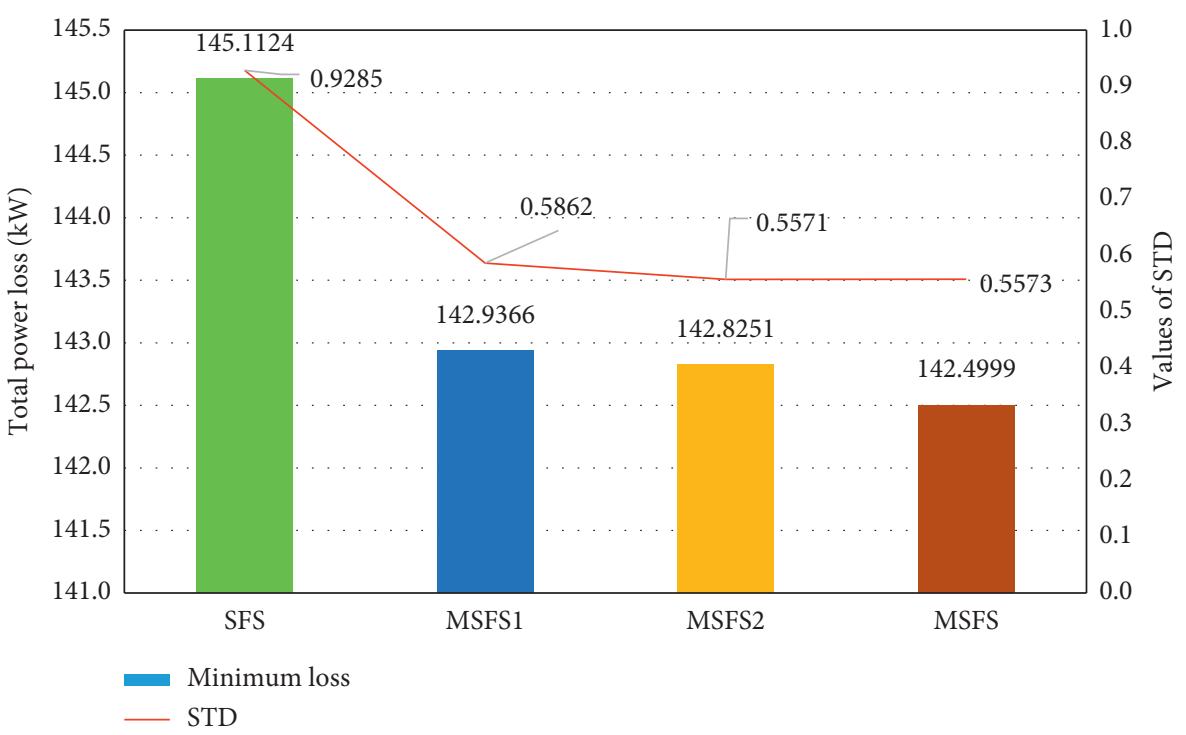

Figure 28: Minimum power loss and standard deviation of 50 trial runs obtained by SFS methods for case 4 of the 85-bus system. 


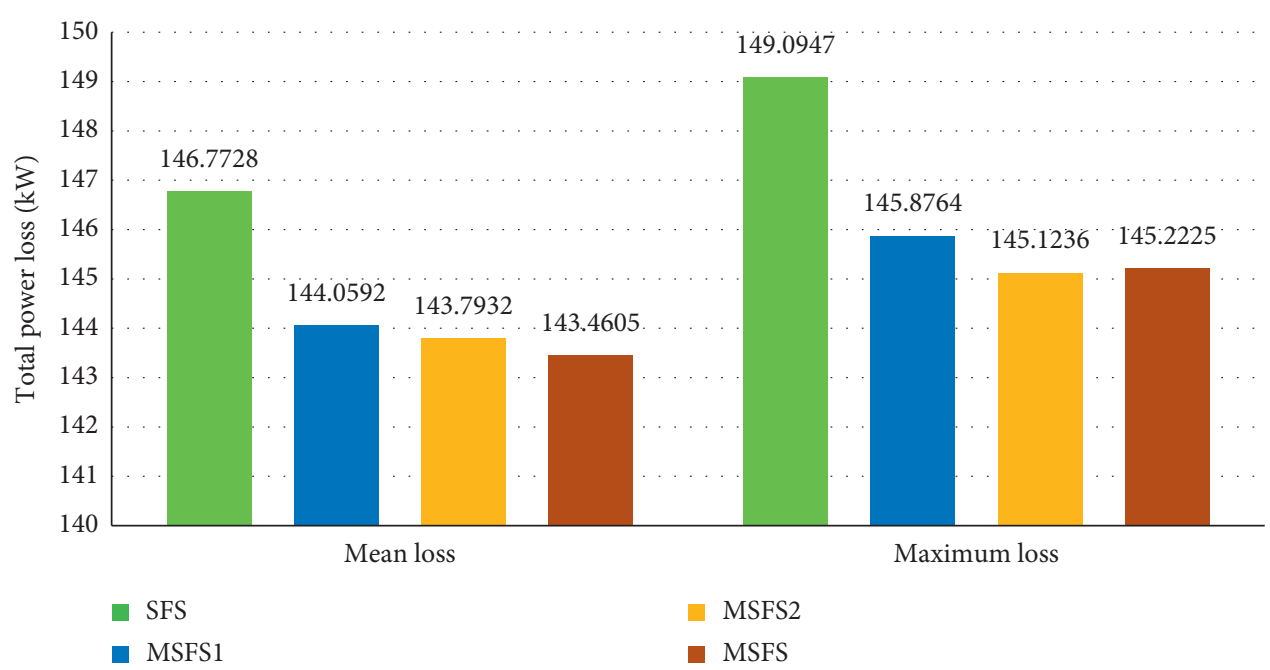

Figure 29: Mean and maximum power loss of 50 trial runs obtained by SFS methods for case 4 of the 85-bus system.

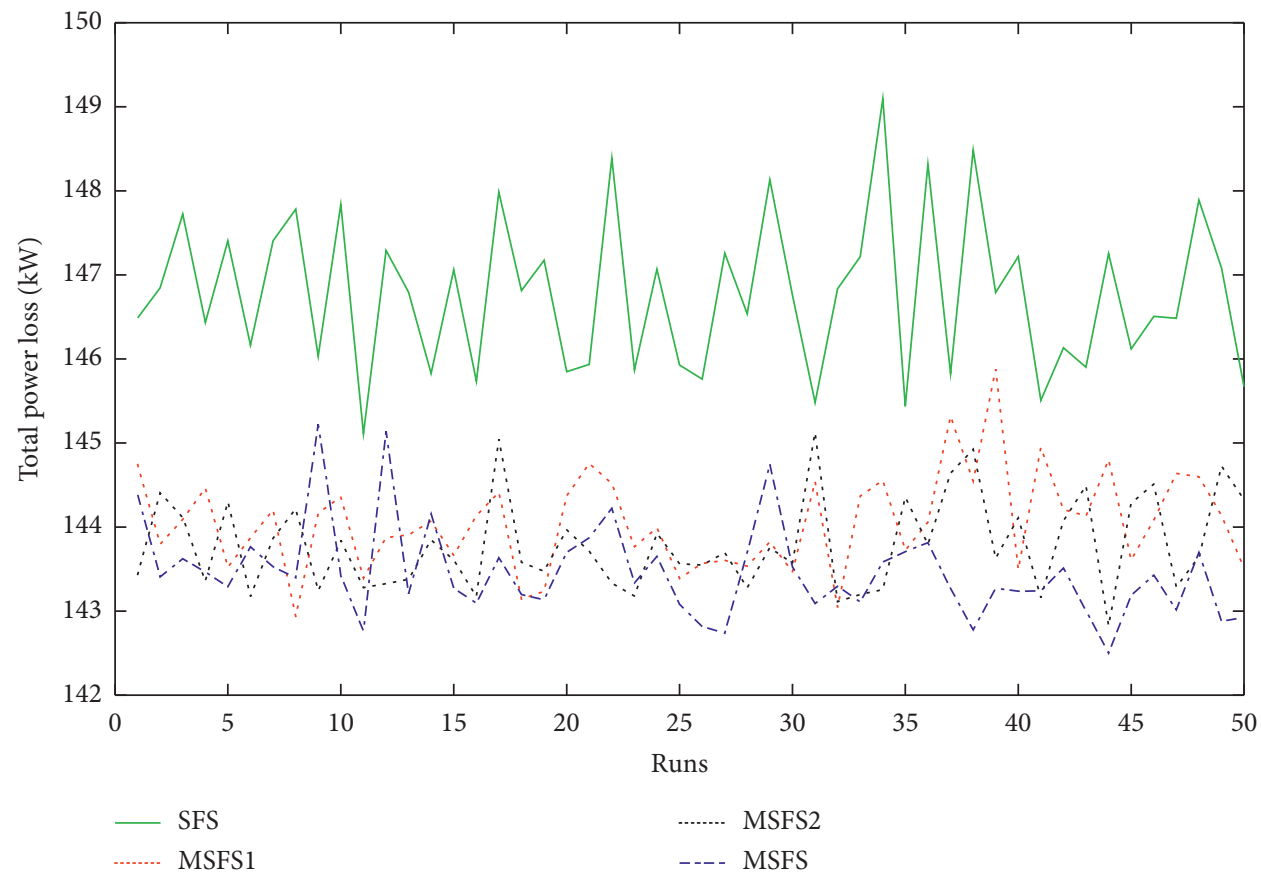

FIgURe 30: Total power loss of 50 trial runs obtained by SFS methods for case 4 of the 85-bus system.

TLA and DSOA, it uses smaller total capacity. In fact, the total compensation of the proposed method is only $3150 \mathrm{kVAr}$ but TLA and DSOA use 3750 and $5650 \mathrm{kVAr}$. So, the capacitor investment of the proposed method is the lowest, $\$ 9450$, but that of TLA and DSOA is $\$ 11,250$ and $\$ 13,950$. By adding the energy loss cost into the capacitor investment cost, the total cost can be reported in the table, and it is used as the most important comparison criteria for concluding the best performance method. As a result, the proposed method can reach smaller total cost than TLA and DSOA by $\$ 847.14$ and $\$ 3,786.46$, and it can reach the improvement level up to $0.87 \%$ and $3.77 \%$ as compared to TLA and DSOA. For the study case, TLA and the proposed method were run by setting $N_{\text {Po }}$ and $N_{\text {Iter }}$ to 50 and 100 and 30 and 100 . Clearly, the proposed method is very effective for the system. 
TABLE 10: Comparison with other methods for case 4 of the 85-bus system.

\begin{tabular}{|c|c|c|c|c|c|c|c|c|c|c|}
\hline Method & $N_{\text {Po }}$ & $N_{\text {Iter }}$ & Bus & Size & $\begin{array}{l}\text { Total capacity } \\
\text { (kVAr) }\end{array}$ & Min. loss (kW) & Mean loss (kW) & Max. loss (kW) & STD & $\mathrm{CT}(\mathrm{s})$ \\
\hline \multirow[t]{2}{*}{$\begin{array}{l}\text { Base network } \\
(\mathrm{Nc}=0)\end{array}$} & - & - & - & - & - & 316.12 & - & - & - & - \\
\hline & & & $\begin{array}{c}4 \\
7 \\
9 \\
21 \\
26 \\
30\end{array}$ & $\begin{array}{c}300 \\
150 \\
300 \\
150 \\
150 \\
0\end{array}$ & & & & & & \\
\hline \multirow[t]{13}{*}{ TLA [34] } & 50 & 100 & $\begin{array}{l}31 \\
45\end{array}$ & $\begin{array}{l}300 \\
150\end{array}$ & 2700 & 143.18 & 143.29 & 143.65 & 0.02209 & 18.38 \\
\hline & & & 49 & 150 & & & & & & \\
\hline & & & 55 & 150 & & & & & & \\
\hline & & & 61 & 300 & & & & & & \\
\hline & & & 68 & 300 & & & & & & \\
\hline & & & 83 & 150 & & & & & & \\
\hline & & & 85 & 150 & & & & & & \\
\hline & & & 4 & 293.00 & & & & & & \\
\hline & & & 8 & 289.64 & & & & & & \\
\hline & & & 12 & 385.25 & & & & & & \\
\hline & & & 20 & 237.23 & & & & & & \\
\hline & & & 28 & 165.22 & & & & & & \\
\hline & & & 34 & 165.06 & & & & & & \\
\hline \multirow{8}{*}{ MSFS } & 20 & 75 & 37 & 111.39 & 2723.7 & 142.4999 & 143.4605 & 145.2225 & 0.5573 & 15.72 \\
\hline & 20 & IJ & 40 & 212.05 & 2725.1 & 142.495 & 170.7000 & 170.228 & & \\
\hline & & & 46 & 34.25 & & & & & & \\
\hline & & & 52 & 212.13 & & & & & & \\
\hline & & & 61 & 142.53 & & & & & & \\
\hline & & & 65 & 183.18 & & & & & & \\
\hline & & & 70 & 133.77 & & & & & & \\
\hline & & & 73 & 158.99 & & & & & & \\
\hline
\end{tabular}

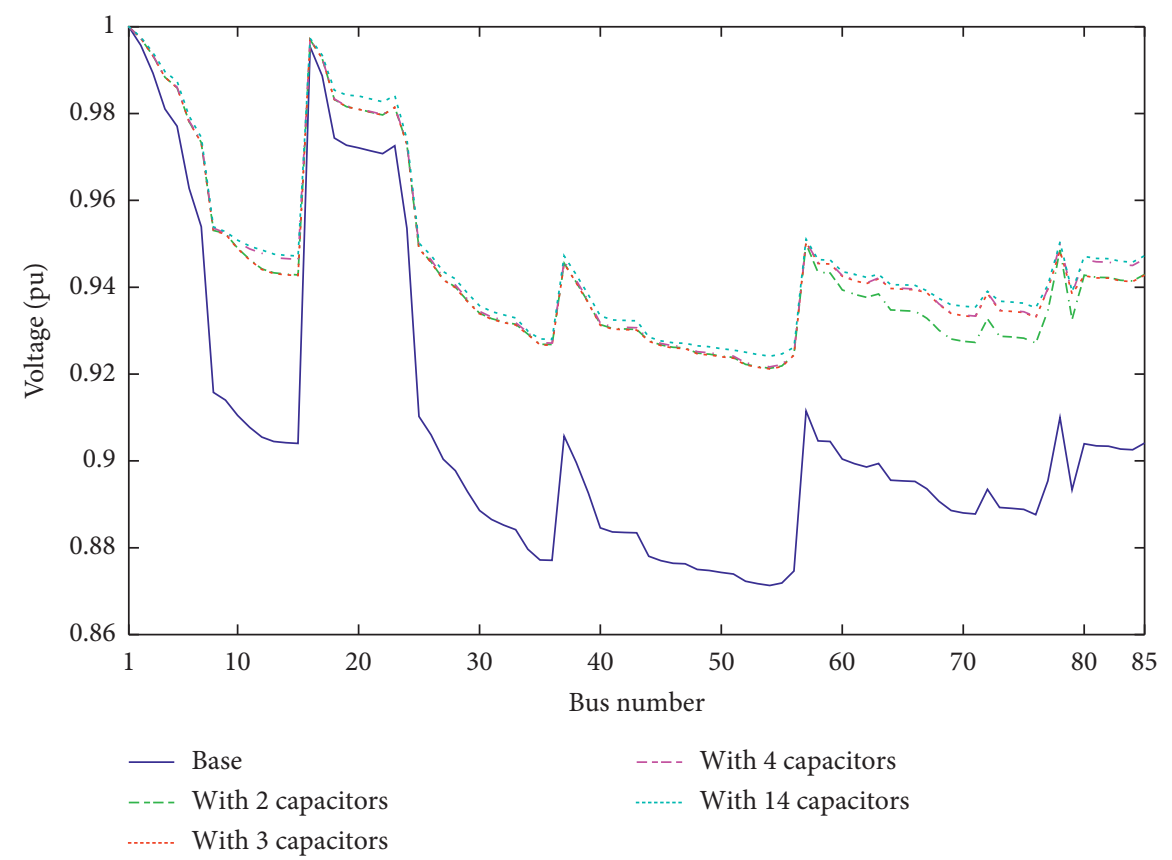

FIGURE 31: Voltage profile of 85-bus distribution system for different cases of capacitor placement. 
TABLE 11: Comparisons of result obtained by SFS methods for the 69-bus system with the objective function of total cost.

\begin{tabular}{lcccc}
\hline Method & SFS & MSFS1 & MSFS2 & MSFS \\
\hline Min. TC (\$) & 94181.33 & 94176.27 & 94157.14 & 94155.43 \\
Mean TC (\$) & 95859.22 & 95212.78 & 95491.23 & 95738.45 \\
Max. TC (\$) & 101285.85 & 100371.53 & 98667.72 & 101687.05 \\
STD & 1568.48 & 1209.47 & 1220.49 & 1723.38 \\
\hline
\end{tabular}

TABLE 12: Comparisons of result obtained by MSFS and other methods for the 69-bus system with the objective function of total cost.

\begin{tabular}{|c|c|c|c|c|c|c|c|c|c|c|c|c|}
\hline Method & \multicolumn{4}{|c|}{ TLA [34] } & \multicolumn{4}{|c|}{ DSOA [46] } & \multicolumn{4}{|c|}{ MSFS } \\
\hline$\overline{N_{\text {Po }}}$ & \multicolumn{4}{|c|}{50} & \multicolumn{4}{|c|}{-} & \multicolumn{4}{|c|}{10} \\
\hline$N_{\text {Iter }}$ & \multicolumn{4}{|c|}{100} & \multicolumn{4}{|c|}{-} & \multicolumn{4}{|c|}{30} \\
\hline Total cost $(\$)$ & \multicolumn{4}{|c|}{$95,316.38$} & \multicolumn{4}{|c|}{$99,912.60$} & \multicolumn{4}{|c|}{$94,155.43$} \\
\hline Energy loss cost $(\$)$ & \multicolumn{4}{|c|}{$89,016.38$} & \multicolumn{4}{|c|}{$89,112.60$} & \multicolumn{4}{|c|}{$87,855.44$} \\
\hline Capacitor investment cost $(\$)$ & \multicolumn{4}{|c|}{6300} & \multicolumn{4}{|c|}{10,800} & \multicolumn{4}{|c|}{6300} \\
\hline Total compensation capacity (kVAr) & \multicolumn{4}{|c|}{2100} & \multicolumn{4}{|c|}{3600} & \multicolumn{4}{|c|}{2100} \\
\hline Load status & & LL & NL & PL & & LL & NL & PL & & LL & NL & PL \\
\hline Power loss (kW) & & 34.43 & 146.8 & 417.28 & & 35.52 & 147 & 427.3 & & 34.228 & 145.87 & 419.016 \\
\hline \multirow{4}{*}{$\begin{array}{l}\text { Capacitor (location and compensation size of each } \\
\text { capacitor, kVAr) }\end{array}$} & Bus & & & & Bus & & & & Bus & & & \\
\hline & 22 & 150 & 300 & 300 & 15 & 300 & 450 & 900 & 68 & 150 & 150 & 150 \\
\hline & 61 & 450 & 1050 & 1050 & 60 & 300 & 450 & 900 & 61 & 600 & 1200 & 1650 \\
\hline & 62 & 150 & 300 & 750 & 61 & 450 & 900 & 1800 & 19 & 150 & 300 & 300 \\
\hline
\end{tabular}

LL: light load; NL: normal load; PL: peak load.

TABLE 13: Comparisons of result obtained by SFS methods for the 85-bus system with the objective function of total cost.

\begin{tabular}{lcccc}
\hline Method & SFS & MSFS1 & MSFS2 & MSFS \\
\hline Min. TC (\$) & 99035.78 & 97344.96 & 96956.99 & 96749.54 \\
Mean TC (\$) & 101444.3 & 97983.76 & 98290.09 & 97868.95 \\
Max. TC (\$) & 104181.6 & 99634.11 & 100138.2 & 101316.3 \\
STD & 1146.301 & 483.5635 & 738.4249 & 838.2434 \\
\hline
\end{tabular}

TABLE 14: Comparisons of result obtained by MSFS and other methods for the 69-bus system with the objective function of total cost.

\begin{tabular}{|c|c|c|c|c|c|c|c|c|c|c|c|c|}
\hline Method & \multicolumn{4}{|c|}{ TLA [34] } & \multicolumn{4}{|c|}{ DSOA [46] } & \multicolumn{4}{|c|}{ MSFS } \\
\hline$N_{\text {Po }}$ & \multicolumn{4}{|c|}{50} & \multicolumn{4}{|c|}{-} & \multicolumn{4}{|c|}{30} \\
\hline$N_{\text {Iter }}$ & \multicolumn{4}{|c|}{100} & \multicolumn{4}{|c|}{-} & \multicolumn{4}{|c|}{100} \\
\hline Total cost $(\$)$ & \multicolumn{4}{|c|}{$97,596.679$} & \multicolumn{4}{|c|}{100,536} & \multicolumn{4}{|c|}{$96,749.544$} \\
\hline Energy loss cost $(\$)$ & \multicolumn{4}{|c|}{$86,346.679$} & \multicolumn{4}{|c|}{86,586} & \multicolumn{4}{|c|}{$87,299.544$} \\
\hline Capacitor investment cost $(\$)$ & \multicolumn{4}{|c|}{11,250} & \multicolumn{4}{|c|}{13,950} & \multicolumn{4}{|c|}{9450} \\
\hline Total compensation capacity (kVAr) & \multicolumn{3}{|r|}{3750} & & \multicolumn{4}{|c|}{5650} & \multicolumn{4}{|c|}{3150} \\
\hline Load status & & LL & NL & PL & & LL & NL & PL & & LL & NL & PL \\
\hline \multirow[t]{7}{*}{ Power loss $(\mathrm{kW})$} & & 34.11 & 143.2493 & 411.6 & & 34.76 & 144.01 & 410.69 & & 34.943 & 144.871 & 415.389 \\
\hline & Bus & & & & Bus & & & & Bus & & & \\
\hline & 15 & 0 & 150 & 150 & 6 & 0 & 150 & 150 & 13 & 150 & 300 & 300 \\
\hline & 23 & 150 & 300 & 0 & 8 & 0 & 150 & 150 & 64 & 0 & 150 & 150 \\
\hline & 26 & 150 & 300 & 300 & 14 & 150 & 150 & 150 & 61 & 150 & 150 & 300 \\
\hline & 32 & 150 & 150 & 450 & 17 & 150 & 150 & 150 & 29 & 0 & 150 & 150 \\
\hline & 36 & 150 & 150 & 150 & 18 & 0 & 150 & 300 & 26 & 150 & 450 & 450 \\
\hline \multirow{9}{*}{$\begin{array}{l}\text { Capacitor (location and compensation size of } \\
\text { each capacitor, kVAr) }\end{array}$} & 38 & 0 & 150 & 150 & 20 & 0 & 150 & 300 & 73 & 0 & 150 & 150 \\
\hline & 45 & 0 & 150 & 150 & 26 & 0 & 150 & 150 & 70 & 150 & 150 & 300 \\
\hline & 52 & 0 & 150 & 300 & 30 & 150 & 300 & 450 & 35 & 150 & 0 & 150 \\
\hline & 57 & 150 & 300 & 300 & 36 & 300 & 450 & 900 & 2 & 0 & 0 & 0 \\
\hline & 61 & 0 & 150 & 150 & 57 & 150 & 150 & 300 & 50 & 0 & 150 & 150 \\
\hline & 64 & 150 & 300 & 450 & 61 & 0 & 150 & 300 & 53 & 0 & 150 & 300 \\
\hline & 73 & 150 & 150 & 300 & 66 & 150 & 150 & 300 & 32 & 0 & 150 & 150 \\
\hline & 82 & 0 & 150 & 450 & 69 & 150 & 300 & 600 & 45 & 150 & 150 & 150 \\
\hline & 84 & 150 & 0 & 0 & 80 & 0 & 150 & 450 & 10 & 300 & 150 & 450 \\
\hline
\end{tabular}




\section{Conclusion}

In this paper, the determination of the most appropriate site and size of capacitor banks has been implemented by the proposed MSFS method for reaching two single-objective functions which were the reduction of power loss and the reduction of total cost of energy loss and capacitor investment. The proposed method has been developed by performing the new diffusion mechanism and two new update mechanisms on the conventional SFS method. Three systems with 33,69 , and 85 buses have been solved by the proposed method, conventional SFS, and two other improved versions of SFS. The results from the proposed method, SFS, and other previous methods were compared for evaluation of performance. For the comparison with SFS, the advantages of the proposed method over SFS can be summarized as follows:

(1) For the first system with 33 buses, SFS and the proposed method could find the same best solution, but for the second study case of the system, SFS could not find the same best solution as the proposed method. The proposed method could find less loss than SFS by $0.263 \mathrm{~kW}$ corresponding to $0.002 \%$ of power loss.

(2) For the second system with 69 buses, the proposed method could find less power loss than SFS by $0.182 \mathrm{~kW}$ and $0.457 \mathrm{~kW}$, which were corresponding to $0.001 \%$ and $0.003 \%$ of loss from SFS. For the more complicated objective with the sum of energy loss cost and capacitor purchase cost, the proposed method could reduce the total cost to $\$ 25.9$ corresponding to $0.03 \%$ of cost from SFS.

(3) For the third system with 85 buses, the improvement of the proposed method over SFS was more significant for four study cases. For the first case with two capacitors, the loss reduction was $0.0294 \mathrm{~kW}$ corresponding to $0.0002 \%$, but for the following cases with higher number of capacitors, the reduction was higher. In fact, the loss reduction was, respectively, $1.8781,2.0274$, and $2.6125 \mathrm{~kW}$ corresponding to $0.0123 \%, 0.01345 \%$, and $0.018 \%$ for the cases with three, four, and fourteen capacitors. When considering the sum of energy loss cost and capacitor purchase cost, the proposed method could reach much higher performance than SFS by reducing the total cost to $\$ 2,286.24$, which was corresponding to $2.31 \%$.

These values of improvement level, which was represented by percent, were not high because SFS was also an effective method. Here, the performance improvement of the proposed method was about the ability to find the best solution and speed up the search process. The proposed method could find many solutions with the same quality as the best solution, whereas SFS could not reach the best solution even one time over fifty trial runs. When competing against other previous methods in solving the capacitor placement, the proposed method could find either the same good solutions or much better solutions. From the simulation results, the superiority of MSFS over other methods is as follows:

(1) For the 33-bus system, the power loss of the proposed method could be equal to that of a few methods but much smaller than many ones. The power loss reduction of the proposed method could be $0.07 \%$ and $3.98 \%$ of compared methods.

(2) For the 69-bus system, MSFS could achieve the improvement level over other ones from $3.7 \%$ to $7.3 \%$ for power loss and from $1.22 \%$ to $5.76 \%$ for the sum of energy loss cost and the capacitor purchase cost.

(3) For the largest system with 85 buses, MSFS could reach the improvement level from $0.92 \%$ to $6.98 \%$ for power loss and from $0.87 \%$ to $3.77 \%$ for the sum of energy loss cost and the capacitor purchase cost.

In terms of convergence speed, the proposed method was always much faster than other methods by setting smaller population size and smaller number of iterations. The parameter settings and simulation time can be summarized as follows:

(1) For the 33-bus system, the population size and iteration were set to 10 and 15 for the proposed method but 20 and 200 and 50 and 50 for other methods.

(2) For the 69-bus system, these parameters were 10 and 20 and 10 and 30 for the proposed method but 30 and 1000, 60 and 100, 50 and 100, and 2500 and 750 for other methods.

(3) For the largest system with 85 buses, the proposed method was run by setting 10 and 25, 20 and 30, 20 and 75 , and 30 and 100 for different cases, but they were much higher and equal to 50 and 100, 100 and 50 , and 20 and 200 for other methods.

(4) The simulation time of the proposed method was shorter than 1 second for the two first systems and from two to fifteen seconds for the last system, whereas the time of other methods was about ten seconds for the first two systems and about from ten to twenty seconds for some cases of the last system.

In summary, the proposed method could reach much better solutions than previous method, use less control parameter, and spend shorter simulation time. So, it can be concluded that the proposed method is an effective method for application of determining site and size of capacitors in distribution networks.

\section{Nomenclature}

Nbr: $\quad$ Number of branches of the considered distribution network

$\mathrm{Nb} \quad \quad \quad \quad$ Number of buses in the considered distribution network

Nc: $\quad$ Number of capacitor banks 


$\begin{array}{ll}N_{\mathrm{Df}}: & \text { Number of diffused times for each old solution } \\ N_{\mathrm{Po}}: & \text { Population size } \\ N_{\text {Iter }}: & \text { Number of computation iterations } \\ \text { Gbest: } & \text { The best solution of the current population } \\ X_{s}: & \text { The sth solution } \\ \varepsilon: & \text { A random number within } 0 \text { and } 1 \\ \mathrm{rd}_{s}: & \text { A random number within } 0 \text { and } 1 \text { produced for } \\ & \text { the sth solution } \\ X_{1}, X_{2}, X_{3}, & \text { Randomly selected solutions from the current } \\ X_{4}: & \text { population } \\ C_{\text {Iter }}: & \text { The current computation iteration } \\ \mathrm{QI}_{s}: & \text { Quality index of the sth solution } \\ R_{s}: & \text { Rank of the sth solution } \\ X_{s}^{\text {new }}: & \text { The sth new solution } \\ X^{\text {min }}, X^{\text {max }}: & \text { Minimum boundary and maximum boundary } \\ & \text { of control variables } \\ \mathrm{PF}_{1}, \mathrm{PF}_{2}: & \text { Penalty factors. }\end{array}$

\section{Data Availability}

Data of the 33- and 69-node distribution systems are taken from [38], and data of the 85-node distribution system are taken from [35].

\section{Conflicts of Interest}

The authors declare that they have no conflicts of interest.

\section{Acknowledgments}

This work belongs to the project grant no. T2020-43TĐ funded by Ho Chi Minh City University of Technology and Education, Vietnam.

\section{References}

[1] M. H. Haque, "Capacitor placement in radial distribution systems for loss reduction," IEE Proceedings-Generation, Transmission and Distribution, vol. 146, no. 5, pp. 501-505, 1999.

[2] D. Jakus, R. Čađenović, J. Vasilj, and P. Sarajčev, "Optimal reconfiguration of distribution networks using hybrid heuristic-genetic algorithm," Energies, vol. 13, no. 7, p. 1544, 2020.

[3] S. Guo, J. Lin, Y. Zhao, L. Wang, G. Wang, and G. Liu, "A reliability-based network reconfiguration model in distribution system with DGs and ESSs using mixed-integer programming," Energies, vol. 13, no. 5, p. 1219, 2020.

[4] H. M. Khodr, F. G. Olsina, P. M. D. O.-D. Jesus, and J. M. Yusta, "Maximum savings approach for location and sizing of capacitors in distribution systems," Electric Power Systems Research, vol. 78, no. 7, pp. 1192-1203, 2008.

[5] A. J. Wood, B. F. Wollenberg, and G. B. Sheblé, Power Generation, Operation, and Control, John Wiley \& Sons, Hoboken, NJ, USA, 2013.

[6] H. Manafi, N. Ghadimi, M. Ojaroudi et al., "Optimal placement of distributed generations in radial distribution systems using various PSO and DE algorithms," Elektronika ir Elektrotechnika, vol. 19, no. 10, pp. 53-57, 2013.

[7] W. Guan, Y. Tan, H. Zhang, and J. Song, "Distribution system feeder reconfiguration considering different model of DG sources," International Journal of Electrical Power \& Energy Systems, vol. 68, pp. 210-221, 2015.

[8] T. M. Khalil, A. V. Gorpinich, and G. M. Elbanna, "Combination of capacitor placement and reconfiguration for loss reduction in distribution systems using selective PSO," in Proceedings of the 22nd International Conference and Exhibition on Electricity Distribution (CIRED 2013), Stockholm, Sweden, June 2013.

[9] J. M. Home-Ortiz, R. Vargas, L. H. Macedo, and R. Romero, "Joint reconfiguration of feeders and allocation of capacitor banks in radial distribution systems considering voltage-dependent models," International Journal of Electrical Power \& Energy Systems, vol. 107, pp. 298-310, 2019.

[10] R. V. A. Monteiro, J. P. Bonaldo, R. F. da Silva, and A. S. Bretas, "Electric distribution network reconfiguration optimized for PV distributed generation and energy storage," Electric Power Systems Research, vol. 184, Article ID 106319, 2020.

[11] T. T. Tran, K. H. Truong, and D. N. Vo, "Stochastic fractal search algorithm for reconfiguration of distribution networks with distributed generations," Ain Shams Engineering Journal, vol. 11, no. 2, pp. 389-407, 2020.

[12] K. Muthukumar and S. Jayalalitha, "Optimal placement and sizing of distributed generators and shunt capacitors for power loss minimization in radial distribution networks using hybrid heuristic search optimization technique," International Journal of Electrical Power \& Energy Systems, vol. 78, pp. 299-319, 2016.

[13] S. Das, D. Das, and A. Patra, "Operation of distribution network with optimal placement and sizing of dispatchable DGs and shunt capacitors," Renewable and Sustainable Energy Reviews, vol. 113, Article ID 109219, 2019.

[14] A. R. Abul'Wafa, "Optimal capacitor allocation in radial distribution systems for loss reduction: a two stage method," Electric Power Systems Research, vol. 95, pp. 168-174, 2013.

[15] R. Viral and D. K. Khatod, "An analytical approach for sizing and siting of DGs in balanced radial distribution networks for loss minimization," International Journal of Electrical Power \& Energy Systems, vol. 67, pp. 191-201, 2015.

[16] K. Prakash and M. Sydulu, "Particle swarm optimization based capacitor placement on radial distribution systems," in Proceedings of the 2007 IEEE Power Engineering Society General Meeting, pp. 1-5, Tampa, FL, USA, June 2007.

[17] M. D. Reddy and V. V. Reddy, "Capacitor placement using fuzzy and particle swarm optimization method for maximum annual savings," ARPN Journal of Engineering and Applied Sciences, vol. 3, no. 3, pp. 25-30, 2008.

[18] R. S. Bapu, C. Prakash, and S. M. Kannan, "Optimal capacitor allocation in 69-bus radial distribution system to improve annual cost savings for dynamic load," International Journal of Emerging Technology and Advanced Engineering, vol. 3, no. 3, 2013.

[19] C.-S. Lee, H. V. H. Ayala, and L. d. S. Coelho, "Capacitor placement of distribution systems using particle swarm optimization approaches," International Journal of Electrical Power \& Energy Systems, vol. 64, pp. 839-851, 2015.

[20] Y.-T. Hsiao, C.-H. Chen, and C.-C. Chien, "Optimal capacitor placement in distribution systems using a combination fuzzyGA method," International Journal of Electrical Power \& Energy Systems, vol. 26, no. 7, pp. 501-508, 2004.

[21] K. R. Murthy, M. R. Raju, and G. G. Rao, "Comparison between conventional, GA and PSO with respect to optimal capacitor placement in agricultural distribution system," in Proceedings of the 2010 Annual IEEE India Conference (INDICON), pp. 1-4, Kolkata, India, December 2010. 
[22] S. Lohia, O. P. Mahela, and S. R. Ola, "Optimal capacitor placement in distribution system using genetic algorithm," in Proceedings of the 2016 IEEE 7th Power India International Conference (PIICON), pp. 1-6, Bikaner, Rajasthan, India, November 2016.

[23] M. Mahdavian, M. H. Kafi, A. Movahedi, and M. Janghorbani, "Improve performance in electrical power distribution system by optimal capacitor placement using genetic algorithm," in Proceedings of the 2017 14th International Conference on Electrical Engineering/Electronics, Computer, Telecommunications and Information Technology (ECTI-CON), pp. 749752, Phuket, Thailand, 2017.

[24] M. Dlfanti, G. P. Granelli, and P. Maranninio, "Optimal capacitor placement using deterministic and genetic algorithm," IEEE Transactions on Power Systems, vol. 15, no. 3, pp. 1041-1046, 2000.

[25] M. D. Reddy, "Optimal capacitor placement using fuzzy and real coded genetic algorithm for maximum savings," Journal of Theoretical and Applied Information Technology, vol. 4, no. 3, pp. 219-226, 2008.

[26] D. Das, "Optimal placement of capacitors in radial distribution system using a Fuzzy-GA method," International Journal of Electrical Power \& Energy Systems, vol. 30, no. 6-7, pp. 361-367, 2008.

[27] S. Nojavan, M. Jalali, and K. Zare, "Optimal allocation of capacitors in radial/mesh distribution systems using mixed integer nonlinear programming approach," Electric Power Systems Research, vol. 107, pp. 119-124, 2014.

[28] Y. M. Shuaib, M. S. Kalavathi, and C. C. A. Rajan, "Optimal capacitor placement in radial distribution system using gravitational search algorithm," International Journal of Electrical Power \& Energy Systems, vol. 64, pp. 384-397, 2015.

[29] A. Rajendran and K. Narayanan, "Optimal multiple installation of DG and capacitor for energy loss reduction and loadability enhancement in the radial distribution network using the hybrid WIPSO-GSA algorithm," International Journal of Ambient Energy, vol. 41, no. 2, pp. 129-141, 2020.

[30] M. I. A. M. Kowsalya, "Optimal Distributed Generation and capacitor placement in power distribution networks for power loss minimization," in Proceedings of the 2014 International Conference on Advances in Electrical Engineering (ICAEE), pp. 1-6, Vellore, India, January 2014.

[31] K. R. Devabalaji, K. Ravi, and D. P. Kothari, "Optimal location and sizing of capacitor placement in radial distribution system using bacterial foraging optimization algorithm," International Journal of Electrical Power \& Energy Systems, vol. 71, pp. 383-390, 2015.

[32] A. Y. Abdelaziz, E. S. Ali, and S. M. Abd Elazim, "Flower pollination algorithm and loss sensitivity factors for optimal sizing and placement of capacitors in radial distribution systems," International Journal of Electrical Power \& Energy Systems, vol. 78, pp. 207-214, 2016.

[33] V. Tamilselvan, T. Jayabarathi, T. Raghunathan, and X.-S. Yang, "Optimal capacitor placement in radial distribution systems using flower pollination algorithm," Alexandria Engineering Journal, vol. 57, no. 4, pp. 2775-2786, 2018.

[34] S. Sultana and P. K. Roy, "Optimal capacitor placement in radial distribution systems using teaching learning based optimization," International Journal of Electrical Power \& Energy Systems, vol. 54, pp. 387-398, 2014.

[35] D. B. Prakash and C. Lakshminarayana, "Optimal siting of capacitors in radial distribution network using whale optimization algorithm," Alexandria Engineering Journal, vol. 56, no. 4, pp. 499-509, 2017.
[36] E. S. Ali, S. M. Abd Elazim, and A. Y. Abdelaziz, "Improved harmony algorithm and power loss index for optimal locations and sizing of capacitors in radial distribution systems," International Journal of Electrical Power \& Energy Systems, vol. 80, pp. 252-263, 2016.

[37] K. R. Devabalaji, T. Yuvaraj, and K. Ravi, "An efficient method for solving the optimal sitting and sizing problem of capacitor banks based on cuckoo search algorithm," Ain Shams Engineering Journal, vol. 9, no. 4, pp. 589-597, 2018.

[38] A. Khodabakhshian and M. H. Andishgar, "Simultaneous placement and sizing of DGs and shunt capacitors in distribution systems by using IMDE algorithm," International Journal of Electrical Power \& Energy Systems, vol. 82, pp. 599-607, 2016.

[39] E. Mohamed, A.-A. A. Mohamed, and Y. Mitani, "MSA for optimal reconfiguration and capacitor allocation in radialring distribution networks," International Journal of Interactive Multimedia and Artificial Intelligence, vol. 5, no. 1, pp. 107-122, 2018.

[40] A. A. El-Ela, A. M. Kinawy, M. T. Mouwafi et al., "Optimal sitting and sizing of capacitors for voltage enhancement of distribution systems," in Proceedings of the 2015 50th International Universities Power Engineering Conference (UPEC), pp. 1-6, Stroke-on-Trent, UK, September 2015.

[41] A. Hamouda, N. Lakehal, and K. Zehar, "Heuristic method for reactive energy management in distribution feeders," Energy Conversion and Management, vol. 51, no. 3, pp. 518-523, 2010.

[42] A. Bayat and A. Bagheri, "Optimal active and reactive power allocation in distribution networks using a novel heuristic approach," Applied Energy, vol. 233-234, pp. 71-85, 2019.

[43] D. Q. Hung, N. Mithulananthan, and R. C. Bansal, "A combined practical approach for distribution system loss reduction," International Journal of Ambient Energy, vol. 36, no. 3, pp. 123-131, 2015.

[44] S. Kamel, A. Amin, A. Selim et al., "Optimal placement of DG and capacitor in radial distribution systems considering load variation," in Proceedings of the 2019 International Conference on Computer, Control, Electrical, and Electronics Engineering (ICCCEEE), pp. 1-6, Khartoum, Sudan, 2019.

[45] S. Kansal, V. Kumar, and B. Tyagi, "Hybrid approach for optimal placement of multiple DGs of multiple types in distribution networks," International Journal of Electrical Power \& Energy Systems, vol. 75, pp. 226-235, 2016.

[46] M. R. Raju, K. R. Murthy, and K. Ravindra, "Direct search algorithm for capacitive compensation in radial distribution systems," International Journal of Electrical Power \& Energy Systems, vol. 42, no. 1, pp. 24-30, 2012.

[47] J. Vuletić and M. Todorovski, "Optimal capacitor placement in distorted distribution networks with different load models using penalty free genetic algorithm," International Journal of Electrical Power \& Energy Systems, vol. 78, pp. 174-182, 2016.

[48] I. P. Abril, "Algorithm of inclusion and interchange of variables for capacitors placement," Electric Power Systems Research, vol. 148, pp. 117-126, 2017.

[49] S. Kola Sampangi and J. Thangavelu, "Optimal capacitor allocation in distribution networks for minimization of power loss and overall cost using water cycle algorithm and grey wolf optimizer," International Transactions on Electrical Energy Systems, vol. 30, no. 5, 2020.

[50] Z. Zandi, E. Afjei, and M. Sedighizadeh, "Reactive power dispatch using big bang-big crunch optimization algorithm for voltage stability enhancement," in Proceedings of the 2012 IEEE International Conference on Power and Energy (PECon), pp. 239-244, Kota Kinabalu, Malaysia, December 2012. 
[51] M. Sedighizadeh, H. Faramarzi, M. M. Mahmoodi, and M. Sarvi, "Hybrid approach to FACTS devices allocation using multi-objective function with NSPSO and NSGA-II algorithms in Fuzzy framework," International Journal of Electrical Power \& Energy Systems, vol. 62, pp. 586-598, 2014.

[52] H. Salimi, "Stochastic fractal search: a powerful metaheuristic algorithm," Knowledge-Based Systems, vol. 75, pp. 1-18, 2015.

[53] T. P. Nguyen and D. N. Vo, "A novel stochastic fractal search algorithm for optimal allocation of distributed generators in radial distribution systems," Applied Soft Computing, vol. 70, pp. 773-796, 2018.

[54] M. I. Alomoush and Z. B. Oweis, "Environmental-economic dispatch using stochastic fractal search algorithm," International Transactions on Electrical Energy Systems, vol. 28, no. 5, Article ID e2530, 2018.

[55] E. Çelik, "Incorporation of stochastic fractal search algorithm into efficient design of PID controller for an automatic voltage regulator system," Neural Computing and Applications, vol. 30, no. 6, pp. 1991-2002, 2018.

[56] L. H. Pham, M. Q. Duong, V.-D. Phan, T. T. Nguyen, and H.-N. Nguyen, "A high-performance stochastic fractal search algorithm for optimal generation dispatch problem," Energies, vol. 12, no. 9, p. 1796, 2019.

[57] J. Lin and Z.-J. Wang, "Multi-area economic dispatch using an improved stochastic fractal search algorithm," Energy, vol. 166 , pp. 47-58, 2019.

[58] T. T. Nguyen, D. N. Vo, H. V. Tran et al., "Optimal dispatch of reactive power using modified stochastic fractal search algorithm," Complexity, vol. 2019, Article ID 4670820, 2019.

[59] X. Chen, H. Yue, and K. Yu, "Perturbed stochastic fractal search for solar PV parameter estimation," Energy, vol. 189, Article ID 116247, 2019.

[60] T. T. Nguyen, T. T. Duong, M. Q. Duong, and A. T. Doan, "Optimal operation of transmission power networks by using improved stochastic fractal search algorithm," Neural Computing and Applications, vol. 32, pp. 9129-9164, 2020.

[61] E. Çelik, "Improved stochastic fractal search algorithm and modified cost function for automatic generation control of interconnected electric power systems," Engineering Applications of Artificial Intelligence, vol. 88, Article ID 103407, 2020.

[62] T. T. Nguyen, B. H. Dinh, T. D. Pham, and T. T. Nguyen, "Active power loss reduction for radial distribution systems by placing capacitors and PV systems with geography location constraints," Sustainability, vol. 12, no. 18, p. 7806, 2020.

[63] T. T. Nguyen, "A high performance social spider optimization algorithm for optimal power flow solution with single objective optimization," Energy, vol. 171, pp. 218-240, 2019.

[64] J.-H. Teng and C.-Y. Chang, "Backward/forward sweep-based harmonic analysis method for distribution systems," IEEE Transactions on Power Delivery, vol. 22, no. 3, pp. 1665-1672, 2007. 